\title{
ELEMENTARY PARTICLE PHYSICS
} AT THE UNIVERSITY OF FLORIDA

\section{ANNUAL PROGRESS REPORT DOE GRANT DE-FG05-86ER40272}

University of Florida

Gainesville, Florida 32611

December 1, 1991
Task A: Theoretical Elementary Particle Physics

Task B: Experimental Elementary Particle Physics Task C: Axion Project

Task E: SSC Detector Development

Task S: Computer Acquisition

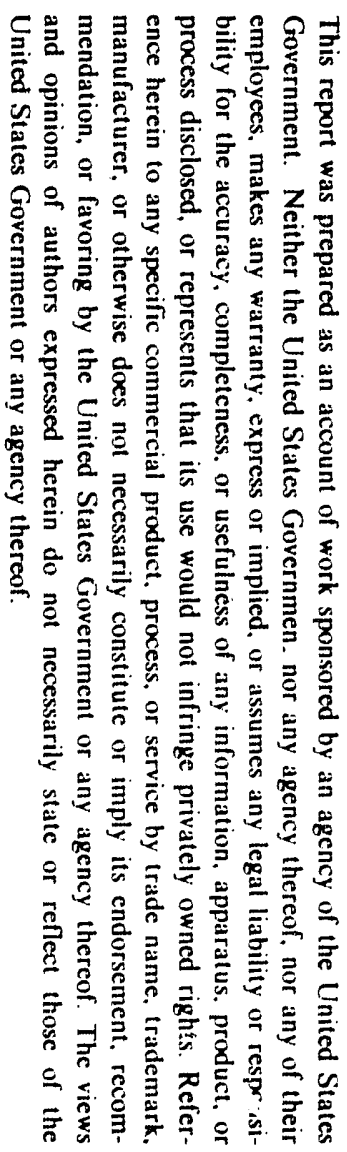

Grant Coordinator: R. D. Field

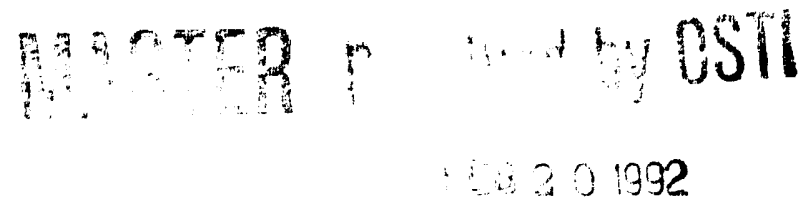


TASK A

RESEARCH IN THEORETICAL ELEMENTARY PARTICLE PHYSICS AT THE UNIVERSITY OF FLORIDA

\begin{abstract}
ANNUAL PROGIRESS REPORT
DOE GRANT DE-FG05-86ER40272
\end{abstract}

University of Florida

Gainesville, Florida 32611

December 1, 1991

Principal Investigators:

Richard D. Field

Pierre M. Ramond

Pierre Sikivie

Charles B. Thorn 


\title{
ANNUAL PROGRESS REPORT \\ TASK A
}

Theoretical Elementary Particle Research at the

University of Florida

\author{
Principal Investigators: \\ Richard D. Field \\ Pierre M. Ramond \\ Pierre Sikivie \\ Charles B. Thorn
}

\begin{abstract}
This is a progress report of the theoretical particle theory group at the University of Florida under DoE Grant DE-FG05-86ER40272. At present our group consists of four Full Professors (Field, Ramond, Thorn, Sikivie) and two Assistant Professors (Qiu, Woodard). In addition, we have five postdoctoral research associates and ten graduate students. The research of our group covers a broad range of topics in theoretical high energy physics with balance between theory and phenomenology. Included in this report is a summary of the last several years of operation of the group and an outline of our current research program.
\end{abstract}

\section{OUTLINE}

I. Introduction

II. History of the Particle Theory Group

III. Institute for Fundamental Theory (IFT)

IV. Particle Theory Personnel
(a) Faculty
(b) Postdocs
(c) Graduate Students

V. Scientific Statements of the Group Members

VI. Activities of the Particle Theory Group

VII. University Support for Particle Theory

Appendix A. Group Publications 


\section{INTRODUCTION}

This progress report on theoretical elementary particle physics is presented by the Particle Theory Group at the University of Florida.

In its short ten years of existence, our group has firmly established itself through solid research and educational contributions in theoretical physics. Its history appears in section II.

The Particle Theory group is part of the Institute for Fundamental Theory (IFT), an institute solely funded by the University. Its purpose is to enhance interdisciplinary interactions between different areas of theoretical physics and mathematics. Further details are to be found in section III.

At present our group consists of four Full Professors, R. D. Field, P. Ramond, C. Thorn, and P. Sikivie anid two Assistant Professors, Z. Qiu and R. Woodard. In addition, we have five postdortoral research associates, and ten graduate students. We have produced one $\mathrm{Ph} . \mathrm{D}$. eac's of the last five years. More details are to be found in section IV.

The scientific activities of our group are rich and varied, ranging from theoretical theory all the way to numerical phenomenology. At one end of the spectrum, the baffling conceptual theoretical problem of formulating a consistent quantum theory of gravity is one aspect of the research conducted here. The extraordinarily rich string theory as the only known consistent theory of quantum gravity provides a theoretical laboratory in which this difficult subject can be studied. Further, string theory offers new directions of study and links between hitherto unrelated fields of study. This is reflected by the research conducted here in statistical mechanics and integrable systems. Although many believe that string theory will link the experimentally remote Planck world with the accessible world of particle accelerators and other detectors, this has not yet been demonstrated. The low energy world is at present in a bizarre theoretical state as it is marvelously described by the Standard Model. No deviations from this model have yet been found, but it is widely believed that it is not fundamental but rather the chiral shard of a more integrated structure, perhaps string theory. In the meantime, the question of what lies directly beyond the standard model is being reinvestigated by our group, providing signatures for the structures that lie just beyond the grasp of experimentalists. Although the main theoretical extensions have been formulated a decade ago, they must be reexamined in the light of the better quality of the available data. Astrophysics also provides strong hints about the shortcomings of the standard model: many astrophysical phenomena, such as high energy cosmic rays, galaxy formation, and perhaps solar neutrino's and dark matter, are yei unexplained by the standard model. There also, the quality of the data has shown spectacular improvement. Our group is active in this area, both on the theoretical side and in the devising of new types of detectors for dark matter. Finally, the important question of detectability of new physics in colliders is addressed by the research of our group. This can only happen if the new physics signatures can be extracted from the everpresent standard model background. It is crucial to know which series of cuts have to be made to minimize the background and expose the new phenomena we all hope will emerge at the new accelerators. This computer intensive study is conducted in contact with 
experimentalists. The research conducted by the group is presented through individual's statements in section V. The productivity of our high energy theory group is reflected in the list of publication to be found in Appendix A.

In view of the geographical isolation of our campus, it is essential to the scientific survival and thriving of our group that we maintain a vigorous seminar and visitor program. Equally important is the ability for the members of our group to travel and collaborate with colleagues at other high energy physics universities and laboratories. The activities of the theory group center around a weekly seminar by an outside speaker, and a weekly journal club with an internal speaker. Graduate students hold an internal seminar of their own. In addition, a small number of longer term visits to the group are made possible through the IFT. In section VI we provide a list of last year's visitors as well as the professional activities of the group members.

Since the last contract review, our group has shown improvement in several areas. One is through the hiring of two new Assistant Professors, another is through the quality of our postdocs, and the third is through the emergence of a pool of good graduate students.

While the DOE has been very responsive in providing for our new faculty and postdocs, it has not supported with the same zeal our efforts to fund these graduate students. While a rationale could be made for this paucity of funding in the formative stages of the group, we now have shown success in attracting high quality graduate students. In each of the last four years, we have awarded one Ph.D., and placed the students in research postdocs. More are expected to graduate in the coming years, and many are actively collaborating with members of the group. We feel that training students in theoretical physics is an important role which we hope to continue to play with adequate support. Each year we send students to TASI. 


\section{HISTORICAL SURVEY}

The high energy theory group at the University of Florida was created in 1980 with the arrival of T. Curtright, R. D. Field, P. Ramond, and C. Thorn. The following year they were successful in acquiring a DOE grant. T. Curtright received a DOE Junior Investigator award. They were joined in 1981 by P. Sikivie. At that time the group had two postdoctoral associates, one funded by DOE and the other by the Department of Physics. In 1987, in response to the formation of the IFT, the group was allowed to increase by two junior faculty. These positions were filled by Z. Qiu in 1988 and R. Woodard in 1989. T. Curtright left the group in 1986 to join the faculty at the University of Miami. Within the next several years, we expect to fill his vacated position. Over the last ten years we have grown from two postdocs to the present five (we are one down from last year). In addition, we have been producing graduate students since 1987, in a steady stream. Two members of our group have received Guggenheim fellowships.

The rise of our group is but one aspect of the growth at the University of Florida. Over the last ten years, the Department of Physics at the University of Florida has more than doubled in size. In addition to the growth of the high energy theory group four faculty were hired in the area of theoretical astrophysics (Ipser, Detweiler, Fry, Whiting), one in the area of mathematical physics (Klauder), and five in condensed matter theory (Hirschfeld, Levine, Muttalib, Obukhov, Stanton). Schrieffer will join our Department, jointly with Florida State, on January 1, 1992. We have fruitful interactions with these groups through the Institute for Fundamental Theory (IFT). It has been more difficult to attract experimental high energy physics to Florida. However, we have succeeded in hiring three faculty in high energy experimental physics (Walker, Avery, Yelton). The increase in size and quality of the science personnel in Florida was recognized recently when the NSF awarded the Magnet Lab to Florida State and the University of Florida.

From 1980 to 1990, the Department of Physics at the University of Florida hired, on the average, about three new faculty per year. The high energy theory group has succeeded because of the conscientious efforts of the faculty and postdoctoral associates and because of the financial support we have received from the Department of Physics, the University of Florida, and the Department of Energy. 


\section{INSTITUTE FOR FUNDAMENTAL THEORY}

The particle theory group has benefited substantially from the creation of the Institute for Fundamental Theory (IFT) at the University of Florida. The IFT is an interdisciplinary center involving high energy theory (HET), condensed matter theory (CMT), cosmology-astrophysics, and mathematics and receives operating funds from the University of Florida. The aim of the IFT is to provide the right intellectual environment for attracting the brightest among young mathematicians and theoretical physicists. Our group has benefited directly from the improved environment provided by IFT. In addition, the IFT has contributed funds for high energy theory visitors and provides the salary for one of our particle theory postdoctoral research associates. Furthermore, the creation of the IFT was responsible for our sixth and seventh faculty position in particle theory.

We believe that in the years to come young theorists will tend to thrive in an multidisciplinary environment which includes close interaction between mathematicians and physicists. There are several reasons for this belief. First of all in physics there has been in the past ten years a dramatic confluence between hitherto independent discipline: namely, cosmology-astrophysics, particle physics, and condensed matter physics.

The marriage between cosmology and HET is well documented, and needs no elaboration here. So far the links between HET and CMT have occurred along the lines of quantum statistical mechanics, quantum field thieory, critical phenomena, and there has also been dramatic overlap between CMT and cosmology via the study of the kinetics of phase transition. In our view the overlap between CMT and HET will increase still more along the lines of deriving specific mechanisms of symmetry breaking. In particular it is hoped that the techniques for symmetry breaking in string theory might prove useful to the solution of CMT problems. In addition with the advent of string theory there is a need for a rapport between gravitation and HET, in order to arrive at a geometrical formulation of string gravity.

Just as there has been a tightening of the bonds between different areas of theoretical physics due to the universality of the methodis used, their mathematical origins have motivated a historic rapprochement with pure rnathematics. Thus the rationales for the IFT.

The intellectual environment of the High Energy Theory group has been enhanced by the IFT, as it brings distinguished visitors in closely related disciplines. In the recent past the IFT has funded long term visits to the group by Witten, Coleman, Singer, Brink, Goddard, Hartle to name a few.

In addition, the IFT has an external advisory board; its members are E. Abrahams, D. Eardly, Y. Nambu, and I. Singer. We expect the Institute to grow over the next several years as the Department of Physics plans to hire new faculty in the area of high energy and condensed matter theory. 


\section{PARTICLE THEORY PERSONNEL}

\section{(a) Faculty}

The following is a list of the faculty members of the particle theory group, their status and the length of their association with the group.

Name
T. Curtright

Position

DOE Outstanding Junior Investigator

$(9 / 1 / 82-8 / 1 / 84)$

Assistant Professor

$(9 / 1 / 84-9 / 1 / 85)$

Associate Professor

(9/1/85-8/31/89)

R. D. Field

Z. Qiu

P. Ramond

P. Sikivie

C. Thorn

R. Woodard
Professor (9/1/80-present)

Assistant Professor (9/1/89-present)

Professor (9/1/80-present)

Assistant Professor

(9/1/81-9/1/84)

Associate Professor

(91/85-8/31/88)

Professor

(9/1/88-present)

Professor

(9/1/80-present)

Assistant Professor

(9/1/89-present)

\section{(b) Postdoctoral Fellows}

In 1982, our first two postdocs were E. Braaten, now just recently tenured at Northwestern, and $M$. Chase who went on to CERN and then into industry in the UK. From 1983 to 1985 , we were joined by Richard Holman who went to Fermilab and is now an Assistant professor at Carnegie-Mellon. Our second crop of postdocs included M. Doria who went on to Los Alamos and is now in Brazil in a research position; J. Mc Cabe who was a postdoc from 1984 to 1986 and went on to LAPP in Annecy; and V. Rodgers who was with us from 1985 to 1987 and then went on to Stony Brook and is now an Assistant Professor at Iowa State.

In 1986, D. Harari joined our group. He is now in Buenos Aires in an academic position. Phil Oh from Chicago was here in 1986 and left having spent a year with us. He is now in a teaching position in Korea. In 1987, we were joined by D. Zoller, J. Minahan, S. Yost and A. Polychronakos, who was the first recipient of the IFT fellowship allocated to our group. In 1988, we acquired Christian Preitschopf from SLAC. In 1989, M. Awada came on board.

The summer before last, J. Minahan left to another postdoc at UVA and A. Polychronakos went to Columbia University. The extraordinarily competitive job situation for 
non-Russian non-phenomenologists that year resulted in neither Yost nor Zoller being able to secure a postdoc position at another institution. We chose to keep Yost for another year and filled our three vacant positions by hiring S. Sin (who is the second recipient of the IFT fellowship), S. J. Rey from Santa Barbara, and S. Martin from Princeton.

Last summer C. Preitschopf accepted a position at Gothenburg in Sweden, S. J. Rey left for a position at Yale, and M. Awada took a faculty position at the University of Cincinnati. Furthermore, S. Yost went to the University of Tennessee. We filled three of the four positions by hiring Y. Wang (from Carnegie-Mellon), P. Griffin (from FNAL), and M. McGuigan (Institute for Advanced Study). The following is a list of the past and present postdoctoral research associates of the particle theory group at the University of Florida.

Name

E. Braaten

M. Chase

M. Sato

F. del Aguila

M. Doria

R. Holman

V. Rodgers

J. McCabe

P. Oh

D. Harari

J. Minahan

D. Zoller

S. Yost

A. Polychronakos

C. Preitschopf

M. Awada

S. Martin

S. Rey

S. Sin

Y. Wang

M. McGuigan

P. Griffin
Length of Stay

$(9 / 1 / 81-8 / 31 / 83)$

$(9 / 1 / 81-8 / 31 / 83)$

$(9 / 1 / 82-5 / 1 / 83)$

$(9 / 1 / 82-8 / 31 / 84)$

$(9 / 1 / 83-8 / 30 / 85)$

$7 / 1 / 83-6 / 30 / 85)$

$(9 / 1 / 85-8 / 30 / 87)$

$(9 / 1 / 85-8 / 30 / 87)$

$(9 / 1 / 86-7 / 31 / 87)$

(9/1/86-8/31/89)

$(9 / 1 / 87.8 / 31 / 90)$

$(9 / 1 / 87-8 / 31 / 90)$

$(9 / 1 / 87-8 / 31 / 91)$

IFT, $9 / 1 / 87-8 / 31 / 90)$

(9/1/88-9/30/91)

$(10 / 1 / 89-8 / 31 / 91$

(9/1/90-present)

(9/1/90-7/31/91)

(IFT,9/1/90-present)

(9/1/91-present)

(9/1/91-present)

(10/20/91-present)
Present Location

Northwestern University

Industry

Japan

University of Barcelona

Brazil

Carnegie-Mellon University

Iowa State University

LAPP

Korea

Buenos Aires, Argentina

University of Virginia

University of Florida

University of Tennessee

Columbia University

Goteburg, Sweden

University of Cincinnati

University of Florida

Yale University

University of Florida

University of Florida

University of Florida

University of Florida

\section{(c) Graduate Students}

As we mentioned in the introduction, since 1987 we have been able to train and place graduate students.

M. Ruiz-Altaba, our first graduate student to obtain his Ph.D. was a transfer from Columbia university. He was awarded the diploma in 1987 under the supervision of Ramond. He went on to a first postdoc at CERN and is now into a second postdoc at the University of Geneva.

D.K. Hong was our second Ph.D. receipent, also under Ramond's supervision, in 1988. He went on to a postdoc at the University of Rochester and has presently secured a teaching position in Korea. Our third Ph.D. receipent u'as $\mathrm{R}$. J. Viswanathan. He received his degree in 1989 under Ramond's supervision and is now finishing his postdoc at the ICTP in Trieste.

M.F. Chu, under the supervision of Thorn, was our fourth graduate student to get a Ph.D. degree. She obtained her diploma in 1990 and is now in her first postdoc at the DAMPT in Cambridge. 
C. Hagmann obtained his Ph.D. last year under the supervision of P. Sikivie in conjunction with the axion detector. He is now at Berkeley, into his first postdoc. In addition, this year T. McCarty was awarded the Ph.D. degree. He was a student of T. Curtright, with Ramond acting as a surrogate advisor. He plans to go into industry.

We have graduated seven students to date, with several more students in the last stages of their research. G. Kleppe received his Ph.D. in the spring of 1991 under Ramond's supervision and is now starting a postdoc position at V.P.I.

The following table shows our past and present graduate students together with their advisor and their current position or status.

Name

M. Ruiz-Altaba

D. Hong

R. Viswanathan

M. Chu

T. McCarty

G. Kleppe

E. Piard

B. Keszthelyi

B. Wright

H. Arason

D. Castaño

J. Kim

S. Mikaelian

S. Carbon

J. Rubio

M. Tayebnejad

Advisor
Ramond
Ramond
Ramond
Thorn
Ramond
Ramond
Ramond
Ramond
Ramond
Ramond
Ramond
Sikivie
Thorn
Thoorn
Woodard
Field

Current Status

Ph.D. 9/1/87, University of Geneve

Ph.D. 9/1/88, Korea

Ph.D. 9/1/89, ICTP in Trieste, Italy

Ph.D. $4 / 1 / 90$, DAMPT in Cambridge, U.K.

Ph.D. 8/3/90, unemployed

Ph.D. $8 / 91$, V.P.I.

Ph.D. expected in 1993

$\mathrm{Ph} . \mathrm{D}$. expected in 1993

Ph.D. expected in 1992

Ph.D. expected 93-94

Ph.D. expected 93-94

beginning research

beginning research

beginning research

beginning research

beginning research

Under our present funding profile, graduate students are supported by the department with teaching assistantships (TA) during the Academic Year. We support most of these students with DOE and IFT funds during the summer. In addition, whenever funds permit the IFT supports a student in the last stages of research (Kleppe last year, Castaño this year). 


\section{Scientific Statements of the Group Members}

\section{(a) R. D. Field}

Much of my current research is related to the SSC and the SDC collaboration. At present, I am the University of Florida SDC institutional representative and a member of the "forward calorimeter" and "physics" subgroups. I have attended collaboration meetings in January, March, and August and as a member of the "forward calorimeter" subgroup , I have been looking at "generic" questions concerning the nature of "jets" in proton-proton collisions at the SSC. Some of the work has been done in collaboration with Mike Barnett (head of the subgroup) and Andy White. There are many interesting things to study and lots of questions to be answered.

I have been examining "jet shapes" in the forward region in high-energy proton-proton collisions at the SSC center-of-mass energy of $40 \mathrm{TeV}$. There are two contrasting ways in which jets can be produced in the forward region. Suppose that the two protons collide with their center-of-mass at rest in the laboratory and that a jet with a transverse energy of $100 \mathrm{GeV}$ is produced at a center-of-mass scattering angle, $\theta_{c m}$, of $2^{\circ}$ as a result of a hard 2-to-2 parton-parton scattering. One way this can occur is for the parton-parton 2-6o-2 subprocess, $a+b \rightarrow c+d$, to have its center of mass at rest in the laboratory (i.e., $x_{a} \approx x_{b}$ ). In this case, the energy of the outgoing "parent" parton would have to be about $2,865 \mathrm{GeV}$ and the parton-parton center-of-mass energy is large $(\approx 5,730 \mathrm{GeV})$. The particles forming the outgoing jet would, on the average, be distributed within a "cone" centered along the jet direction. Cones containing, for example, $80 \%$ of the jet energy typically have a halfangle of about $30^{\circ}$ so that jets arising in this manner would distribute their fragments over the entire forward region, $\theta_{c m}<30^{\circ}$.

A more economical way to produce a jet with a transverse energy of $100 \mathrm{GeV}$ at an angle of $\theta_{c m}=2^{\circ}$ is to produce, in the parton-parton center-of-mass frame, a $90^{\circ} 2$-to- 2 hard scattering in which the outgoing parton has a transverse energy of $100 \mathrm{GeV}$, but then "boost" this frame along the direction of the incident proton (i.e., $x_{a}>>x_{b}$ ). Since parton-parton cross sections decrease rapidity with their center-of-mass energy forward jets are dominantly of this type. The shape of a "boosted" jet remains roughly invariant when plotted versus pseudorapidity. Momenta in the transverse direction are invariant under a Lorentz transformation so that the jet shape in the azimuthal angle $\phi$ are unaffected. Hence a jet which at $\theta_{c m}$ (jet) $=90^{\circ}$ would have its particles located within the angular widths $\Delta \theta_{c m}=\Delta \phi=28^{\circ}$ (i.e., 0.5 radians), when "boosted" in the forward direction to $\theta_{c m}$ (jet) $=2^{\circ}$ becomes a "disk" with an azimuthal width of $\Delta \phi=28^{\circ}$ and an angular width of $\Delta \theta_{c m}=1$ !

Jets in the forward region (i.e., $\eta($ jet $)>4$ ) arise from 2 -to- 2 subprocesses in which the parton-parton center of mass is "boosted" along the incident proton direction (i.e., along the $\hat{z}$-axis). Because of the Lorentz contraction "jets" no longer appear, on the average, as cones. The shape in the azimuthal angle $\phi$ remains roughly invariant, while the shape in $\theta_{c m}$ is compressed. If we were using the true rapidity rather than pseudorapidity, the size would remain invariant. Shapes in pseudorapidity remain roughly invariant except for slow particles where mass effects become important. This means that "jets" in the forward region look, in the laboratory, more like sections of a "disk". Here, "jets" are defined using the "theoretical" definition of a jet (i.e., all the particles resulting from the fragmentation of an initial outgoing parton).

Instead of projecting the jet size on the pseudorapidity or the azimuthal angle axis, one can describe the size of a jet in terms of the "radius", $R$, in $\eta$ - $\phi$ space. Namely,

$$
R=\sqrt{d_{\eta}^{2}+d_{\phi}^{2}}
$$


where $d_{\eta}=\mid \eta-\eta($ jet $) \mid$ and $d_{\phi}=\mid \phi-\phi($ jet $) \mid$ and where $d_{\phi}$ is measured in radians.. On the average, $80 \%$ of the multiplicity and transverse energy of a "jet" is contained within a "radius" $R$ of 1.29 and 0.45 , respectively. An "average jet" contains $80 \%$ of its transverse energy within a "radius" of about 0.5 (with about $40 \%$ of the jet multiplicity within this radius).

Averages do not tell the whole story, however, as there are large fluctuations in QCD. For example, about $14 \%$ of the "jets" have $80 \%$ of their transverse energy contained within a "radius" of 0.1 , while for $10 \%$ of the $90^{\circ}$ jets and $20 \%$ of the forward jets one needs a "radius" greater than 1.0 !

Experimentally electromagnetic energy (i.e., photons and electrons) and hadronic energy are detected somewhat differently. Typically, the electromagnetic energy is detected with better resolution and a faster signal. Because of this it is interesting to see what we can learn about jets from a knowledge of their electromagnetic component. In particular, how well does the electromagnetic component (i.e., photons and electrons) of a jet "track" the true position of the jet. For an average "jet", $E_{T}^{e m}($ jet $) / E_{T}($ jet $)=0.24$, however, there are large fluctuations from this mean.

Examining the percentage of "jets" for which the electromagnetic component is located a "distance", $d_{e m}$, in $\eta-\phi$ space away from the true jet position gives, on the average, a $d_{e m}$ of about 0.17 , where

$$
d_{e m}=\sqrt{(\eta(e m)-\eta(\text { jet }))^{2}+(\phi(e m)-\phi(\text { jet }))^{2}}
$$

and $\eta(\mathrm{em})$ and $\phi(\mathrm{em})$ are the positions of the electromagnetic component of the jet. Again, however, there are large fluctuations from the mean. For example, about $27 \%$ of the "jets" have $d_{e m}$ less than 0.05 and about $10 \%$ of the "jets" have $d_{e m}$ greater than 0.3 !

I would like to continue to participate in the SDC collaboration as a phenomenologist and a "simulator". The SDC collaboration gives me the opportunity of working closely with experimenters on topics of mutual interest. I have always done my best work in collaboration with experimenters. I am just getting started in the SDC collaboration, however, I feel that I can be a valuable member of the group. I have recently joined the "physics" subgroup within the SDC and I would like to begin to contribute to the effort.

Now that we understand what "forward jets" look like we must decide whether it is important to be able to detect these jets. In particular, what interesting or new physics can one study by analyzing the forward jets. It has been suggested that perhaps "tagging" forward jets would allow one to isolate certain processes such as $W W$ fusion in the same way that tagging forward electrons in $e^{+} e^{-}$annihilations isolates photon-photon collisions. It is unlikely that forward jet tagging will be useful in hadron-hadron collisions since the ordinary QCD background events have many forward jets due to initial state bremmstrahlung. However, this needs to be studied in more detail. Also, the characteristics of these bremmstrahlung jets in the forward region needs to be examined.

Missing transverse energy is an important signal for new physics and requiring large missing transverse energy is a well known way to cut down on the ordinary QCD background. I am looking into the importance of the forward region $(\eta>3)$ in determining the missing transverse energy. I would like to understand better the rapidity coverage and resolution necessary at the SSC. 


\section{(b) Z. Qiu}

My research in the past few years is essentially in the following two directions: two dimensional critic l phenomena and string theory. The common link in these two directions in my research is the study of two dimensional conformal field theory.

It has been established by $\mathrm{K}$. Wilson and others that critical phenomena are described by fixed points of field theories in the dimensions under study. Above four dimensions the mean field theory gives the exact description of critical phenomena and in one dimension the phase transition can only occur at zero temperature. Therefore understanding two and three dimensional critical phenomena is one outstanding problem in the theory of critical phenomena. The renormalization group approach based on perturbation theory is the standard way to approach this problem. Much of our understanding of critical phenomena comes from the renormalization group study. Nevertheless, because of the simplicity of the problem involved, one would like to find an exact treatment of these problems. Moreover, classification of the fixed points of field theories will also shine new light on our understanding of the structure of quantum field theory.

Part of my work in the last few years was to understa 1 the two dimensional critical phenomena. The works of my collaborators and myself in the past few years are crucial parts in our recent understanding of two dimensional critical phenomena. We now know that all simple two-dimensional critical phenomena are described by rational conformal field theories (RCFT). RCFT are determined by very few fundamental assumptions and there is a working procedure to calculate all relevant physical information. Some of my works in this direction are listed here. Working witk D. Friedan and S. Shenker we investigated the relationship between two dimensional conformal field theory, critical phenomena and string theory. Our work uncovered that critical exponents in the kinds of two dimensional critical systems most often encountered - genuine thermal phase transitions with spatial isotropy - are constrained to have certain rational values. This explained the occurrence of rational critical exponents in many known two-dimensional systems. We also extended our work to conformal field theory with extra symmetries, e.g. supersymmetry. A laboratory system had been identified that displays this exotic structure. It provides the first realization of a supersymmetric field theory in nature. Our study of superconformal symmetry also led to the completion of covariant quantization of superstring theory and a practical way of computing amplitudes in superstring theory. Working with D. Gepner, we investigated the role of modular invariance in two dimensional conformal field theory. The spectrum of conformal field theory is completely determined by modular invariance. We classified parafermion conformal field theories. Our work also proved a conjecture of Capelli, Itzykson and Zuber on the classification of discrete conformal field theories. In 1986, I found a realization of the $\mathrm{N}=2$ discrete superconformal field theories. Using this realization I classified all the discrete $\mathrm{N}=2$ superconformal field theories. This work is a crucial step which later led to works of many people on finding exact superstring compactifications, i.e. exact Calabi-Yau compactifications, and more recent works on the relationship between conformal field theory and catastrophe theory. Working with D. Kastor and E. Martinec, we realized that chiral algebra is the underlying organization principle of rational conformal field theories. This observation together with the examples point ou a new way to characterize all rational conformal field theories. Working with J. Distlex, we described a bosonization of $\mathrm{SU}(2)$ Wess-Zumino-Witten model and investigated the general question of bosonization of conformal field theories.

My interest in string theory arises from the fact that it is the only known theory which (apparently) consistently incorporates both quantum mechanics and general relativity. Two dimensional conformal field theory is a very powerful tool, in both practical calculations and general formulation, in studying string theory. One of the crucial questions in 
string theory is how nature selects one particular vacuum, our world, out of millions of solutions of string equation of motion. String field theory seems to be one promising way to study this non-perturbative aspect of string theory. Unfortunately the present formulation of string field theory cannot even describe all the solutions of the string equation of motion. In a recent work, Tye and I proposed a configuration space for string field theory. It is essentially the space of all conformal field theories, both unitary and non-unitary, of central charge $c=26$ ( 15 for superstring). The configuration space includes all the solutions of string equation of motion. Moreover our proposal also suggests a much larger symmetry underlying string theory. I intend to continue our work in this direction. We are now in the process of finding the kinetic term of string field theory in our formalism. We will next study the geometric structure of this configuration space. Hopefully, it will lead us to the completion of string field theory action. I also intend to attempt to apply these ideas towards a calculation of non-perturbative quantum effects while working on the formal aspect of string field theory.

In the last few months I have been working with M. Awada, a postdoc in our group, on problems in 2-d quantum gravity theories. We study the algebraic origin of the nonperturbative equations appearing in the matrix model study of 2-d rravity theories. These equations are known to contain all the information of the theories. Our work is reported in three manuscripts to be published in Phys. Lett. B.

In the next three years, I plan to concentrate my research activities around understandiag three dimensional critical phenomena, in particular the exact treatment of this problem. I firmly believe that research in this area will have far greater impacts on physics than those have been achioved in studying two dimensional critical phenomena. The first step in my study is to understand the implications of many recent exact results in topological field theory, non-critiral string theory and find the link, if it exists, between these problems. Some work in this direction is carried out in our work on two dimensional quantum gravity and supergravity (non-critical string theories).

\section{(c) P. Ramond}

In 1987, I spent much of my time writing additional chapters to appear in the second edition of my book on field theory. These were on finite temperature methods, gravity as a gauge theory, and anomalies. In the meantime my fascination with string field theory continued, but in spite of several attempts detailed in four publications with students $R$. Viswanathan and G. Kleppe, we only succeeded in rediscovering known facts in a different language.

In 1988, motivated by Witten's analysis of $2+1$ gravity as Cherns-Simons theory, in collaboration with students E. Piard and B. Khestezlyi, we studied the meaning of a duality constraint $I$ had formulated in 1981, in the spirit of Eguchi-Hanson Ansat $z$ in $3+1$ dimensions. We showed that in the Witten sense it corresponds to a pure gauge configuration, and evaluated the partition function around it. In the same paper we investigated a special type of Weyl theory in $2+1$ dimensions, describing gravity in interaction with a gauge potential through Weyl's original gauge invariance, and a non-canonical scalar field. Interestingly, we found that the gauge field acts as the velocity vector of a perfect fluid; furthermore it obeys the Chern-Simons self duality condition. The theory was solved in the static case, leading to a Liouville equation. In the further restriction of axial symmetry, a solution corresponds to that found by Godel. The paper was preprinted this spring and has been accepted for publication by JMP.

In the spring of 1989 , I realized that we were not teaching our students the Standard Model, and that as a result they were not versed in the techniques of perturbative quantum field theory. In view of the ongoing and pending exciting experiments at CERN, SLAC 
and FERMILAB, and solar neutrinos, I thought I should teach a course based on the Standard Model and its minimal extensions, such as neutrino masses, axion, extra Z's, supersymmetry,..., that is dealing with issues which might be resolved in the present or uext round of experiments. Last year I wrote extensive notes on some of these subjects, but found myself spending much more time on the Standard Model itself, with the result that I will teach the course this coming year, covering all the topics I left uncovered the first year. My present plan is to write a book based on these notes with the title of "Journeys beyond the Standard Model". In this purely educational process, I found several noteworthy points which had escaped earlier investigators. I used these hitherto unnoticed facts as laboratories for students and postdocs. This has worked very well. So far three papers have been written in this way. The first involves hypercharge assignments in the Standard Model, where it is pointed out that anomaly cancellation alone is sufficient to pinpoint the hypercharges and, upon electroweak breaking, the electric charges. Use is made of the mixed gravitational anomaly. This point, as we subsequently found, had been made earlier by Geng and Marshak, so we wrote it up as a comment to their paper; it appeared in PRD41,715(1990), in collaboration with J. Minahan and R. Warner.

A second paper was generated by examining the constraints put on the LEP-SLC width measurements to the existence of exotic anomaly free chiral fermion sets beyond the expected three families. The paper appeared in PRD..., in collaboration with J. Minahan, and a student, $B$. Wright.

Another paper originated while surveying the different ways in which one can obtain massive neutrinos in the Standard Model; it turns out that there is a rather natural way of introducing neutrino masses into the SM with invisible axion by just adding one charged Higgs scalar; this represents a very minimal extension. A paper detailing the model and its experimental signatures is being preprinted, in collaboration with two students $\mathrm{H}$. Arason and $B$. Wright; it will be submitted to PRD.

Another paper is at very preliminary stage; it is based on the following observation: the large value of the top quark mass creates a intra-family hierarchy between the bottom and top quarks. One way to account for this is to start with the SM with the Higgs coupling to the charge $2 / 3$ sector only. In this case, masses for the other charged particles will never develop due to chiral symmetry. But when we supersymmetrize this model, the Higgsino doublet has a chiral anomaly which must be cancelled; there are two ways to do this- in the first, one introduces a vector-like partner to the Higgsino, which has a scalar partner with the opposite hypercharge, and this is exactly the minimal supersymmetric standard model. However, the anomaly can be cancelled in another way, by inventing a whole family with the opposite hypercharge. We are exploring this non-minimal possibility; early investigations indicate that in such a model $\mathrm{R}$-parity is violated, which is extremely dangerous as well as exciting. It turns out that the model cannot work without introducing a neutral lepton in the model. Should the model succeed, it would offer an explanation for the difference between the top and bottom masses by saying that while the former is only forbidden by electroweak symmetry, the latter is forbidden by both electroweak symmetry and supersymmetry. This is the idea, and I plan to explore this model during the fall with one or more student and probably during the class as well.

In another unrelated subject I am investigating with student D. Castaño and Lars Briak. fractional powers of functional determinants. The idea, based on attempts to co$v / \Delta r i a n t l y$ quantize the Green-Schwarz action, makes use of the metaplectic representation if orthosymplectic groups which have fractional dimensions. We are interested in using this idea to evaluate the square root of the determinant of the covariant derivative in $2+1$ dimensions, where Weyl projections are not available. We are at present evaluating the determinant in perturbation theory, with the idea that it could map itself into a relativistic description of semions. 
In terms of future projects, I am interested in studying Alain Connes' idea of of applying his non-commutative geometry to the Standard Model. I intend to see if it can be applied to a GUT theory such as $S O(10)$ and $E_{6}$. I am quite intrigued by Belissard' application of these ideas to the fractional Hall effect. This represents to me a general cultural interest in these matters. I am also curious of the possible application to quasi-crystals.

Finally, I have been thinking about how one may formulate lattice models in more than two dimensions. In analogy with the equivalence principle relating the structure of the bundle to that of the manifold, I have tried to erect over lattices whith have an errorcorrecting code interpretation, systems of spin, to be interpreted as words. This program has not gone very far, but I keep thinking about it.

In the period of interest, I have graduated several Ph.D. students; they are: $M$. RuizAltaba (1987), D. K. Hong (1988), R. R. Viswanathan (1989).

I anticipiate the following graduations: G. Kleppe (1991), B. Wright (1992), B. Keszthelyi (1992), with other students in the wing, E. Piard, H. Arason, and D. Castaño.

UPDATE (OCTOBER 1991)

The paper with Arason and Wright has now appeared in PRD

Nothing has come of the paper on creating an intrafamily hierarchy for the quark masses. Most of my time was taken up last year in teaching the course on the Standard Model and beyond. I have written extensive new notes on dynamical supersymmetry breaking, and the super standard model: SSC, the Super Standard Construct.

My student, Diego Castaño, has finished the project started with Lars Brink and myself. He wrote a paper by himself which has been accepted for publication in Physics Letters B.

This spring, my students, under my supervision have undertaken a mammoth project on plotting all of the Standard Model's parameters up to the Planck scale. This work includes proper account of thresholds, two loop effects, and an algorithm for bringing the initial data to the same scale. As this is being written, a long paper is in the last stages of preparation; we call it the template. The idea is to use it as a template upon which new models are added. I am not an author on this paper, although I have guided them extensively. For several of them, this paper will be an important part of their thesis.

In late spring, we had to depart from our game plan which was first write the template, then add supersymmetry. The work of Amaldi et al. forced us to publish over the summer a paper on the Yukawa couplings in the minimal supersymmetric extension of the Standard Model. In it we use the SUSY unification scale to demand that the bottom and tau Yukawas be the same there. Then, after comparison with upsilon physics at $10 \mathrm{GeV}$, we infer from the running of the couplings bounds on the top quark masses. Remarkably, we find that the top quark mass comes out less than $200 \mathrm{GeV}$, in accordance with the $\rho$ parameter. This work has just been accepted for publication by Physics Review Letters.

Last week I just signed a contract with Addison-Wesley to write a book on Standard Model Extensions. I intend to devote a good portion of n:y time to the writing of the book which is based on the notes I have developed.

This year I graduated my fourth Ph. D. student at Univ. of Florida: Gary Kleppe. This coming year I expect to graduate one more for sure, Brian Wright, and possibly another. I am delighted by the progress of the students.

This year I am serving on HEPAP. So far I have attended only one meeting. I anticipate this service to be quite time-consuming in spurts, as well as expensive to the contract. 


\section{(d) P. Sikivie}

Last fall Chris Hagmann and I completed our study of the motion and radiation spectrum of global strings. Our work was motivated by the interest in evaluating the size of the axion cosmological energy density, and by the possibility that cosmic global strings exist in the universe today and reveal themselves by the conversion of their Nambu-Goldstone radiation to photons in astrophysical magnetic fields. Our computer simulations are in agreement with the conjecture proposed earlier, hy Diego Harari and myself, that global strings decay practically as fast as causality allows them to and that the shape of their radiation spectrum is $d E / d k \sim 1 / k$. In our paper, Chris Hagmann and I also gave theoretical arguments why this is the most plausible spectrum.

Jim Ipser and I wrote a paper on the phase space structure of cold dark matter halos. We were originally motivated by the question what information can be learned from a signal in a earth based dark matter detector. It has usually been thought (or implicitly assumed) that the spectrum of dark matter particles is a smooth bump, rather similar to a Maxwellian distribution, because the kinetic energies of the particles thermalize while the Galaxy forms. However, Jim Ipser and I showed that the thermalization of collisionless cold dark matter particles in a galaxy such as ours is far from complete after $10^{10}$ years. We predict that the energy-momentum spectrum is composed of peaks, of order 100 in number, some of which are very narrow.

San-Jin Sin, who is a post-doc in our Particle Theory Group, and I want to follow up on this by studying the effect of the sheet-like distribution of cold dark matter particles in phase space upon the motion of the stars. Our main result so far is that dynamical friction tends to lock some fraction of the stars onto the phase space sheets.

Jae-Wan Kim, who is my Ph.D. student, Jooyoo Hong, who is visiting us on a scholarship from the Korean Government, and I are studying the effect of small wiggles on cosmic strings. We have independently obtained a number of results which were already published, plus a number of new results. Among the latter are the effective equations of motion for a Nambu-Goto string with large amounts of smail wiggles on it. The equations treat the wiggles as a fluid attached to the string. We are presently applying the equations to a number of particular cases.

At the moment, most of my time and energy is spent on completing the review article on Axions for Physics Reports, which I started five or six years ago. I hope to finish it by December 1991. I am teaching an advanced topics course on Axions this fall. The course gives me many opportunities for refining my understanding of the subject.

\section{(e) C. B. Thorn}

I completed a major review of string field theory in Fall, 1988, which was published in Physics Reports in 1989. After that there were several unresolved issues in the application of string field theory to the superstring. Together with two postdocs, Scott Yost and Christian Preitschopf, we showed how these problems could be solved by setting up the theory in a different picture for the superghosts. This work is described in a long article that has appeared in Nuclear Physics (1990). Our work on string field theory was restricted to open string fields. Such theories predict closed strings as "bound states" of open strings. A major open question, which our work does not touch on and which I hope to turn to in the future, is the application of string field theory to purely closed string theories, including the heterotic string.

In the summer and fall of 1989 I worked with Pierre Sikivie on a completely different project to resolve some issues in QCD involving the phase of the chiral $\bar{q} q$ condensate. This phase cannot be determined by the mass splittings of the octet of pseudoscalar Goldstone 
bosons, but we clarified how it is fixed theoretically. A description of this work is published in Physics Letters.

During 1990 I worked on "subcritical string theory", the theory of strings in less than the critical space-time dimension. There had been an explosion of work on this subject for space-time dimension $D \leq 1$, for which large $N$ matrix models were applicable. I decided to return to the approach to subcritical strings using Liouville field theory. This approach, suggested first by Polyakov in 1981, was vigorously developed by Braaten, Curtright, and me in the early '80's (1982-1984). Lacking progress in calculating exact correlation functions in the quantum Liouville theory, I suggested taking a "phenomenological" approach by investigating the open string dual loop expansion in subcritical dimensions. It had previously been thought that such theories would be inconsistent with unitarity, but I showed that a sharper way to describe the situation is to interpret the "unitarity violating cut" in the one loop amplitudes as due to a continuous mass spectrum for the closed string. With this new interpretation, one can abstract information about subcritical string theory by studying the dual loop expansion in detail. With Christian Preitschopf, we developed an open string field theory for subcritical dimensions by constructing an action principle which generates the open string dual loop expansion. We hope these results will be useful in further understanding the matrix model approach for $D \leq 1$ as well as subcritical string theory for $1 \leq D \leq 25$. This work was published in a recent issue of Nuclear Physics B.

I spent spring semester 1991 on sabbatical at Cambridge University in England. My work there was devoted to trying to sharpen our formulation of string dynamics. One issue raised by recent activity in subcritical string theory is the fate of the graviton so prominent in th critical theory. Unfortunately the only really complete treatment of subcritical string theory, via matrix models, works only for $D \leq 1$, where the issue of gravitons does not arise. Nonetheless, partial understanding for the case $1 \leq D \leq 25$, indicates the absence of a massless spin 2 particle in the spectrum. The massless spin 2 particle for $D=26$ forces a gauge group at least as rich as general coordinate invariance. Normally we associate such a gauge group with massless gravitons, so what could be happening in $D<26$ ? The most. mundane possibility is that the apparently massive graviton suggested by the Liouville theory is an artifact due to expanding the theory about a wrong vacuum, analogous to expanding Einstein's theory with cosmological constant about flat space-time. It is well known that efforts to give the graviton a mass seem inevitably to lead to ghost or stability problems, but those conclusions might have loopholes. With Michael Green, we studied an example in string theory, viz. open string theory in tree approximation where there is no massless graviton. We constructed an interpolation between this theory and closed string theory (which has a graviton). We showed that the intermediate theories do have ghosts and clarified the manner in which consistency is regained in the two limits. This example, shows how the generic problems expected from giving the graviton a mass might conceivably disappear for a discrete value of the mass. This work was submitted for publication in Nuclear Physics.

Also during this period (Spring, 1991), I worked on developing the idea that relativistic strings are composite objects. This is motivated by the desire to bring string theory into the framework of ordinary quantum field theory, and also by the growing feeling that the apparatus of string field theory is overly cumbersome and perhaps not even internally consistent. If strings were bound states of ordinary field excitations, it would be wrong to assign string states a fundamental role in the formulation of the dynamics. I reworked the so called "fishnet" model of composite strings into an explicit postulate for an ordinary (unfortunately not Lorentz covariant) field theory on a D-1 dimensional manifold. This exercise shows that it is possible to get the rich structure of string theory from even less than a field theory in D dimensions, but it is hardly the final answer, lacking Lorentz covariance for one thing. A field theory underlying strings, if it exists, should have general coordinate 
invariance, but neither strings nor a graviton as input. As candidates for such theories I plan to investigate "topological gravity" theories and "conformal supergravity" theories. These are both examples of generally covariant field theories without input gravitons, and as such might have some light to shed on string theory. These ideas were presented in an invited talk to the Sakharov Conference in Moscow (May 1991) and will appear in the proceedings.

One of the driving motivations for thinking about subcritical string theory is the old hope that string theory might be a useful tool for describing quark confinement in QCD. While it is undoubtedly true that the 't Hooft's $1 / N_{c}$ expansion of QCD leads to some kind of a string theory (assuming quark confinement), I suspect that this "QCD string" is quite a different object than the subcritical string obtained from dual models. For one thing, the QCD string must be compatible with asymptotic freedom and contain hard point like structures. For another, QCD is not generally covariant and hence could never produce a graviton in a consistent approximation. In the next couple of years I plan to renew efforts I began in the late 70's to develop a working theory of such a QCD string. In that work I tried to combine light-cone ("light-front") quantization with the $1 / N$ expansion to formulate the dynamics of the QCD string. Unfortunately, I did not succeed in solving this dynamics. I hope that the time is now ripe to make further progress in this direction.

\section{(f) R. Woodard}

My basic interest is quantum gravity in the larger context of Lagrangian field theory and particle physics. The two major problems in this area concern extremely short and extremely long distance scales. The short distance - or "ultraviolet" - problem is that quantum corrections to Einstein's theory diverge in an uncontrolable way. The long distance - or "infrared" - problem is that Einstein's theory has an apparently free parameter called the "cosmological constant" whose value must be fine tuned to one part in $10^{120}$ in order to prevent the radius of the universe from either shrinking to near zero or else expanding exponentially.

My past year's work on the ultraviolet problem has been done in collaboration with Dr. Gary Kleppe, formerly a graduate student in our group and now a postdoc at Virginia Polytechnic Institute. Our effort has consisted of the elaboration of nonlocal regularization. This is a technique by which any divergent, local quantum field theory can be transformed into an ultraviolet finite, nonlocal one which has the same S-matrix as its local parent. The method can either be used as a regularization of existing theories or to generate candidate fundamental theories. It is this latter use which may be relevant to the ultraviolet problem of quantum gravity.

To provide a detailed check of nonlocalization as a regularization Gary and I used the method to renormalize $\phi^{4}$ theory in four dimensions and $\phi^{4}$ theory in six dimensions at one and two loops. We showed that the method functions properly despite its explicit introduction of nonlocality. In particular there was no problem with overlapping divergences. We also found a general way of inferring the nonlocally regulated result at any order from the dimensionally regulated one.

Any symmetry possessed by the local parent theory will appear in the nonlocalized theory but possibly in a distorted form. If a suitable functional measure factor can be found so that quantization preserves these symmetries then the nonlocalized theory is not only finite but also perturbatively unitary. The existence of such a measure has been proven for $Q E D$. This also implies the existence of a version of $Q E D$ which is free of vector and axial vector anomalies and which still manages to give the correct rate for $p i^{0} \rightarrow \gamma \gamma$. The measure factor for pure Yang-Mills has been constructed at lowest order but there is 
as yet no proof that it exists to all orders. An ansatz which we are now checking is that the relation which holds for scalar field theories between dimensional regularization and our method applies generally. If this is so then we have the measure factor in all cases, such as pure Yang-Mills and pure gravity, where dimensional regularization works.

Gary and I were able to prove that the nonlocal regularization of any theory represents a noncanoncial quantization of the local equations of motion. This means that the method is free of the extra solutions which typically occur when higher derivatives are added. It suggests that the key to successfully quantizing gravitation may not involve changing the field equations, as has always been suggested previously, but rather changing the method of quantization.

My past year's work on the infrared problem has been done in collaboration with Professor Nicholas Tsamis of the University of Crete. We noticed this summer that on dimensional grounds quantum loop effects ought to give an intense enhancement of Newton's law in the far infrared when the cosmological constant is nonzero. Explicit calculations in Einstein's theory confirm this expectation. Since the theory ought to be reliable in the far infrared these predictions are not affected by the still unresolved ultraviolet problem. The familiar process of particle production in an inflating universe provides widely separated sources of stress energy which would be attracted by the strengthened long range force. We believe that this effect is sufficient to halt the expansion of spacetime as though the cosmological constant had been zero to start with. We also speculate that the heavy dependence of this infrared effect upon the dimension of spacetime may offer an explanation for why there are only four approximately flat dimensions.

\section{(g) M. Awada}

This was my last year at the University of Florida. I have taken a position at the University of Cincinnati as a post doc with the agreement it will turn into a faculty position. My current research is directed towards incorporating modular geometry in quantum field theory and in Quantum Closed String Field Theory (QCSFT). I have proposed a completely new formula for the full quantum Hamiltonian to all loop orders in perturbation theory. The new expression is very different from the naive forms proposed previously in the literature. This Hamiltonian does not depend on any particular topology or genus of the Riemann surfaces involved. This is because the space over which we are integrating is the space of all moduli space $\mathrm{R}$. This new modular QCSFT give a finite ultraviolet theory to all loop orders in perturbation theory. Furthermore, it produces the tree diagrams correctly. To get further insight into this new formulation it is necessary and important to understand the nature and the geometrical structure of the space $\mathrm{R}$. Following recent mathematical results on loop groups by G. Segal and G. Wilson, I and my collaborators were able to show that the space $\mathrm{R}$ can be modelled on an infinite dimensional manifold that is Grassmannian-like (Gr). In particular, we have shown that the partition function of QCSFT can be interpreted as the pull-back of a section of a holomorphic vector bundle whose base-space is $\mathrm{Gr}$. The new important and significant property of this bundle is that it is flat. The vanishing of its Ricci-two form can be interpreted as the field equation of QCSFT in which the dynamical variable is the hermitian metric.

The relevance of Grassmannians to the string program is potentially very great for the following reasons:

i) Grassmannians contain infinite genus Riemann surfaces which are believed to be relevant to non-perturbative string physics.

ii) Given that we can find measures in $\mathrm{Gr}$, we can then deduce the corresponding measure of QCSFT. This is of utmost importance for string perturbation theory. 
iii) It has been conjectured that string theory may be a completely solvable integrable system and the study of QCSFT within the context of Gr might lead to proof of this. This would be of the utmost importance because it would mean that the dynamics of QCSFT can be determined by solving certain non-linear differential equations (NLDE). In fact Grassmannians are the spaces of solutions of NLDE known as the KadomtsevPetviashvilli equations. The dynamical string picture obtained this way will be of non-perturbative nature. This will be vital for understanding the quantum geometry of space-time.

My plans for the future are to continue studying these intimate connections between Quantum String Field Theory, conformal field theory, algebraic geometry, and Grassmannians. In particular, I would like to achieve a genuine and proper consistent formulation of QCSFT.

\section{(h) S. Martin}

During the previous academic year I studied physics in two, three, and four spacetime dimensions. J. H. Horne (of UCSB) and I studied the form of the free energy in twodimensional gravity theories. We argued that the contributions to the free energy from higher genus surfaces can be written in terms of correlation functions on the sphere in a model independent way. This work appeared in Phys. Lett. B258, 322 (1991). I also studied topological field theories in thre dimensions. In a paper which will appear in Int. Jnl. Mod. Phys., I proposed a version of the canonical approach to such theories in which the fundamental fields of the theory are non-local variables associated with graphs, rather than gauge fields. More recently, I have been studying approaches to dynamical electroweak symmetry breaking in the Standard Model, especially the top-quark condensate idea originally suggested by Nambu. In a paper which will appear in Physical Reviev. D, I proposed a variation on the original top-quark condensate model, in which a third generation gauge singlet neutrino and the top quark both form condensates which break the electroweak symmetry. This model has the virtue tha the top-quark mass can come out in the range currently favored by indirect experimental constraints; in the original top-quark condensate model the prediction for the top-quark mass was evidently too large.

At present, I am finishing some work in which I discuss the possibility that the topquark condensate models can arise from a renormalizable anomaly-free Lagrangian. There are many constraints on such models, but a few of them may be capable of describing the real world. This work also indicates that in their renormalizable form, the top-quark condensate models are remarkably similar to extended technicolor models, and should be thought of in much the same way. I will probably continue to work on dynamical electroweak symmetry breaking for the foreseeable future.

\section{(i) C. Preitschopf}

In the academic year 1988/89 I studied superstring field theory, in collaboration with C. Thorn and S. Yost. The construction of a covariant and gauge-invariant superstring field theory that reproduced the known S-matrix had been an unsolved problem in spite of a number of proposals over the years. We were able to solve this problem on the basis of Witten's string field theory. We developed the requisite computational techniques for superghost systems, proved gauge invariance and explicitly com suted the tree-level $\mathrm{S}$-matrix. In developing the perturbation series we observed that the Chern-Simons form of the action has some interesting consequences: one can choose a gauge in which in some sense at tree level there are no interactions, so that most Feynman diagrams do not contribute to the manifestly nontrivial S-matrix. I believe that this aspect of string field theory is important and deserves further attention. The work on string field theory was written up in 1989 and published in Nuclear Physics B337 (1990) 363. 
In $1989 / 90$ the exciting developments in the matrix model appraoch to strings in one dimension or less led me to investigate Liouville theory. In less than 26 dimensions (or 10, in the supersymmetric case), one degree of freedom of the 2-d metric does not decouple and one has to quantize this Liouville field. This is a longstanding and difficult problem, which has not been solved in a satisfactory manner. It is an important step in linking up the critical string theory with the recent developments in the subcritical regime. I joined C. Thorn in an effort to use the subcritical dual model for open strings and the Bäcklund transform of the Liouville field to shed light on the theory in dimensions that are not yet accessible to matrix model methods. We were able to sharpen the distinction between the subcritical model in 25 dimensions and the critical string in 26. Our results are summarized in a string field action for subcritical open strings, and written up in the preprint UFIFT-90-17, which has been submitted to Nuclear Physics.

In parallel we developed further the theory of the Bäcklund transformation as it applies to the Liouville field in curved gravitational backgrounds. The transformation converts the interacting Liouville field into a free field, and I believe that the problem of computing correlation functions will most likely be solved via the free field representation. Maybe one can in this fashion even establish a link to topological gravity in two dimensions, and hence to the results of the matrix model approach. This work was written up in the preprint UFIFT-90-19, and was accepted for publication by Physics Letters.

In 1990-91 I followed up on some of the open problems in my work on subcritical strings and Liouville theory. There is the obvious problem of supersymmetrization. It becomes particularly interesting when the Parisi-Sourlas mechanism is operative and the effective dimension of our theory gets reduced. We might then be able to find an overlap between the region of validity of the matrix model technology and the string field theory approach.

If one considers supersymmetric strings, it becomes natural to take into account heterotic theories, i.e., left-right asymmetric ones, which in fact don't have to be supersymmetric. Then "subcritical" may refer in a different way to the left-moving sector and the right-moving one. If this is indeed the case, an additional component of the zweibein becomes dynamical and we have to deal with chiral quantum gravity.

I will try and extend the open subcritical string field theory to dimensions less than one. This will be part of a larger effort to rewrite the nonperturbative string equations derived with matrix model techniques as Schwinger-Dyson equations of string field theory. The string equations have been shown to be equivalent to loop equations, and of course string field theory is by definition a field theory of loops. The technical details of this equivalence, however, and the implications for more realistic string models have not been worked out.

Once it is clear what kind of string field theory we are dealing with, one should try and solve the vacuum problem: is it possible to compute the true ground state of the theory? At the moment the nonperturbative methods have not yielded new insight into this fundmental problem of string theory, but since we can solve some string models exactly, as well as shift their ground state, it should be possible to ask such a question and answer it at least in a suitably restricted sector of the theory.

I have now ended my stay at the University of Florida and now head for Gotesborg, Sweden for my future research studies.

\section{(j) S. Rey}

This was my last year as a Post Doc here in the High Energy Physics group and I focused my research on quantum field theory in general. I have become a post doc at 
Yale University. My past research covered various topics: weak interaction phenomenology, large scale structure of the universe, inflationary/quantum cosmology, string theory, quantum field theory, field theory application to condensed matter physics.

I continued investigating nonperturbative aspects of string theory: cosmological backgrounds, solitons and instantons, especially, based on conformal field theories. In field theory, solitons and instantons are characterized by various topological charges. Their quantization in terms of collective coordinates is well understood. I investigated such questions and various other related aspects of stringy solitons and instantons, in particular, in the context of exact conformal field theories.

I studied strings in external background fields. It provides an interesting duality between strong field/weak coupling and weak field/strong coupling that may be related to other string dualities (small/large radii and high/low temperatures) and to the nature of singularities in string theory.

I also investigate 4 various aspects of noncritical strings, especially, nonperturbat ve physics recently discovered by several groups. In particular, I focused on the breakdown of the perturbation expansion (condensation of handles), for example, by drawing an analogy to the ensemble of handles as a thermodynamics system. I also studied the nature of the transition across central charge $\mathrm{c}=1$.

Also, I investigated nontrivial gravitational solitons and instantons in $(2+1)$ dimensional Einstein gravity, analagous to their four-dimensional examples. I expect novel modifications of their properties once the gravitational Chern-Simons term is added.

\section{(k) S. Sin}

Last year I worked on the non-perturbative aspect of the 2-d gravity. In the work with Awada, I established that KP hierarchy posesses $W_{\infty}$ symmetry. In the same work I argued that $\mathrm{d}=1$ matrix model might be described by KP hierarchy, which is parallel to the fact that $d<1$ theories are described by the generalised KdV Hierarchies.

This year I am investigating following topics.

1. Completeness of the space of anyon wave function. The issue is that due to boundary condition imposed on the anyon wave functions many of the otherwise possible states are deleted hence there arises the question of the completeness of the space of the anyon wave functions.

2. Galaxy formation and early universe. With P. Sikivie I am studying

(A) Ripples of elliptic galaxy.

(B) Phase space theory of formation of galaxy and their classification.

Also with J. Hong and P. Oh I am studying early universe, specifically I ask whether the two step inflation can explain the hierarchy of scales in the obsevable universe.

3. Symmetry of Super KP hierarchy: whether SKP contains super $W_{1+\infty}$. (with E. Sezgin and A. Das).

4. The relation between 2 -d gravity and 4-d self dual gravity and the role of $W_{1+\infty}$ symmetry in both theories.

\section{(l) S. Yost}

This was my last year here at Florida and my interests while here were in string theories of fundamental interactions. I have taken a post doc position at the University of Tennessee. 
In this last year I worked on the exactly solvable case of strings in less than one dimension. I tried to understand the relation between two-dimensional topological gravity and the "matrix models" in more detail. There has already been much progress in solving various matrix models, but much can still be done to extract information of importance to string theory. In particular, it could be useful to develop a string field theory approach to topological gravity.

\section{(m) Y. Wang}

In the past two years, I did a lot of work in extended inflation, which is a new way of implementing inflation that is essential in solving the problems of the Standard Cosmology. My research in this area involved false vacuum decay and the building of successful extended inflationary models.

My present and future research concerns the interface between particle physics and cosmology. Currently I am investigating the formation of domain walls in a late-time phase transition that may seed the observed large-scale structure in the Universe. In the near future, I am interested in exploring certain particle physics models that may give rise to cosmological late-time phase transitions. I also plan to study the general constraints on cosmological models that arise from the large-scale structure in the Universe and the observed isotropy of the microwave background radiation. 


\section{ACTIVITIES OF THE PARTICLE THEORY GROUP (last several years)}

\section{A. Lectures and Seminars}

\section{Field}

Invited talk presented at the Workshop on Collider Phenomenology, Argonne National Lab, June 2-3, 1989.

Colloquium presented at Michigan State University, November 6, 1990.

Talk presented at the SDC Collaboration Meeting, Argonne National Laboratory, March 20-23, 1991.

\section{Ramond}

Participant, SSC workshop on the Standard Model, Dallas, Texas, May 1990.

Participant, XIX Conference on Differential Geometry in Physics, Rapallo, Italy, June 1990.

Seminar, University of Pennsylvania, September 1990.

Colloquium, University of Central Florida, Oct. 1990.

Invited Lecture, Sakita Symposium, CUNY, Oct. 1990.

Seminar, Johns Hopkins, May 1991.

SSC Colloquium, Dallas, Texas, May 1991.

Invited Participant XXth Conference in Differential Geometry in Physics, New York, June 1991.

Invited Lecturer, TASI Summer School, Boulder, Colorado, June 1991.

Invited Lecturer, Johns Hopkins Workshop, August 1991.

Seminar, University of Virginia, September 1991.

Invited Lecturer on the occasion of ECG Sudarshan's 60th birthday, Austin, Texas, September 1991.

\section{$\underline{\text { Sikivie }}$}

Astrophysics Seminar at Fermilab, February 1989.

Physics Department Colloquium at University of Alabama, Tuscaloosa, March 1989.

Particle Physics Seminar at LBL, March 1989.

Invited talk at the Cosmic Axion Workshop at BNL, April 1989.

Invited talk at the Symposium on Quantum Fluids and Solids, QFS 1989, Gainesville, April 1989.

Invited talk at 1st Workshop on Particle Astrophysics, San Miniato, Italy, May 1989.

Particle Theory Seminar at the University of Pisa, Italy, May 1989.

Invited talk at the Conference on CP Violation in Particle Physics and Astrophysics, Blois, France, May 1989. 
Invited talk at the 'DPF 90' meeting of the APS, in Houston, TX, January 1990.

Physics Department Colloquium at the Univ, of William and Mary, April 1990.

Seminar at Lawrence Livermore Laboratory, as part of 'Axion day at LLNL', May 1990.

Invited talk at the Nobel ' 90 Symposium on The Birth and Evolution of the Early Universe, Graftavallen, Sweden, June 1990.

Theoretical Particle/Astrophysics seminar at CERN, June 1990.

Short talk at the Aspen Workshop on Phase Transitions in the Early Universe, Aspen, CO, August 1990.

Invited talk at the annual meeting of SESAPS in Decatur, Georria, Nov. 1990.

Invited talk at the TEXAS/ESO-CERN Symposium on Astrophysics, in Brighton, England, Dec. 1990.

Lecture at the First International School on Particle Physics and Cosmology, in Baksan Valley (Caucasus), USSR, in May 1991.

Seminar at the Institute for Nuclear Research of the Academy of Sciences of the USSR, Moscow, May 1991.

Invited talk at 'Fezafest', a meeting in honor of Professor Feza Gürsey, at Yale University, May 1991.

Two lectures at TASI ' 91 , in Boulder, Colorado, June 1991.

\section{Thorn}

Theoretical Physics Seminar, Cornell, May, 1989.

Theoretical Physics Seminar, University of California, Berkeley, July 1989.

Theoretical Physics Seminar, Institute for Advanced Study, October, 1989.

Invited lecture, Univ. of Alabama, Tuscaloosa (String Workshop), November 1989.

Aspen Center for Physics, July-August, 1990.

Theoretical Physics Seminar, Imperial College, London, England, February 1991.

Theoretical Physics Semina*, Durham University, Durham, England, February 1991.

Theoretical Physics Seminar, Oxford University, Oxford, England, February 1991.

XVth Triangular Mieeting (Utrecht, Rome, Paris), April 3-5, 1991.

Theoretical Physics Seminar, Southhampton University, May 1991.

Theoretical Physics Seminar, Queen Mary College, London, England, May 1991.

DAMTP, Cambridge University, May 1991.

First International Sakhavov Conference on Physics, Lobedev Institute, Moscow, USSR, May 27-31, 1991.

Theoretical Physics Seminar, Karlsruhe, Germany, June 1991.

LMS Durham Research Symposium, Conformal Field Theory and Related Topics, July 9-19, 1991. 
Theoretical Physics Seminar, ENS, Paris, France, August 1991.

Qiu

Seminar, University of Florida, September 5, 1989.

Woodard

Seminar, Univ. of Florida, August 29, 1989.

Seminar, Univ. of Toronto, October 31, 1989.

Seminar, Syracuse University, Nov. 3, 1989.

Seminar, Univ. of Massachusetts at Amherst, Nov. 6, 1989.

Seminar, Brandeis University, Nov. 7, 1989.

Seminar, Brown University, Nov. 9, 1989.

Seminar, Univ. of California at Santa Barbara, May 18, 1990.

Seminar, Univ. of Florida, September 7, 1990.

Seminar, Brown University, October 18, 1990.

Seminar, Brandeis University, October 23, 1990.

Seminar, University of Florida, March 26, 1991.

Seminar, University of Crete, June 24, 1991.

B. Travel

\section{Field}

Dedication of the Feynman Computer Center, Fermilab, Dec. 1-2, 1989.

Fermilab PAC Meeting, Jan. 27-28, 1989.

Fermilab PAC Meeting, April 28-29, 1989.

Workshop on Collider Phenomenology, Argonne Nat. Lab., June 2-3, 19 ¿'$^{\prime}$.

Conference on Supercollider Physics and Experiments, Dallas, Oct. 1-4, 1!89.

DPF Executive Committee, Dallas, TX, Oct. 4, 1989.

DPF Executive Committee, Rice University, Jan. 3, 1990.

DPF Executive Committee, Washington APS, April 16-17, 1990.

DOE-LBL Review, Lawrence Berkeley Lab., May 30-31, 1990.

Snowmass Workshop, June 25-July 13, 1990.

SDC Collaboration Meeting, SSCL, Jan. 9-12, 1991.

SDC Collaboration Meeting, Argonne Nat. Lab., March 20-23, 1991.

SDC "Physics Subgroup" Meeting, SSCL, May 31-June 1, 1991.

SDC Collaboration Meeting, Berkeley, August 5-10, 1991. 


\section{Ramond}

Hepap Subcommittee, Hilton Head, SC, January 1989.

Caltech - M. Gell-Mann's Festschrift, January 1989.

Aspen Center for Physics, June-July, 1989.

Tuscaloosa, String Meeting, November 1989.

SSC Conference on Standard Model, Dallas, Texas, May 1990.

Conference on Differential Geometry, Rapallo, Italy, June 1990.

Ecole Normale Superieure, Paris, June 1990.

University of Pennsylvania, September 1990.

CUNY, October 1990.

University of Pennsylvania, May 1991.

Johns Hopkins, May 1991.

SSC Colloquium, May 1991.

HEPAP, Washington, Tune 1991.

XXth Conference in Dirierential Geometry in Physics, New York, June 1991.

TASI, Boulder, Colorado, June 1991.

Aspen Center for Physics, July 1991.

John Hopkins Workshop, August 1991.

University of Virginia, September 1991.

Austin, Texas, September 1991.

\section{$\underline{\text { Sikivie }}$}

Fermilab, Feb. 1989.

Univ. of Alabama, Tuscaloosa, Feb. 1989.

Lawrence Berkeley Laboratory, March 1989.

Brookhaven National Laboratory, April 1989.

San Miniato, Italy, May 1989.

Pisa, Italy, May 1989.

Blois, France, May 1989.

Aspen Center for Physics, Aug. 1989.

Houston, TX, Jan. 1990

Univ. of William and Mary, April 1990.

Lawrence Livermore Laboratory, May 1990.

Graftavallen, Sweden, June 1990.

CERN, Switzerland, June 1990. 
Aspen Center for Physics, August 1990.

SESAPS Annual Meeting, Decatur, Georgia, Nov. 1990.

TEXAS/ESO-CERN Symposium, Bright, England, Dec. 1990.

First International School Particles and Cosmology, Baksan Valley (Caucasus), USSR, May 1991.

Institute for Nuclear Rescarch, Moscow, May 1991.

Fezafest, Yale University, May 1991.

TASI '91, Boulder, Colorado, June 1991.

Aspen, Colorado, June 1991.

Thorn

Cornell University, May 1989.

SLAC, July 1989.

Inst. for Advanced Study, October 1989.

Univ. of Alabama, Tuscaloosa, November 1989.

Aspen Center for Physics, July-August 1990.

Imperial College, London, February 1991.

Durham University, Durham, England, February 1991.

Oxford University, Oxford, England, February 1991.

(Utrecht, Rome, Paris), France, April 1991.

Southhampton University, May 1991.

Queen Mary College, London, England, May 1991.

DAMTP, Cambridge Univ., Cambridge, England, May 1991.

Moscow, USSR, May 1991.

Karlsruhe, Germany, June 1991.

CERN, Geneva, Switzerland, July 1991.

Durham, England, July 1991.

ENS, Paris, France, August 1991.

Qiu

Rutger University, February 1990.

Texas A\&M University, March 1990.

\section{Woodard}

University of Toronto, 10/28/89-11/1/89.

Syracuse University, 11/2/89-11/3/89.

Univ. of Massachusetts at Amherst, Nov. 6, 1989. 
Brandeis University, Nov. 7, 1989.

Brown University, 11/8/89-11/11/89.

Univ. of California at Santa Barbara, 5/16/90-5/23/90.

Brown University, 10/18/90-10/21/90.

Massachusetts Institute of Technology, 10/22/90.

Brandeis University, 10/23/90

University of Crete, 6/11/91-7/7/91.

\section{Seminar Speakers and Visitors (recent)}

\section{Name}

Dr. Jim Hughes

Prof. Nathan Isgur

Prof. Rafael Nepomechie

Prof. L. McLerran

Prof. V. I. Ogievetskii

Prof. Alan Kostelecky

Dr. Michael Douglas

Prof. Jeff Harvey

Prof. Gabor Domokos

Prof. Luca Mezincescu

Prof. Rafael Nepomechie

Dr. Marti Ruiz-Altaba

Prof. B. Zwiebach

Prof. J. McCarthy

Prof. E. Braaten

Prof. A.Marshakov

Prof. A. Ashtekar

Prof. L. Perivolaropoulous

Prof. B. Holdom

Dr. J. Frieman

Dr. C. Crnkovic

Dr. L. Krauss

Prof. A. Jevicki

Prof. G. Horowitz

Prof. J. Distler

Prof. L. Romans

Prof. W. Siegel

Prof. J. Polchinski

Prof. D. Eliezer

Prof. E. Weinberg

Dr. C. Wendt

Prof. R. Brandenberger

Dr. J. Rosner

Prof. B. Ovrut

Prof. B. DeWitt

Prof. C. Vafa

\section{Institution}

Caltech

University of Toronto

University of Miami

University of Minnesota

Joint Institute for

Nuclear Research, Dubna

Indiana University

Rutgers University

Princeton University

Johns Hopkins University

University of Miami

University of Miami

CERIN

Mass. Inst. of Tech.

Boston University

Northwestern University

Princeton University

Syracuse University

Brown University

University of Toronto

Fermilab

Yale University

Yale University

Brown University

Univ. of California, S.B.

Princeton University

Univ. of Southern California

SUNY

University of Texas

Univ. of British Columbia

Columbia University

Fermilab

Brown University

Fermilab

University of Pennsylvania

University of Texas

Harvard University
Dates

$3 / 6 / 90$

$3 / 8 / 90$

$3 / 27 / 90$

$4 / 3 / 90$

$4 / 9 / 90$

$4 / 10 / 89$

$4 / 17 / 90$

$4 / 19 / 90$

$5 / 1 / 90$

$6 / 1 / 90$

$6 / 1 / 90$

$8 / 25 / 90$

$8 / 28 / 90$

$9 / 4 / 90$

$9 / 17 / 90$

$9 / 21 / 9$ ?

$9 / 28 / 90$

$10 / 6 / 90$

$10 / 15 / 90$

$10 / 23 / 90$

$11 / 12 / 90$

$11 / 15 / 90$

$11 / 17 / 90$

$11 / 22 / 90$

$12 / 3 / 90$

$12 / 8 / 90$

$1 / 5 / 91$

$1 / 9 / 91$

$1 / 12 / 91$

$2 / 11 / 91$

$3 / 9 / 91$

$3 / 16 / 91$

$3 / 28 / 91$

$3 / 30 / 91$

$4 / 14 / 91$

$4 / 29 / 91$

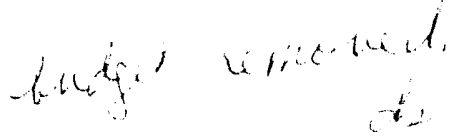




\section{Appendix A. GROUP PUBLICATIONS (last several years)}

1. Calculation of the one-loop graviton mass-shift in bosonic string theory, J. Minahan, UFTP-89-2, Nucl Phys. B333 (1990) 525.

2. Cavity design for a cosmic axion detector, P. Sikivie, D. Tanner, S.-I. Cho, UFTP-894, Rev. Sci. Instrum. 61 (1990), 1076-1085.

3. Algebra of Reparametrization-Invariant and Normal Ordered Operators in Open String Field Theory, P. Ramond, UFTP-89-5, Nuc. Phys. B333 (1990) 771-782.

4. Albelian Chern-Simons Theories in 2+1 Dimensions, A. Polychronakos, UFTP-89-7, Annals of Phys. 203 (1990) 231-254.

5. Alternative Ghost Structures for Superstring Field Theory, G. Kleppe and R. Viswanathan, UFTP-89-8, Nuc. Phys. B335 (1990) 589.

6. Abelian Chern-Simons Theories and Conformal Blocks, A. Polychronakos, UFTP-89-9, to appear in Annals of Phys.

7. Solar and Cosmic Axion Hunting, P. Sikivie, UFTP-89-10, published in the Proceedings of the 3rd Nishinomiya-Yukawa Symposium, Nishinomiya, Japan, Nov. 10-11, 1988. Also published in the Proceedings of the Workshop on Cosmic Axions, held at Brookhaven National Laboratory, April 13-14, 1989, edited by C. Jones and A. Melissinos, World Scientific, pp. 233-263.

8. Massive Condensates and Scaling in String Field Theory, D. Zoller, UFTP-89-11, Nuc. Phys B328 (1989) 545-556.

9. Algebra of Reparametrization-Invariant Operators in Open String Field Theory, $G$. Kleppe, P. Ramond and R. Viswanathan, UFTP-89-12, Nuc. Phys. B333 (1990) 771.

10. A New Non-Grassmannian Pseudo-Classical Action for Spin-1/2 Particles, R. Warner, UFTP-89-13.

11. Gravitational Particle Production by Cosmic Strings, J. Garriga, E. Verdaguer, D. Harari, UFTP-89-14, Nucl. Phys. B339 (1990) 560.

12. Stueckelberg, J. Minahan, UFTP-89-15.

13. A Comment on Anomaly Cancellation in the Standard Model, J. Minahan, P. Ramond and $R$. Warner, UFTP-89-16, Phys. Rev. D41 (1990).

14. The Strong CP Problem and the Axion P. Sikivie, UFTP-89-17, published in the Praceedings of the Conference on CP-Violation in Particle Physics and Astrophysics, held in Blois, France in May 1989, edited by J. Tran Thanh Van.

15. A Global String with an Event Horizon, A. Polychronakos and D. Harari, UFTP-89-18, Phys. Lett. B240 (1990) 55.

16. Superstring Field Theory, C. Preitschopf, S. Yost and C. Thorn, UFTP-89-19, Nucl. Phys. B337 (1990) 363.

17. The Orientation of the QCD Vacuum, P. Sikivie and C. Thorn, UFTP-89-20, Phys. Lett. 234B (1990) 132-134.

18. Open-Closed Transition for the NSR Spinning Strings, M. Chu, UFTP-89-21, Nuclear Physics B241 (1990) 37.

19. On the Quantization of the Coefficient of the Abelian Chern-Simons Term, A. Polychronakos, UFTP-89-22, published in Phys. Lett. B. 
20. A Classical Realization of Quantum Algebras, A. Polychronakos, UFTP-89-23, Modern Phys. Lett. A5 (1990) 2325-2334.

21. Scale Invariant Curvature and Massless Spinning Particles, D. Zoller, UFTP-89-24.

22. A Comment on Reparametrization Invariance in Witten's Open String Field Theory, G. Kleppe and R. Viswanathan, UFTP-89-25, Phys. Lett. B240 (1990) 80.

23. Subcritical Dual Models as Subcritical Strings, C. Thorn, UFTP-90-1, Phys. Lett. 242B (1990) 364 .

24. Non-Perturbative Higher Spin Gravity in Two Dimensions, Z. Qiu and M. Awada, UFTP-90-2, Phys. Lett. B in press.

25. Superstring Field Theory, C. Preitschopf, C. Thorn and S. Yost, UFTP-90-3, Nucl. Phys. B337 (1990) 363-433.

26. Non-Perturbative 2-d Quantum Gravity and the Generalized KdV Hierarchy, $M$. A wada and Z. Qiu, UFTP-90-4, Phys. Lett. B247 (1990) 71-74.

27. Fractional Charges on a String, J. Minahan, UFIFT-HEP-90-6.

28. Constraints on New Chiral Fermions in the Standard Model, J. Minahan, P. Ramond and B. Wright, UFTP-90-7, Phys. Rev. D42 (1990).

29. Nuclear Dipole Radiation from $\theta$ Oscillations, J. Hong, J. Kim and P. Sikivie, UFTP90-8, Phys. Rev. D42 (1990), 1847-1850.

30. Operator Quantization of the Green-Schwarz Heterotic String, M. Chu, UFTP-90-9, Nuclear Physics B360 (1991) 31.

31. The Critical Points of the Multimatrix Model as the Theories of 2-d W-Gravity, $M$. $A$ wada and Z. Qiu, UFTP-90-10, Phys. Lett. B245 (1990) 359-364.

32. Mass Reconstruction from Missing Transverse Energy and Jets, R. D. Field, UFTP90-11, submitted to Phys. Rev. D.

33. Study of Some Dual Solutions in 2+1 Dimensions, B. Keszthelyi, E. Piard and P. Ramond, UFTP-90-13, J. Math. Phys. $\underline{32}$ (1991), 210-222.

34. Consistency Conditions and Representations of a Q-Deformed Virasoro Algebra, $A$. Polychronakos, UFTP-90-14, Phy's. Lett. B256 (1991) 35-40.

35. Nonlocal Regularizations of Gauge Theories, D. Evens, J. W. Moffat, G. Kleppe and R. P. Woodard, UFIFT-HEP-90-15, Physical Review D43 (1991), 499-519.

36. Inconsistency of Scale Invariant Curvature Coupled to Gravity, D. Zoller, UFTP-90-16, Phys. Rev. Lett. 65 (1990) 2236-2238.

37. Action Principle for Subcritical String Fields, C. Preitschopf and C. Thorn, UFTP-9017, Nucl. Phys. B349 (1991) 132-158.

38. The Supersymmetric Modified KdV Hierarchy and Non-Perturbative Spinning String Theory in Less Than One Dimension, M. Awada, UFTP-90-18.

39. The Bäcklund Transform for the Liouville Field in a Curved Background, C. Preitschopf and C. Thorn, UFTP-90-19, Phys. Lett. B250 (1990) 79.

40. A Search for Cosmic Axions, C. Hagmann, P. Sikivie, N. Sullivan and D. Tanner, University of Florida preprint (March 1990), Phys. Rev. Lett. D42 (1990) 1297-1300.

41. A Supersymmetric Regulator, G. Kleppe and R. P. Woodard, UFIFT-HEP-90-21, Physics Letters B 253 (1991), 331-334. 
42. Nonlocal Yang-Mills, G. Kleppe and R. P. Woodard, UFIFT-HEP-90-22, submitted to Nuclear Physics B.

43. The q-Schroedinger, J. Minahan, UFIFT-HEP-90-23, Mod. Phys. Lett. A5 (1990) 2625-2632.

44. Cosmic Global Strings, P. Sikivie, UFIFT-HEP-90-24, published in Proc. of Nobel Symposium 90: The Birth and Early Evolution of Our Universe, Groftavallen, Sweden, June 11.16, 1990.

45. Nonlocal Regularization of Supersymmetric QED, G. Kleppe, UFIFT-HEP-90-25, Phys. Lett. B256 (1991) 431-436.

46. A Standard Model Extension with Neutrino Masses and an Invisible Axion, $P$. Ramond, B. Wright, and $B$. Arason, UFIFT-HEP-90-26, Phys. Rev. D43 (1991) 2337-2350.

47. Angular Momentum of an Electric Charge and Magnetically Charged Black Hole, $D$. Garfinkle and S. Rey, UFIFT-KEP-90-27.

48. Non-Critical Superstrings from Two-Dimensional N=1 Supergravity, Z. Qiu, UFIFTHEP-90-28.

49. The Supersymmetric Generalized Modified KdV Hierarchy and Odd Minimal Superconformal Field Theories Coupled to 2D Supergravity. II, $M$. A wada, UFIFT-HEP90-29.

50. Computer Simulations of the Motion and Decay of Global Strings, C. Hagmann and $P$. Sikivie, UFIFT-HEP-90-30.

51. Neutrino Masses in the Standard Model, $P$. Ramond, UFIFT-HEP-90-31, published in Symposium volume in honor of B. Sakita (World Scientific: Singapore 1991).

52. On the Form of the Free Energy in 2-D Gravity Theories, J. Horne and S. Martin, UFIFT-HEP-90-32, Phys. Lett. B258 (1991) 322-330.

53. Twisted W-infinity Symmetry of the KP Hierarchy and the String Equation of $\mathrm{d}=1$ Matrix Models, M. Awada and S. Sin, UFIFT-HEP-90-33, submitted to Phys. Lett. B.

54. Topological $\mathrm{N}=1$ Superstrings in $\mathrm{D}<$ infinity, Z. Qiu and S. Rey, UFIFT-HEP-90-34.

55. Quenched $\mathrm{c}=1$ Critical Matrix Model, Z. Qiu and S. Rey, UFIFT-HEP-90-35, Phys. Lett. B264 (1991) 79-84.

56. The Graph Representation Approach to Topological Field Theory in $2+1$ Dimensions, S. Martin, UFIFT-HEP-91-2.

57. The String Difference Equation of $d=1$ Matrix Model and $W_{1+\infty}$ Symmetry of the KP Hierarchy, M. Awada and S. J. Sin, UFIFT-HEP-91-3, submitted to Phys. Lett. B.

58. Two Loop Calculations Using Nonlocal Regularization, G. Kleppe and $R$. Woodard, UFIFT-HEP-91-4.

59. Stringy Domain Walls and Target Space Modular Invariance, M. Cvetic, F. Quevedo and $S$. J. Rey, UFIFT-HEP-91-5, submitted to Phys. Rev. Lett.

60. Superconformal Field Theory of Axionic Instantons and Axionic Strings, S. J. Rey, UFIFT-HEP-91-6.

61. A Classical Theory of Continuous Spin and Hidden Gauge Invariance, D. Zoller, UFIFT-HEP-91-7. 
62. Dynamical Electroweak Symmetry Breaking with Top Quark and Neutrino Condensates, S. P. Martin, UFIFT-HEP-91-8.

63. Classical Fluids of Negative Heat Capacity, P. T. Landsberg and $R$. $P$. Woodard, UFIFT-HEP-91-9.

64. Semirigid Construction of Topological Supergravities, S. Rey, S. Govindarajan, and $P$. Nelson, UFIFT-HEP-91-10.

65. Construction of Lax Operators from Weight Diagrams, S. Carbon and E. Piard, UFIFTHEP-91-11.

66. Supermatrix Models, S. Yost, UFIFT-HEP-91-12.

67. Metaplectons' and Fractional Statistics, D. Castaño, UFIFT-HEP-91-13.

68. A Higher Derivative View of the Arc Length and Area Actions, D. Zoller, UFIFTHEP-91-14.

69. Strong Infrared Effects in Quantum Gravity, N. C. Tsamis and R. Woodard, UFIFTHEP-91-15.

70. Top Quark and Higgs Mass Bounds from a Numerical Study of Super-GUTs, H. Arason, D. Castaño, B. Keszthelyi, S. Mikaelian, E. Piard, P. Ramond and B. Wright, UFIFT-HEP-91-16, to appear in Phys. Rev. Lett.

71. Phase-Space Structure of Cold Dark Matter Halos, $P$. Sikivie and J. Ipser, UFIFTHEP-91-17.

72. Two Loop Calculations Using Nonlocal Regularization, G. Kleppe and R. Woodard, UFIFT-HEP-91-20.

73. Physical Green's Functions in Quanturn Gravity, N. Tsamis and R. Woodard, UFIFTHEP-91-21.

74. SDC Solenoidal Detector Notes, Jets in the Forward Region, R. Field, M. Barnett, and $A$. White, UFIFT-HEP-91-22.

75. Reformulating String Theory with the 1/N Expansion, C. B. Thorn, UFIFT-HEP-9123.

76. Renormalizable Top-Quark Condensate Models, S. Martin, UFIFT-HEP-91-24.

B. Conference Reports

77. Global Cosmic Strings, $P$. Sikivie, to be published in the Proceedings of the Nobel '90 Symposium.

78. Superstring Field Theory, C. B. Thorn, invited lecture at the Workshop on Superstring and Particle Theory, Tuscaloosa, Alabama, Nov. 8-11, 1989, L. Clavelli and B. Harms, Eds., World Scientific (1990), pp. 38-48.

79. Two lectures on Neutrinos, P. Ramond, two lectures delivered at TASI '91, Boulder, $\mathrm{CO}$, to appear in proceedings, UFIFT-HEP-91-25.

80. Cosmic Axion Searches, at the Annual Meeting of the South Eastern Section of the American Physical Society, Decatur, Georgia, July 17, 1990; and at the Texas/ESOCERN Symposium, Brighton, England, Dec. 12-16, 1990.

81. Reformulating String Theory with the $1 / N_{c}$ Expansion, invited talk at the First International A. D. Sakharov Conference on Physics, P. N. Lebedev Institute, Moscow, May 27-31, 1991. 


\section{Research in Experimental High Energy Physics}

Task B

Principal Investigators:

Paul Avery and J. Yelton

Physics Department

University of Florida

Gainesville, Florida 32611 


\section{Introduction}

UF Task B has been funded continuously by DoE since 1986. For the year 1991-92 it was decided to split the previous task into two, with J.K.Walker (hardware development for SSC experiments) splitting off into a separate task. In summer 1991 A. White left UF to be a Full Professor at University of Texas (Arlington) where he is establishing a new High Energy Experimental group and will continue to work with D0 and SDC collaborations. We are hoping to replace him with a new Research Scientist, and the Department has committed a half line to High Energy Physics for this purpose. In the meantime, as soon as the ICD project is complete, the UF participation in D0 will be over. R. Stephens, our post-doctoral research associate on D0 has transferred his energies to the CLEO experiment.

R.D. Field has collaborated with A.White on the software of SDC and has proposed a new task to continue this work. P.Avery has recently joined SDC and plans to carry out software studies as a natural extension of the work that he has already done as a SAHEP committee member. In addition, he will be collaborating with Rick Field on physics simulations, with and without detectors. In this way we hope that our connection with SDC will remain healthy and form a basis from which a larger group may grow.

The Chairman of the Department of Physics has set up a committee to evaluate the best way to ensure strong growth in the UF High Energy physics program in the next few years. Unfortunately the State of Florida budget crisis has made it impossible to predict when new faculty hires can be made. However the Dean of the College of Liberal Arts and Sciences, the Department Chairman and the Faculty are all committed to the growth of the HEE program as soon as State funding of faculty lines can be obtained.

Although the State is in a severe financial crisis, the Physics Department has managed to win support from the State for a new building. Present plans are for a completely new building large enough to house the entire Department, and including a large laboratory where it will be possible to build large scale HEE detectors. The funding for the building is greater thatn $\$ 30 \mathrm{M}$ and it should be complete in 1995-96.

\section{The D-zero Experiment at Fermilab}

During the past year the University of Florida has made many contributions to the hardware, software and analysis efforts the D-Zero experiment. Our primary focus has been the construction, installation and software implementation of the Intercryostat detector. Major contributions have also been made in the area of software development for physics analyses. Details of these, and other, contributions are given below. 


\subsection{The Intercryostat Detector (ICD)}

\section{1a Hardware (R.Stephens and A.White}

This detector was developed for the purpose of correcting for energy losses in the inactive material between the central and end calorimeter systems. The concept of the detector resulted from Monte Carlo studies at the University of Florida (UF) and has been built and will be installed jointly with the University of Michigan (UM).

The design of the ICD was completed last year. It is composed of scintillator tiles (BC414) with bundles of wave-shifting fibers glued into grooves cut into the tiles. Each tile covers a tower of (azimuthal angle, pseudorapidity) per the D-Zero design of $(0.1,0.1)$. The tiles are held in metal boxes which also hold the photo-multiplier tubes, pre-amplifiers, and laser-calibration fibers.

The construction tasks were set up so that UF would build half of the tiles, the boxes, associated hardware, octant assembly jig and mounting/installation apparatus; while UM would build the other half of the tiles and supply the electronics and calibration system.

We, at UF, assembled a production crew of six undergraduate students. With our crew, and in coordination with UM, the production process was debugged and perfected. Both UF and UM will have produced 425 tiles each by September's end -- as this is written UF has produced 367 tiles. The tiles consistently yield 1.5 photo-electrons per minimum ionizing particle -- some tiles deliver as many as 28 and tiles that yield less than 7 are rejected.

ICD tiles have been exposed (UM) to the D-Zero test beam at Fermilab as part of the comprehensive calorimeter testing program. An array of tiles was set up between central and end calorimeter modules in the cryostat containing liquid argon. Metal plates were included to simulate the dead material of the end and central cryostat walls in the actual D-Zero detector. The test beam results have shown that the correlations between the ICD signals and the energy deficit in the live calorimetry, predicted by our Monte Carlo studies, do indeed exist. The observed correlations are in fact somewhat better than predicted by Monte Carlo.

The design of the boxes was completed after prototypes of the electronics and tules v, ere studied. The box construction was contracted out to Omega metals -- a sheet metal fabrication company located in Keystone Heights, Florida. The boxes were delivered in December of 1990.

Since the ICD will be installed as eight octants forming a ring on the end-cap calorimeter (EC), and due to the difficult geometry (half of the ring concave at one radius and the other convex at a different radius) it was important to have an octant assembly jig which afforded us the ability to quickly and easily assemble octants. The design of this jig was submitted to the 
shop of UF. The jig was delivered in June, the jig allows us to assemble octants in less than 15 minutes.

Because of the flexible nature of the octant design, but the inflexible character of the end cap calorimeter, it was imperative that we have a mounting/installation apparatus which would hold the octants rigidly in the proper shape as the octants were carried from the assembly jig to the EC. The apparatus was designed to hold the octants to within about 50 mils of the positions of mounting studs on the EC. Drawings of the mounting/ installation apparatus were submitted to UF's shop and two such upparati were produced. They consist of square aluminum-alloy piping welded into a trapezoid with quarter-inch thick tabs welded to it so that the octant attaches to the tabs and is held rigidly in the desired shape to the precise tolerance at six different points.

The octant assembly jig and the mounting/installation apparati have been used to successfully and conveniently mount six octants to D-Zero's north EC.

The completion of mounting and installation will be accomplished thiough the collaboration of A.P. White at the University of Texas at Arlington and our colleagues at UM.

\subsection{Software (R. Stephens)}

A versatile software package was developed to enable the experimentor to easily configure a variety of schemes to correct the observed energy for losses in the inactive material. Three distinct techniques of varying complexity are employed which utilize correlations of observed energies in various layers of the active calorimetry and the ICD. The package both derives, as a stand alone program, and implements, as an integral part of offline calorimeter reconstruction, the corrections based on the experimentor's desire.

The studies involved in the development of this software resulted in the D-Zero notes 1164 , "User's Guide to CC/EC Energy Correction Software", and 1165, "Energy Corrections For the CC/EC Transition Region".

\subsection{Physics Analysis}

\section{3a D-Zero Geant Monte Carlo on the VAX Farm (R.Stephens)}

The UFMulti system was employed in production runs of D-Zero Monte Carlo events. Over 8,000 events from the D-Zero "Double Blind Challenge" sample of $10 * * 5$ fake data -- an exercise to challenge the collaboration to tune up analyses techniques in anticipation of real data -- were run through the system using about a CPU hour per event. 
In coordination with the double-blind challenge, a sample of $10^{* * 5}$ background $\mathrm{QCD}$ events have been produced and 4,000 of them were put through the UFMulti system running DZero Geant.

\section{3b Particle Algorithm Group on Missing Transverse Energy (A. White)}

A. P. White heads the D-Zero subgroup which develops algorithms for the reconstruction of missing transverse energy (MET). As the leader of the sub-group he coordinates the various simultaneous efforts: 1) development of MET corrections (ICD, muon, ...);2) understanding the D-Zero MET resolution; 3) comparisons of D-Zero MET resolutions to other experiments; and, 4) use of MET in physics analyses.

The software package developed by R.W. Stephens for the energy corrections discussed above was initiated as a study of ways to increase the MET resolution.

\section{3c QCD/New-Physics Working Group (A. White, R.Stephens)}

A.P. White co-chairs the QCD/New-Physics working group of D-Zero in coordination with H. Weerts of Michigan State University, White's tasks in this capacity include: 1) coordinating physics analyses with the aim of avoiding duplicity and pursuing variety in the schemes employed to seek out signals of previously unobserved physical processes; 2) dividing thesis topics among the graduate students of various institutions whose interests lie in the area of heretofore unobserved physics with equity; 3 ) keeping the collaboration at-large, as well as the leaders of the other physics sub-groups, informed of the needs and desires of the QCD/NewPhysics sub-group; and, 4) coordinating trigger studies for "New-Physics" to prepare the trigger scheme for initial D_Zero data taking.

Participation in the "Double-Blind Challenge" has lead the "New-Physics" group to develop analyses meant to seek out "New" physics using well understood Supersymmetry signatures as a guide. From the standard SUSY signals of missing transverse energy in multi-jet events and the gold-plated signal of like-sign dimuons plus multi-jet events to signals of onsets of wholly unexpected new physics, original techniques are being developed. Members of the group have presented results using these techniques on the Double-Blind events at the collaboration's two Monte Carlo mini-conferences that have been held in the last year.

Results on supersymmetric particle production at D-Zero were also contributed to a study of b' production lead by Daria Zieminska of the University of Indiana. It was shown that, while SUSY and b' signatures are similar, there are techniques available to separate them while preserving adequate statistics for each process for further study. 


\subsection{Conclusion}

The University of Florida's participation in the D-Zero experiment will terminate when the last of the ICD tiles has been manufactured and delivered to the University of Michigan. A.P. White will continue with the effort from the University of Texas at Arlington and R.W. Stephens has joined the University of Florida's efforts with the CLEO collaboration.

\section{The CLEO Experiment}

\subsection{Introduction and History}

The CLEO experiment resides at the south end of the Cornell Electron Storage Ring (CESR). The detector is used to study $\mathrm{e}^{+} \mathrm{e}^{-}$interactions at center of mass energies near $10 \mathrm{GeV}$. Much of our effort has focussed on the properties of the $Y$ family of resonances (a total of 6 have been discovered), particularly the $\Upsilon(4 S)$ since it decays into mesons carrying a single b quark.

The energy range is also well suited to studies of charmed mesons and baryons. This has become an increasingly fruitful area of the research for CLEO now that the SPEAR facility as SLAC has been closed dowr. The CLEO collaboration now comprises around 140 professional physicists and graduate students from 17 institutions ( Caltech, Carnegie-Mellon, Colorado, Cornell, Florida, Harvard, Kansas, Minnesota, Ohio State, Oklahoma, Purdue, Rochester, San Diego, Santa Barbara, SUNY-Albany, Syracuse, and Vanderbilt). Carnegie-Mellon has rejoined, and U.C. San Diego is a new member this year. Florida has been a member since September, 1985

CLEO completed a $\$ 36$ million upgrade program funded by NSF in 1989. The 1987-88 data set was taken with a new tracking system (main drift chamber and vertex chamber), this configuration was known as "CLEO 1.5". Since then there have been further improvements in the inner detector together with a large "crystal calorimeter" which dramatically improves the detection and measurement of neutral particles. The detector, in its new and improved form, is known as "CLEO II". The detector has been running in CLEO II for almost two years. In the 1991 summer conferences many new results were presented that took advantage of the crystal calorimeter. In the meantime, upgrades to CESR have led to large increases in luminosity. The data sample analyzed for the summer conferences is approximately twice the size of the CLEO 1.5 data sample. Unfortunately the summer saw a long shutdown for a dedicated synchrotron radiation run, but in September the machine turned on again for CLEO physics. The long 
shutdown allowed a major realignment of the magnets in the ring, and new cavities installed (the "MARK III" cavities) give hopes of further large increases in luminosity.

\subsection{Summary of Work at CLEO: Oct. 1990 - Sep. 1991}

\section{2a Physics Analysis at CLEO}

When CLEO 1.5 stopped HEP running in April 1988. A total of $212 \mathrm{pb}^{-1}$ of data had been taken at the $\Upsilon(4 \mathrm{~s})$ resonance, $102 \mathrm{pb}^{-1}$ just below the $\Upsilon(4 \mathrm{~s})$ resonance, and $114 \mathrm{pb}^{-1}$ at the $\Upsilon(5 \mathrm{~s})$, The first CLEO II running was at the $\Upsilon(3 \mathrm{~s})$; not the first choice of the CLEO collaboration, but as it is the final days of the CUSB dectector they had the first choice of the energy. After the $\Upsilon(3 \mathrm{~s})$ run CLEO chose to run first above the $\Upsilon(4 s)$ and then at the $\Upsilon(4 s)$. The former energy was chosen for studies of $\mathrm{BB}^{*}$ production, the latter is optimal for $\mathrm{B}$ reconstruction. In all over $800 \mathrm{pb}^{-1}$ of data have been taken; already a large increase on the old data sample.

Although the collaboration has expanded greatly over the last few years, there has still been a lack of manpower in some areas. This is because the collaboration has three different tasks, Firstly the last papers from the CLEO 1.5 data have to be published. Secondly, there is a huge amount of new data to be processed and fully analyzed. Thirdly, there is a large effort to get a complete rebuilding of the CESR machine (i.e. a "B-Factory"). Many of the new collaborators work primarily on this project and the associated detector considerations. In the meantime the running of the detector requires two people on shift, 24 hours a day, and of course a lot of hard work to keep all aspects of harware fully operational.

In the last twelve months the following papers have appeared in journals.

1. Measurement of the $\Lambda_{t}$ Decay Parameter Asymmetry. J.Alexander et al., PRL 65, 2842 (1990).

2. Study of $\pi \pi$ transitions from the $\Upsilon$ (3s). I. Brock et a?., Phys. Rev. D43, 1448 (1991).

3. Study of $D^{\circ}$ decays into $\pi^{\circ}$ 's and $\eta^{0}$ 's. K.Kinoshita et al., Phys. Rev. D43, 2836 (1991). This analysis was performed by Florida.

4. $\Lambda_{c}^{+}$Production in $e^{+} e^{-}$Annihilation. P.Avery et al., Phys. Rev. D43, 3591 (1991). part of this analysis was performed by Florida.

5. Study of $\mathrm{D}^{*+}$ Spin Alignment. Y.Kubota et al., Phys. Rev. D44, 593 (1991). 
6. Exclusive and Inclusive Decays of B $\rightarrow$ D. R.Fulton et al., Phys. Rev. D43, 651 (1991).

7. Study of $\mathrm{K}^{*}$ Production in $\tau$ Decays. M. Goldberg et al., PRL. CLEO have the largest $\tau$ sample ever collected, and this analysis measures, with much greater precision than was ever previously possible, these Cabbibo suppressed decay modes. This analysis was performed by Florida.

8. Exclusive and Inclusive Semi-leptonic Decays of B $\rightarrow$ D. R.Fulton et al., Phys. Rev. D43, 651 (1990).

9. Study of $D \rightarrow K R$ and $\pi \pi^{-}$. J.Alexander et al., PRL 65, 1184 (1990). This is the best measurement of the ratio $\mathrm{K}^{+} \mathrm{K}^{-} / \pi^{+} \pi^{-}$; it remains a mystery why it is not closer to 1 . This analysis was performed mostly by Florida.

10. Determination of $\mathrm{B}\left(\mathrm{D}_{\mathrm{S}} \rightarrow \phi \pi\right)$. J.Alexander et al., PRL 65, 1531 (1990).

The following papers are available as CLNS notes (i.e. preprints).

1. Measurement of Unusual Decays of D Mesons Decays into Charged Particles. This is a series of new measurements of unusual modes, some never previously seen. This analysis was performed by Florida. R.Ammar et al., CLNS-91-1058.

2. Inclusive and Exclusive Decays of B Mesons to Final States including Charm and Charmonium Mesons. D. Bortoletto et al., CLNS-91-1102.

3. Semi-Leptonic B-Meson Decays at the $\Upsilon(4 s)$. S. Henderson et al,, CLNS-91-1101.

4. Inclusive $\chi_{b}(2 p)$ Production. R. Morrison et al., CLNS-91-1091.

5. Inclusive $B^{*}$ Cross-Section above the $\Upsilon(4 s)$. M. Alam et al., CLNS-91-1089.

6. Measurement of the ratio $B \rightarrow K^{*} e^{+} v / B \rightarrow K^{-} e^{+} v$. G. Crawford et al., CLNS-91-1083.

In addition the following papers were submitted to the lepton-photon conference in Geneva, July 1991. Most of these papers will form the basis of PRL publications in the forthcoming months. 
1. Electronic Branching Ratio of the $\tau$ Lepton.

2. A Precision Measurement of the $\tau$ Lifetime.

3. $\tau$ Decays to Dingle Charged Hadrons plus $\pi^{0}$ 's.

4. Decays of the $\Lambda_{c}$ to Final States with Photons. Florida was involved in this analysis.

5. Study of $D^{0}$ Decays to Neutral Particles. Part of this analysis was performed by Florida.

6. $\Lambda_{c} \rightarrow \mathrm{X \Lambda lv}$, and a Normalization of $\Lambda_{\mathrm{c}} \rightarrow \mathrm{pK} \pi$

7. Measurement of the $D^{*}(2010)$ Branching Fraction.

8. Two-Body $D_{s}$ Decays to $\eta \pi^{+}, \eta^{\prime} \pi^{+}, \eta \rho^{+}, \eta^{\prime} \rho^{+}$and $\eta \rho^{+}$.

9. Observation of the Decay $\Xi_{c}^{0} \rightarrow \Omega^{-} \mathrm{K}^{+}$.

This analysis was performed by Florida.

10. $\psi$ Production in $\mathrm{e}^{+} \mathrm{e}^{-}$Annihilations in the $\Upsilon(4 \mathrm{~s})$ Energy Range.

11. Inclusive and Exclusive Decauys of B Mesons to $\chi$.

12. Search for Electromagnetic Penguin Mediated B Decays.

13. Analysis of Hadronic transition in Decays of the $Y(3 \mathrm{~s})$.

14. Fragmentation Studies in the $10 \mathrm{GeV}$ Energy Range.

3.3 Florida's Activities at CLEO: Oct. 1990 - 1991

\section{3a Physics analyses}

Florida has made a major contribution to the physics analysis as descibed in the first part of section 4. This describes the work that has has reached the stage of external talks and publications. Of course there is much work in progress. This includes $\Omega^{-}$production in $\Upsilon(1 \mathrm{~s})$ decays and in continuum; we have the largest sample of $\Omega^{\prime}$ s in $\mathrm{e}^{+} \mathrm{e}^{-}$annihilation data, and noone 
else has accurately measured the number of $\Omega$ 's in continuum. This analysis has been presented in a CBX note. Several of the group are working on B-reconstruction - attempting to increase the yield of fully reconstructed $B$ decays. Work in the decay mode $D^{*} \pi(\pi)$ was presented by CLEO representatives at the summer conferences. At present Ame Freyberger is working on using CLEO II to measure Cabibbo-suppressed semi-leptonic charm decays, in order to measure $\mathrm{V}_{\mathrm{cd}} / \mathrm{V}_{\mathrm{cs}}$. John Yelton is working on new unusual decays of $\Lambda_{\mathrm{c}}^{+}$, in particular the new decay mode $\Lambda \mathrm{K}_{\mathrm{S}}^{0} \mathrm{~K}^{+}$found in both old and new data, that will form a part of a new $\Lambda_{\mathrm{c}}^{+}$decay mode paper. Jorge Rodriguez and Paul Avery are looking at large numbers of fully reconstructed $B$ events with a view to looking at the properties of the recoil particles to a B meson. They are also working on rare decays of the B into two charmed particles.

\section{3b CLEO II Pass 2 data reduction}

We have converted all our code to run on our large UNIX computing system, including using the UFMULTI multi-processing package to analyze the large CLEO II data sample. We used our UNIX power to run the PASS 2 processing (i.e. event reconstruction). First we had visitors at Cornell to help set up the processing chain here. Then Jorge Rodriguez took responsibility to complete the task, with help from student programmers and of course our system's manager. In all $400 \mathrm{pb}^{-1}$ of data were analyzed in this fashion (about half of the complete CLEO II data sample). Furthermore, we loaned some of our DECstations to Cornell to perform another large fraction of PASS 2, so in all around $75 \%$ of the processing was done on Florda's machines.

\section{3c Standard Monte Carlo generation}

Jorge Rodriguez is also in charge of local generation of Monte-Carlo programs using the GEANT description of the CLEO II apparatus. CLEO now has a Monte-Carlo coordinator based at Cornell, he finds out what Monte-Carlo generation is wanted, and asks a local coordinator to gentrate the events. They then get taken by tape back to Comell to be available for all users. Jorge Rodriguez has developed the standard files to automate the generation, and has so far generated around 30,000 events using the Florida machine. We have contracted to generate the largest fraction of Monte Carlo events on our Unix farm.

\section{3d CLEO Librarian}

Ame Freyberger has become the CLEO librarian. He is responsible for collecting together all additions and corrections to the CLEO libraries, and installing them in the CLEO system. This code then has to be tested, and if necessary debugged. The task is made more difficult by 
the fact that much of the code has to have different versions for VAXstations, DECstations and SGI machines.

\section{3e CLEO B Factory work}

All members of the Florida group have taken important roles in the proposal for a BFactory at Cornell. In January 1991 there were three documents put together. They were "The Physics Rationale for a B-Factory", the "Detector for a B-Factory" and the "Conceptual Design for a B-Factory based at CESR". For the first, John Yelton wrote the chapter on charm studies this included some Monte-Carlo work on separating D- $\bar{D}$ mixing from doubly-Cabibbo suppressed decays. Arne Freyberger worked on several aspects of the proposal, in particular simulations of the $\mathrm{B} \rightarrow \psi \mathrm{K}^{*}$. He also provided the neutral detection package for the simulation. Paul Avery oversaw the entire simulation effort at Cornell and presented results at conferences.

\section{3f CLEO software}

UF has written a large fraction of the physics software that has become the standard for CLEO analysis in the past year. First, we provided the standard $\mathrm{dE} / \mathrm{dx}$ correction that is applied to all charged particles in CLEO, thu i ensuring that all our invariant mass plots peak at the right place. Second, we defined standard procedures for combining charged tracks, showers, piOs, VOs and virtual particles into a common framework (the $\mathrm{KN}$ list). This step is important for CLEO II because of the many kinds of decay modes that must be considered. We are pushing the unification in preparation for a more ambitious kinematic fitting program described in the next section.

Third, we wrote the software superstructure DRIVER which all CLEO members use for their physics analyses. Fourth, we wrote a general package (PHYROAR) which has the ability to perform general looping, thus greatly simplified physics analysis when many different decay modes are analyzed at once. PHYROAR is meant to be a stopgap until a more comprehensive package - described below - is developed. Finally, we provided a comprehensive set of veriex constraint packages, including routines which allow the vertex to be constrained to a particular plane, line or point.

\subsection{Long term projects}

We are involved in several long term projects, both singly and in collaboration with others, that will significantly change the way high energy physics data is analyzed in the future.

\section{4a The CABS project}


We are working with Nobu Katayama and Dan Riley of Cornell in extending the physics analysis package CABS (CLEO Analysis by Script) written by Katayama (see talk at 1991 Computing Conference in Japan). This system is becoming very popular at Comell as an analysis tool. Our intention is to fully integrate kinematic fitting (written by Avery) and multiprocesing to make CABS a complete analysis system similar in spirit to PAW but much more advanced. As it is currently written, a user provides a file written in "Script" describing the analysis to be carried out directly in terms of physics concepts. A program (compiler) translates this information into $\mathrm{C}++$ which is then compiled on the host machine and executed.

Being a preprocessing system, CABS suffers from the lack of debugging tools that plague all such systems. We are proposing to provide direct programming support for the physics concepts but in such a way that the user can use $\mathrm{C}++$ directly rather than through the Script file. This provides a learning path: Use the Script language until you are familiar with $\mathrm{C}++$ and then use $\mathrm{C}++$ directly. We expect that most users will elect to stay with the Script approach.

We intend to make CABS available for general HEP use, although probably not during 1992 because of the magnitude of the task.

\section{4b The GISMO project}

We began collaborating in May, 1991 with physicists from SLAC and CERN on a project to develop a new Monte Carlo Simulation package. The project is called GISMO and is led by Bill Atwood of SLAC. The intention is to develop a simulation package based on object oriented principles. About 20 or so physicists from SLAC, CERN and several scattered institutions are involved. Working code was originally developed on NeXT machines written in Objective-C, an extension to $C$ that permits object oriented programming (OOP) constructs. The long term goal of GISMO is to eventually replace GEANT, although this will certainly not happen in time to affect the next round of SSC proposals. GISMO's goals and our role in achieving them are described in detail in our Task S proposal.

\section{4c The kinematic fitting project}

High energy physics has not had a general kinematic fitting package since SQUAW was written in the 1960's. SQUAW suffers from several problems: (1) its interface is not well suited to modern HEP experiments and (2) it does not provide sufficient support for vertex constraints, 
since it does not allow unknown vertices to be evaluated during a fit. Other kinematic fitting packages are usually specific to the experiments for which they were written and also do provode correct support for unknown vertices.

To address these problems, Avery has begun writing a new kinematic fitting package that will be general enough to be interfaced to other experiments and contain suport for calculation of vertices in conjunction with other constraints. This new system will return more information than the standard chisquare, enabling a user to make cuts on portions of the chisquare which are known to be background prone. The package is being written in $\mathrm{C}++$, which permits the use of more modem computing techniques such as inheritance, data and method encapsulation and extensibility.

\section{4d The UFMulti project}

The UFMulti software system we developed at UF has been the cornerstone of our computing effort since 1988. With UFMulti a single user can use the entire computing capability on a network, even when it is in the form of individual workstations. This has allowed us to take advantage of the microprocessor revolution to acquire a large amount of computing power at low cost, because we do not need the expensive high bandwidth buses used in almost all parallel processor designs. Our development of UFMulti also helped generate funding for computer resources and enabled us to negotiate better discounts from computer vendors.

During the past year we ported UFMulti to Unix and can now routinely run HEP jobs using 15-25 processors for rapid turnaround time. However, we believe UFMulti is too limited and, after much though, we have decided to completely rewrite it. This decision is based on what we have leamed in the past three years about computing, particularly distributed processing and object oriented programming. Accordingly we will write UFMulti in $\mathrm{C}++$ (it is currently written in Fortran and C) but it will remain callable from $\mathrm{C}$ or Fortran. Using $\mathrm{C}++$ will make JFMulti extensible (i.e., users can easily add features to it) and enable us to better integrate it with GISMO and CABS, both of which are written in C+t. How we intend to carry out this rewrite is described in more detail in the Task $S$ writeup. We will perform this work in collaboration with the SSC Computing Division. 


\title{
PILOT EXPERIMENT TOWARD A LARGE-SCALE DARK-MATTER AXION SEARCH
}

\author{
P. Sikivie, N. S. Sullivan, and D. B. Tanner \\ Department of Physics, University of Florida, Gainesville, FL 32611
}

\begin{abstract}
The axion occurs in two of the most important questions in all of science, namely, the origin of $\mathrm{CP}$ symmetry in the strong interactions and the composition of the dark matter of the universe. First predicted in 1978, present laboratory, astrophysical, and cosmological constraints place the axion mass in the $1 \mu \mathrm{eV}-1 \mathrm{meV}$ range. Axions are especially significant as dark matter if their mass is around $1 \mu \mathrm{eV}$. These axions may be detected by their coupling to photons through the $\mathbf{E} \cdot \mathbf{B}$ interaction in a tunable high- $Q$ microwave cavity permeated by a strong external magnetic field. Two pilot experiments have already reported results from this mass region, but fall short of making the definitive measurement by 3 to 4 orders of magnitude in power sensitivity. We propose continued research into the development of cavity detectors, focussing on the problems of tuning and of combining the outputs of multiple $\mathrm{TM}_{010}$ cavities, in support of the design of a full-scale axion detector which would either find or exclude dark matter axions in the mass range around $1 \mu \mathrm{eV}$.
\end{abstract}




\section{TABLE OF CONTENTS}

Page

I. Introduction $\ldots \ldots \ldots \ldots$

II. Background and progress report . . . . . . . . . . . . . . . . 2

A. The cavity detector of galactic halo axions . . . . . . . . . . . . . . 2

1. The detector principle . . . . . . . . . . . . . . . . 2

2. The sensitivity as a function of search rate . . . . . . . . . 4

3. The optimization of cavity design . . . . . . . . . . . . 5

B. Summary of results of the pilot experiment . . . . . . . . . . . . . . 6

1. Magnet . . . . . . . . . . . . . . . . 6 6

2. Cavities . . . . . . . . . . . . . . . . . 7

3. Microwave system . . . . . . . . . . . . . . . . . . . . . . .9

4. Spectrum analyzer . . . . . . . . . . . . . . . . . 9

5. Results . . . . . . . . . . . . . . . . . 10

C. Cavity R\&D-combining outputs . . . . . . . . . . . . . . . . . . . . . 12

D. Reports from the pilot search experiment . . . . . . . . . . . . . 15

III. Proposed work for the upcoming year _ . . . . . . . . . . . . . . . 17

A. Cavity design . . . . . . . . . . . . . . . . . . . . 17

B. Cavity fabrication . . . . . . . . . . . . . . . . . . . . . . . . . . 19

C. Cavity tuning . . . . . . . . . . . . . . . . . . . . . . . . . . . . . 19

D. Cavity synchronization . . . . . . . . . . . . . . . . . . . . . 23

E. Combining cavity outputs . . . . . . . . . . . . . . . . . . . . . . . . 24

F. Cryogenic amplifier . . . . . . . . . . . . . . . . . . . . . . . . . . . 24

References . . . . . . . . . . . . . . . . . . . 25

IV. Personnel . . . . . . . . . . . . . . . . . . . . . . . . . . . . 26

Appendix. Letter from INR Collaborators . . . . . . . . . . . . . . . . . 28 


\section{INTRODUCTION}

This proposal combines a progress report on the UF pilot axion experiment and a proposal for continued $R \& D$ towards development of a detector to search for dark matter axions in the mass range around $1 \mu \mathrm{eV}$. The detector would consist of a large magnet containing one or more microwave cavities; axions which enter the high-field region will be stimulated to decay into microwave photons when the resonant frequency of the cavity equals the mass of the axion.

Present constraints on the axion place its most likely mass in a range between $\sim 1 \mu \mathrm{eV}$ and $\sim 1 \mathrm{meV}$. The low end of this range is the most interesting, because $\sim 1 \mu \mathrm{eV}$ axions could possibly be the most abundant matter in the universe. Although such axions interact extremely weakly with ordinary matter, detectors can be built with the sensitivity to observe them, even assuming a conservative value for the axion-photon coupling strength.

The pilct experiment at the University of Florida has demonstrated the feasibility of most parts of a full-scale experiment. Extremely sensitive microwave amplifiers, with noise temperatures in the required $3 \mathrm{~K}$ range, are now available. In addition the high-Q cavities, effective tuning mechanism, low-noise amplifiers, on-line spectral analysis technique, and efficient data acquisition and control system which are necessary for the experiment have been demonstrated.

These results have eiccouraged us to begin the planning of a full-scale experiment, employing large magnets available at Lawrence Livermore Laboratory and to make detailed designs of cavities for such an experiment. The next phase of the pilot project, described in this proposal, will provide important tests of two axion detector cavity configurations. One is a modular cavity, made of plated stainless steel, which can be subdivided into two and then four segments. Cavity $Q$, cavity tuning mechanisms, and the methods for combining the output of multiple cavitys will be studied.

The second cavity configuration is the result of scientists at the Institute for Nuclear Research (INR) of the USSR having joined the axion search project. The INR plan to fabricate cavities from ceramic tubes for use in the axion delector. At least two such cavities, made in the USSR, would be built and measured as part of the research proposed here, with two scientists from the INR traveling to Gainesville for the tests. 
It is our view that the axion experiment is of high importance both for elementary particle physics and for astrophysics. In particular the recently released long-range plan "The Decade of Discovery in Astronomy and Astrophysics," commissioned by the National Research Council, ${ }^{1}$ noted the importance of the dark-matter problem in general and the axion in particular. Thus, continued development of axion detector technology is extremely well justified.

\section{BACKGROUND AND PROGRESS REPORT}

\section{A. The cavity detector of galactic halo axions}

\section{The detector principle}

About eight years ago, it was pointed out that galactic halo axions can be searched for by stimulating their conversion to photons in a strong magnetic field. ${ }^{2}$ These axions have velocities $\beta$ of order $10^{-3}$ and hence their energies

$$
\epsilon_{a}=m_{a}+\frac{1}{2} m_{a} \beta^{2}
$$

have a spread of order $10^{-6}$ above the axion mass. Consider a cylindrical electromagnetic cavity of arbitrury cross-sectional shape, permeated by a large static longitudinal magnetic field $\vec{B}_{0}$. When the frequency $\omega=2 \pi f$ of an appropriate cavity mode equals $m_{a}$, galactic halo axions can convert to quanta of excitation (photons) of that cavity mode. Only the $\mathrm{TM}_{n \ell 0}$ modes couple in the limit where the cavity is much smaller than the de Broglie wavelength $\lambda_{a}=2 \pi\left(\beta m_{a}\right)^{-1} \approx 2 \pi 10^{3} m_{a}^{-1}$ of the galactic halo axions. The power on resonance from axiun $\rightarrow$ photon conversion into the $\mathrm{TM}_{n \ell 0}$ mode is ${ }^{2,3}$

$$
\begin{aligned}
P_{n \ell}= & \left(\frac{\alpha}{\pi} g_{\gamma} \frac{1}{f_{a}}\right)^{2} V B_{0}^{2} \rho_{a} C_{n \ell} \frac{1}{m_{a}} \operatorname{Min}\left(Q_{L}, Q_{a}\right) \\
= & 3 \cdot 10^{-26} \mathrm{Watt}\left(\frac{V}{3 \mathrm{~m}^{3}}\right)\left(\frac{B_{0}}{7 \operatorname{Tesla}_{a}}\right)^{2} C_{n \ell}\left(\frac{g_{\gamma}}{0.36}\right)^{2} \\
& \cdot\left(\frac{\rho_{a}}{\frac{1}{2} \cdot 10^{-24} \mathrm{gr} / \mathrm{cm}^{3}}\right)\left(\frac{m_{a}}{2 \pi(1 \mathrm{GHz})}\right) \operatorname{Min}\left(Q_{L}, Q_{a}\right)
\end{aligned}
$$

where $V$ is the volume of the cavity, $B_{0}$ is the effective static magnetic field strength, $C_{n}$ is a mode dependent form factor, $\rho_{a}$ is the density of galactic halo axions at the earth, $Q_{L}$ is the loaded quality factor of the cavity and $Q_{a} \simeq 10^{6}$ is the "quality factor" of the galactic balo axions, i.e., the ratio of their energy to their energy spread. 
The product $B_{0}^{2} C_{n \ell}$ is deffned as follows. For $\mathrm{TM}_{n \ell 0}$ modes, one has

$$
\begin{aligned}
& \vec{E}_{\omega}=\hat{z} \psi_{n \ell}(x, y) e^{-i \omega_{n \ell} t} \\
& \vec{B}_{\omega}=\frac{i}{\omega_{n} \ell} \hat{z} \times \vec{\nabla}_{t} \psi_{n \ell}(x, y) e^{-i \omega_{n \ell} t}
\end{aligned}
$$

where $\mathrm{x}$ and $\mathrm{y}$ are the transverse coordinates and $\psi_{n \ell}$ satisfies

$$
\left(\frac{\partial^{2}}{\partial x^{2}}+\frac{\partial^{2}}{\partial y^{2}}\right) \psi_{n \ell}+\omega_{n \ell}^{2} \epsilon \psi_{n \ell}=0
$$

and the condition

$$
\left.\psi_{n \ell}\right|_{\Gamma}=0
$$

on the metallic boundaries $\Gamma$ of the cross-sectional area $S$ of the cavity, $\epsilon$ is the dielectric constant which may depend upon $\mathrm{x}$ and $\mathrm{y}$. The magnetic permeability $\mu$ was assumed to be equal to one everywhere. The product $B_{0}^{2} C_{n \ell}$ is defined by

$$
B_{0}^{2} C_{n \ell}=\frac{\left|\int_{V} d^{3} \mathrm{x} \psi_{n \ell}(\mathrm{x}, \mathrm{y}) \vec{B}_{0}(\overrightarrow{\mathrm{x}}) \cdot \hat{z}\right|^{2}}{V \int_{V} d^{3} \mathrm{x}\left|\psi_{n \ell}(\mathrm{x}, \mathrm{y})\right|^{2} \epsilon(\mathrm{x}, \mathrm{y})} .
$$

To get an idea of the mode number dependence of $B_{0}^{2} C_{n \ell}$ consider the simple case where $\vec{B}_{0}(\vec{x})$ is homogeneous and longitudinal $\vec{B}_{0}(\vec{x})=B_{0} \hat{z}$. Then, for a cavity of rectangular cross-section, one has

$$
C_{n \ell}=\left\{\begin{array}{cl}
\frac{64}{\pi^{4} n^{2} \ell^{2}} & \text { for } n \text { and } \ell \text { odd } \\
0 & \text { otherwise }
\end{array}\right.
$$

For a cavity of circular cross-section

$$
C_{n m}=\frac{4}{\left(x_{0 n}\right)^{2}} \delta_{n m}
$$

where $x_{0 n}$ is the $\mathrm{n}^{\text {th }}$ zero of the Bessel function $J_{0}(\mathrm{x})$. One sees that $C_{n \ell}$ is a sharply decreasing function of mode-number. If at all possible, one should use the fundamental TM mode. This is what we intend to do. From here on, we will suppress the indices $n, \ell$. 


\section{The sensitivity as a function of search rate}

To detect the power $P$, a hole must be made in the cavity wall through which the electromagnetic radiation can be brought to the front end of a microwave receiver. The quality factor $Q_{L}$ which appears in Eq. (2) is the loaded quality factor given by

$$
\frac{1}{Q_{L}}=\frac{1}{Q_{w}}+\frac{1}{Q_{h}}
$$

where $\frac{1}{Q_{w}}$ is the contribution due to absorption into the cavity walls and $\frac{1}{Q_{h}}$ is the contribution from the hole. The maximum power that can be brought to the front end of the microwave receiver is $\frac{Q_{L}}{Q_{h}} P$ where $P$ is given by $\mathrm{Eq}$. (2).

Because the axion mass is only known in order of magnitude at best, the cavity must be tunable and a large range of frequencies must be explored seeking the axion signal. The cavity can be tuned by inserting a dielectric rod into it, ${ }^{4}$ or by moving a dielectric rod or metal post sideways inside the cavity. ${ }^{5,6}$ Using Eq. (2), one finds that to obtain a given signal to noise ratio $s / n$, the search rate is

$$
\begin{aligned}
\frac{d f}{d t}= & \frac{55 \mathrm{GHz}}{\text { year }}\left(\frac{4}{s / n}\right)^{2}\left(\frac{V}{3 \mathrm{~m}^{3}}\right)^{2}\left(\frac{B_{0}}{7 \text { Tesla }}\right)^{4} C^{2}\left(\frac{g_{\gamma}}{0.36}\right)^{4} \\
& \cdot \frac{\rho_{a}}{\frac{1}{2} \cdot 10^{-24} \mathrm{gr} / \mathrm{cm}^{3}}\left(\frac{5 \mathrm{~K}}{T_{n}}\right)^{2}\left(\frac{f}{1 \mathrm{GHz}}\right)^{2} \cdot\left\{\begin{array}{ccc}
Q_{w} / Q_{a} & \text { if } \quad Q_{u}<3 Q_{a} \\
\frac{27}{4}\left(1-\frac{Q_{a}}{Q_{w}}\right)^{2} & \text { if } \quad Q_{u}>3 Q_{a}
\end{array}\right.
\end{aligned}
$$

where $T_{n}$ is the sum of the physical temperature of the cavity plus the system noise temperature of the microwave receiver, and where $Q_{a} \equiv 10^{6}$ now. Eq. (9) was derived assuming

1) that when $Q_{L}<Q_{a}$, i.e., when the cavity bandwidth is larger than the axion bandwidth, one uses the possibility of looking at $Q_{a} / Q_{L}$ axion bandwidths simultaneously, ${ }^{7}$

2) that $Q_{h}$ has been adjusted so as to maximize the search rate. For $Q_{w}<3 Q_{a}$, the optimal $Q_{h}=\frac{1}{2} Q_{w}$ (and hence $Q_{L}=\frac{1}{3} Q_{w}$ ) whereas for $Q_{w}>3 Q_{a}$, the optimal $Q_{h}$ occurs at $Q_{L}=Q_{a}$.

The best possible quality factors attainable at present, using high-purity oxygen free copper, are of the order of

$$
Q_{w} \simeq \frac{R}{\delta} \simeq 1.3 \cdot 10^{5}\left(\frac{\mathrm{GHz}}{f}\right)^{\frac{2}{3}}
$$

where $R$ is some measure of the transverse size of the cavity (or of the cavity cells if the cavity is divided into cells; see below) and $\delta$ is the anomalous skin depth. One has $R \sim f^{-1}$ 
and $\dot{\delta} \sim f^{-\frac{1}{3}}$. Note that on a logarithmic frequency scale the search rate to achieve a given signal to noise ratio is not sharply frequency dependent if $T_{n}$ and $C$ are not sharply frequency dependent. Indeed, by combining Eqs. (9) and (10), one finds

$$
\frac{d \ln f}{d t} \sim\left(\frac{C(f)}{T_{n}(f)}\right)^{2} f^{\frac{1}{s}} .
$$

Eq. (9) shows that a cosmic axion search sensitive to the local galactic halo density is feasible with presently available technology provided a sufficiently large magnet is availaible-such as the Axicell magnets from the MFTF-B fusion test facility at Lawrence Livermore National Laboratory (LLNL) which we are proposing to use-and provided the cavity can be designed to keep $C=O(1)$ at all frequencies of interest.

\section{The optimization of cavity design}

The problem of cavity design is the following. A large volume empty cylindrical cavity has a low resonant frequency in its lowest TM mode: $f=0.115 \mathrm{GHz}\left(\frac{1 m}{R}\right)$ where $R$ is the radius of the cavity. In terms of the physical (not angular) frequency, $1 \mathrm{GHz}$ corresponds to $4.135 \mu \mathrm{eV}$, etc. Thus a large cylindrical cavity is adsquate for searching the low end of the axion mass range of interest. The problem is: how does one search the higher frequencies? This question was addressed in a paper by C. Hagmann et al. ${ }^{5}$ which also discussed various cavity tuning schemes and presented the results of computer simulations to optimize cavity design. It was found in Ref. 5 that the best way to tune a single cylindrical cavity is by translating a dielectric rod or a metal post sideways inside the cavity. This method has been successfully tested in the pilot cosmic axion detector at the University of Florida, and it is the method we plan to use in the experiment proposed here. Secondly, Ref. 5 concluded that the best way to extend the search to high frequencies is the straightforward approach of power-combining many identical cavities which fill up the volume inside the magnet bore. This method entirely avoids the problems of mode localization and resonance crowding which plague the other approaches considered in Ref. 5. It allows one to maintain $C \simeq O(1)$ at all frequencies, albeit at the cost of increasing engineering complexity when the frequency is increased. 


\section{B. Summary of results of the pilot experiment}

At the present time two groups have conducted axion searches using cavity detectors. During 1986-1989 a collaboration between Rochester, Brookhaven, and Fermilab (RBF) carried out a search which covered energies in the $4.5-18 \mu \mathrm{eV}(1-4 \mathrm{GHz})$ range. ${ }^{4,8,9}$ In 1989-90 the Florida (UF) group scanned 5.5-7 $\mu \mathrm{eV}(1.3-1.6 \mathrm{GHz})$. The UF detector began taking data in $1989 .{ }^{10,6}$ A sketch is shown in Fig. 1.

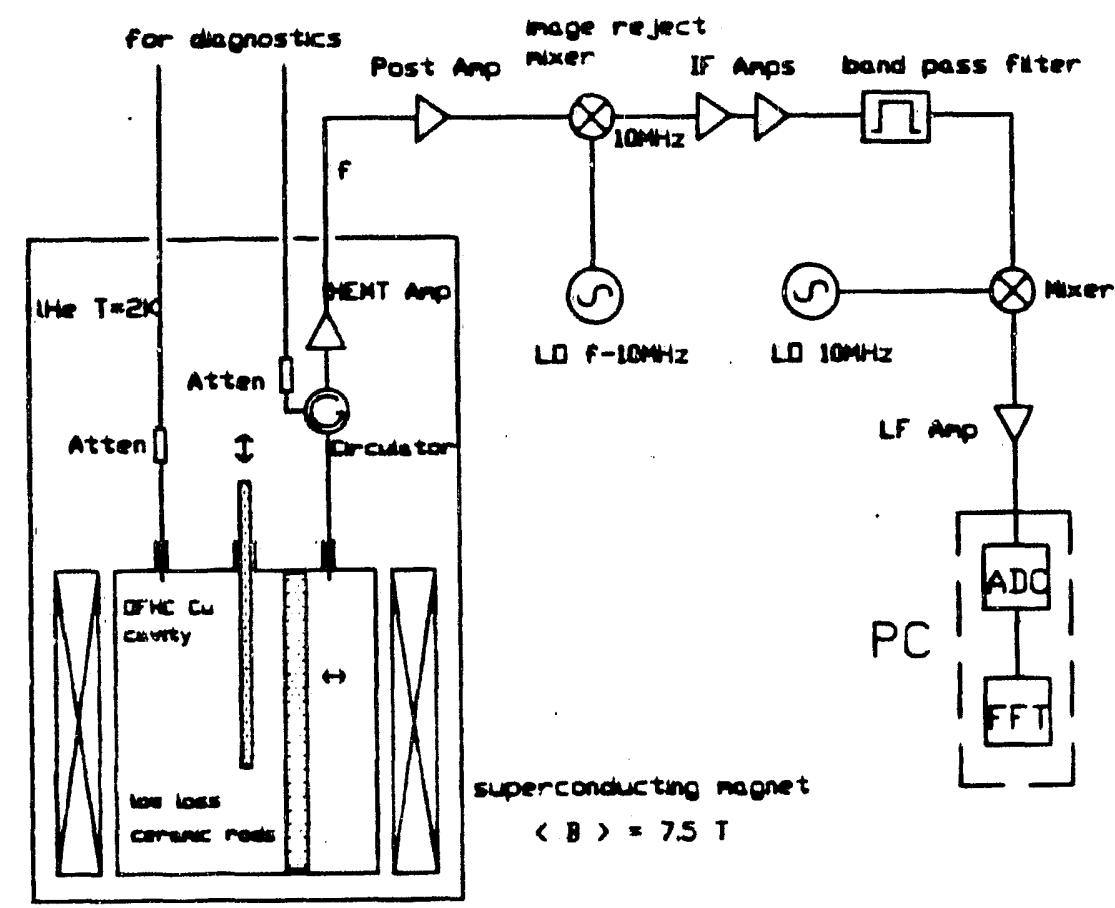

Fig. 1. Block diagram of UF axion detector, microwave circuitry, and data acquisition system

\section{Magnet}

The detector uses a superconducting magnet $40 \mathrm{~cm}$ long with $17 \mathrm{~cm}$ inner bore. The central field is $8.6 \mathrm{~T}$ while the volume-averaged field over the $0.008 \mathrm{~m}^{3}$ volume of the cavity is $7.5 \mathrm{~T}$. This gives $B_{0}^{2} V=0.45 \mathrm{~T}^{2} \mathrm{~m}^{3}$. The magnet is operated in persistent mode, typically at $2.2 \mathrm{~K}$. Temperatures below the $4.2 \mathrm{~K}$ normal boiling point of liquid helium were achieved with a " $\lambda$-fridge," in which a small stainless-steel tube containing a heat exchanger and flow impedance extends well below the surface of the helium. When the upper end of the tube is evacuated, the helium in the tube cools to approximately $2 \mathrm{~K}$ and in turn lowers the temperature in the outside bath. With this arrangement the helium around the magnet, cavity, and amplifier can be reduced in temperature while the free surface above remains at $4.2 \mathrm{~K}$, reducing superfluid film-flow losses and permitting retransfer of the helium without warming the magnet. 


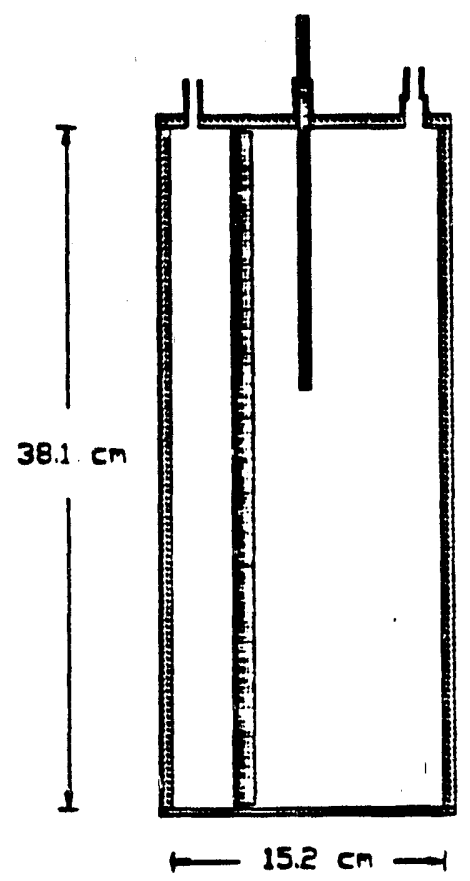

Fig. 2. Cavity with two dielectric tuning rods. The larger rod is translated sideways; the smaller one along the cylinder axis. Two coupling ports are also shown.

\section{Cavities}

Two cavities were used, differing by $10 \%$ in diameter. The cavities, made from OFHC copper and electropolished to provide good surfaces, were operated in the $\mathrm{TM}_{010}$ mode. Fig. 2 shows the cavity along with tuning rods and coupling ports. Unloaded quality factors were measured to be $Q_{w} \approx 150,000$, and nearly $T$-independent below $20 \mathrm{~K}$. The wall quality factor for the $\mathrm{TM}_{010}$ mode is

$$
Q_{w}=\frac{L}{L+R} \frac{R}{\delta}
$$

where $L$ is the length and $R$ the radius of the cavity; the skin depth $\delta$ in the extreme anomalous skin effect regime is $\delta=\left(\sqrt{3} c^{2} m_{e} v_{F} / 8 \pi^{2} \omega N_{e} e^{2}\right)^{1 / 3} \approx 0.28 \mu \mathrm{m}$. Here, $m_{e}$ is the electron mass, $v_{F}$ the Fermi velocity, and $N_{e}$ the conduction electron density. For the parameters of the cavities used, Eq. 12 predicts $Q_{w}^{A S E}=250,000$, about $60 \%$ larger than what was measured. The discrepancy may be due to parasitic losses at the coupling port, to a small build-up of oxide, and to scratches and deformation of the cavity walls.

The larger of the two coupling ports-the major port-is overcoupled and connected to the amplifier. It consists of an inductive loop at the end of a coaxial cable. Springloaded $\mathrm{BeCu}$ fingers make electrical connection between the outer sheath of the coax and the cavity, allowing the coupling strength of the cavity to be varied by either changing the 
insertion depth of the loop or by rotating the loop. We found that either coupling method worked well, allowing $Q_{h}$ to be varied from $2 \times$ overcoupled to substantially undercoupled without affecting other cavity parameters. The smaller coupling port in Fig. 2 is used for measuring the resonance frequency and quality factor in transmission mode, as well as for other diagnostic measurements.

The cavity is tuned by moving dielectric or metal rods located in the cavity. Two tuning rods are employed: coarse tuning is achieved with a larger rod-either dielectric or metalthat is moved radially while fine tuning uses a small dielectric rod inserted along the cavity axis. The purpose of using two tuning rods is to enable rather large tuning ranges $( \pm 20 \%)$ while avoiding problems of longitudinal mode localization, ${ }^{5}$ which degrades the cavity form factor $C$. The difficulties associated with localization were discovered during the course of the pilot project, and the two-rod tuning was developed to overcome these difficulties. We plan to eliminate the axial rod completely in the next cavity design.

As the cavity is tuned there are a number of mode crossings between the $\mathrm{TM}_{010}$ mode and various TE modes. Because of unavoidable mixing of the two modes in this experiment there is a region a few hundred $\mathrm{kHz}$ wide at each crossing where the detector does not work. These holes comprise less than $0.2 \%$ of the scanned spectrum. Fig. 3 shows one such crossing in Cavity I. This figure, which shows the resonant frequency as a function of the insertion depth of the small tuning rod, also illustrates the precision of cavity tuning. A travel of $1 \mathrm{~cm}$ leads to a $1 \mathrm{MHz}$ change in the $\mathrm{TM}_{010}$ frequency. The smallest step is $\approx 4$ $\mu \mathrm{m}$, which changes the frequency by about $400 \mathrm{~Hz}$.

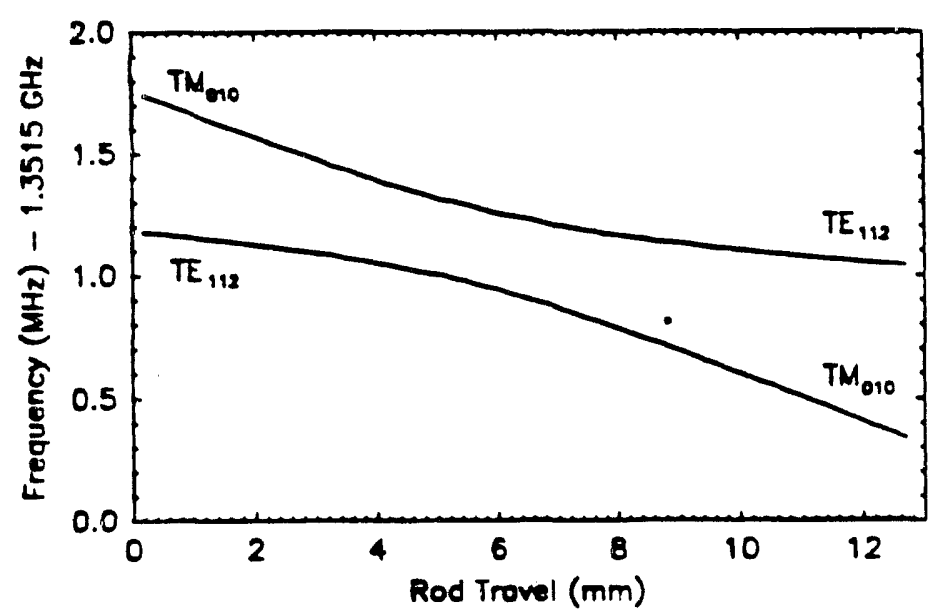

Fig. 3. Measured mode crossing between the $\mathrm{TE}_{112}$ and $\mathrm{TM}_{010}$ cavity modes. The ordinate is the insertion depth of the small tuning rod along the cavity axis. 


\section{Microwave system}

The cryogenic microwave amplifiers were purchased from Berkshire Technologies. ${ }^{11}$ These are three-stage GaAs amplifiers, with the first stage device being a high electron mobility transistor (HEMT). The amplifiers work at all temperatures between $300 \mathrm{~K}$ and 2 $\mathrm{K}$, providing about $35 \mathrm{~dB}$ power gain, with noise temperatures in the $3-5 \mathrm{~K}$ range at the lowest temperature.

Magnetically shielded cryogenic circulators ${ }^{12}$ are inserted between the cavity and amplifier input terminal to permit the cavity to be isolated from the amplifier. The third arm of the circulator is then used to bring microwave power to the major port, so that its reflection coefficient can be measured and coupling adjusted. A $50 \mathrm{~dB}$ attenuator in the helium bath kept room temperature noise from entering the cavity.

The remainder of the experimental setup in Fig. 1 is the double-superheterodyne receiver, the digital spectrum analyzer, and the computer control system. The output of the cryogenic amplifier is further amplified $(50 \mathrm{~dB})$ by a $300 \mathrm{~K}$ microwave amplifier and converted to the $10.7 \mathrm{MHz}$ first IF frequency by an image reject mixer. This mixer, assembled from commercially available components, used a synthesizer as local oscillator. The rejection of the unwanted sideband was $\geq 18 \mathrm{~dB}$ and averaged $22 \mathrm{~dB}$.

The IF amplifiers have a combined gain of $60 \mathrm{~dB}$; they are followed by an 8-pole crystal filter, which determines the bandwidth of the system. The filter response is reasonably flat over its $30 \mathrm{kHz}$ passband, with over $50 \mathrm{db}$ rejection of out-of-band signals. This filter has a significant temperature variation to its response. It was therefore necessary to enclose it in a temperature-stabilized enclosure, which reduced drift to an acceptable level. The second converter uses a double-balanced mixer and $10.67 \mathrm{MHz}$ local oscillator. This provides an audio signal spanning $0-30 \mathrm{kHz}$ which undergoes a final amplification before $\mathrm{A} / \mathrm{D}$ conversion.

\section{Spectrum analyzer}

We use a digital fast Fourier transform (FFT) scheme to spectrum analyze the signal from the cavity. The analyzer consists of a 16 bit $A / D$ converter coupled with a digital signal processor. The audio signal (bandwidth $30 \mathrm{kHz}$ ) is sampled at a $70 \mathrm{kHz}$ rate, with a total of 64 points measured. These data are Fourier transformed and the power spectrum calculated, giving a resolution of $\approx 1 \mathrm{kHz}$, the width of the axion signal when $m_{a}=1 \mathrm{GHz}$. Successive spectra are averaged to obtain an estimate of the power emitted from the cavity over its bandwidth.

The signal processing board ${ }^{13}$ consists of the A/D converter, a $40 \mathrm{MHz}$ signal processing chip, ${ }^{14}$ and $64 \mathrm{~kb}$ of memory, all located in the 80386 -based $\mathrm{PC}$ which serves as the system 
controller. The board is fast enough to perform the FFT, calculate the power spectrum, and make the average in just under $0.6 \mathrm{~ms}$. Because each 64-point scan is acquired while the previous scan is being Fourier analyzed and averaged, the analyzer has a high duty factor.

The time for a single A/D scan is $0.9 \mathrm{~ms}$; a total of $10^{5}$ spectra are acquired and averaged in $90 \mathrm{sec}$. Immediately afterwards, each average spectrum is searched for $2 \sigma$ peaks in single bins and combination of neighboring bins. If a peak is found, another set of $10^{5}$ spectra is taken and averaged with the first. If the peak remains statistically significant this process is repeated up to a maximum of 5 times, after which the peak is flagged for later investigation. This repetition improves the signal/noise ratio of candidate signals by just over a factor of two.

To continue the run the computer writes the completed spectrum to disk, steps the small tuning rod, introduces a frequency-swept test signal into the cavity minor port, measures the cavity $Q$ and resonant frequency, and instructs the signal processing board to get the next set of spectra.

Fig. 4 shows a typical spectrum, with no peaks exceeding the $2 \sigma$ level. Fig. 5 shows one of several peaks which survived the test described above. When re-examined with the magnetic field $B_{0}$ off, all of these peaks persisted, and therefore were not signals coming from axion conversion. Instead they were from external sources, in particular from the computer and other digital equipment in the vicinity of the experiment.

\section{Results}

To date three regions have been investigated, with a total operating time of a little over five months. Two separate runs were made over 1.32-1.44 GHz, one at bath temperature $T=2.2 \mathrm{~K}$ and the other at $T=4.2 \mathrm{~K}$, and their statistics combined. A single run was made over $1.44-1.60 \mathrm{GHz}$ at $2.2 \mathrm{~K}$. Another short run was made over a narrow region near $1.8 \mathrm{GHz}$. The experiment ${ }^{15}$ is thus able to set the upper limit on $g_{a \gamma \gamma}^{2}$ (for $\rho_{a}=\rho_{\text {halo }}$ ) plotted in Fig. 6. Also shown is the limit obtained by the RBF group. ${ }^{4,8,9}$

The sensitivity of the detector is set by thermal noise. The noise power fluctuation is given by

$$
\Delta P_{n}=k_{B} T_{n} \mathcal{B} / \sqrt{N_{a v}}
$$

where

$$
T_{n}=T_{a m p}+T_{b a t h}
$$

is the system noise temperature, $\mathcal{B}$ is the $1 \mathrm{kHz}$ bin width and $N_{a v}$ is the number of averages. 


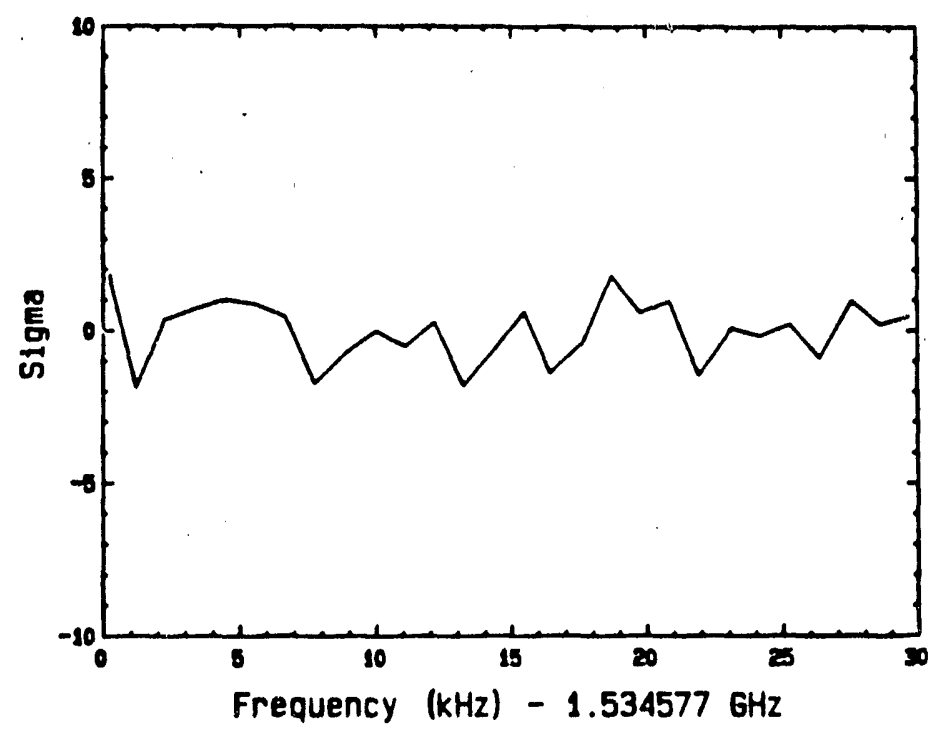

Fig. 4. Typical power spectrum.

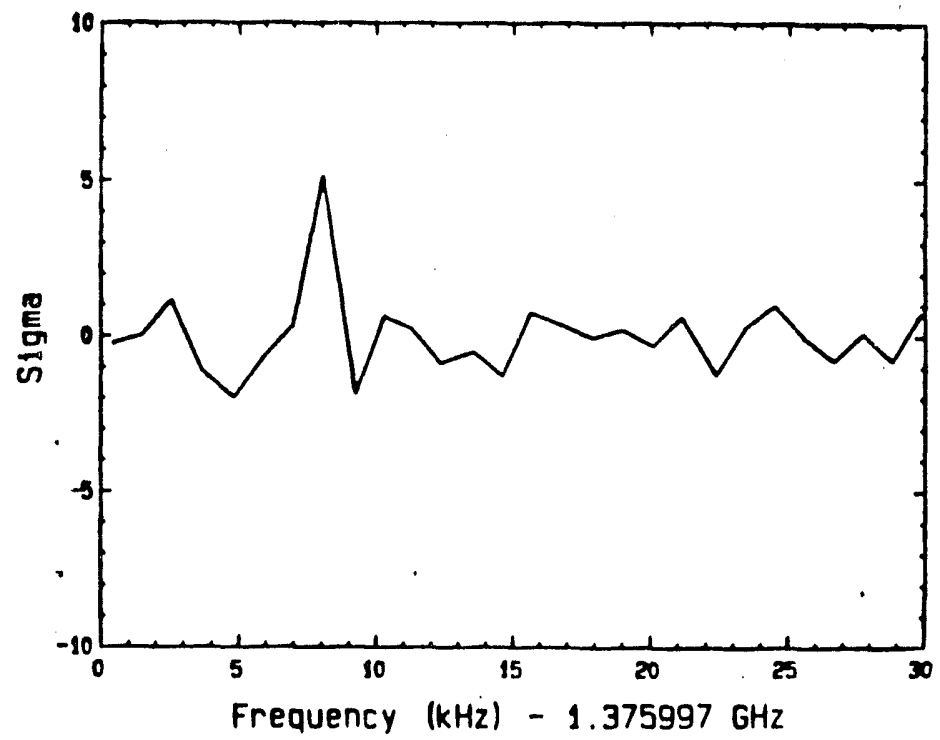

Fig. 5. Power spectrum with candidate peak.

These fluctuations set our minimum detectable signal power (at the 95\% C.L.) to be

$$
P_{\text {signal }} \simeq 8 \times 10^{-22} \mathrm{Watt}
$$

for $\mathcal{B}=1100 \mathrm{~Hz}(1 \mathrm{bin})$ and $\sqrt{2}$ times that for a signal falling into two bins. On account of the modest volume of the cavity, this noise power level is still a factor of $\sim 500$ too large for the signal expected from axion conversions, $P_{a \rightarrow \gamma} \simeq 1.3 \times 10^{-24}$ Watt in the DFSZ model assuming $\rho_{a}=\rho_{\text {halo }}$. 


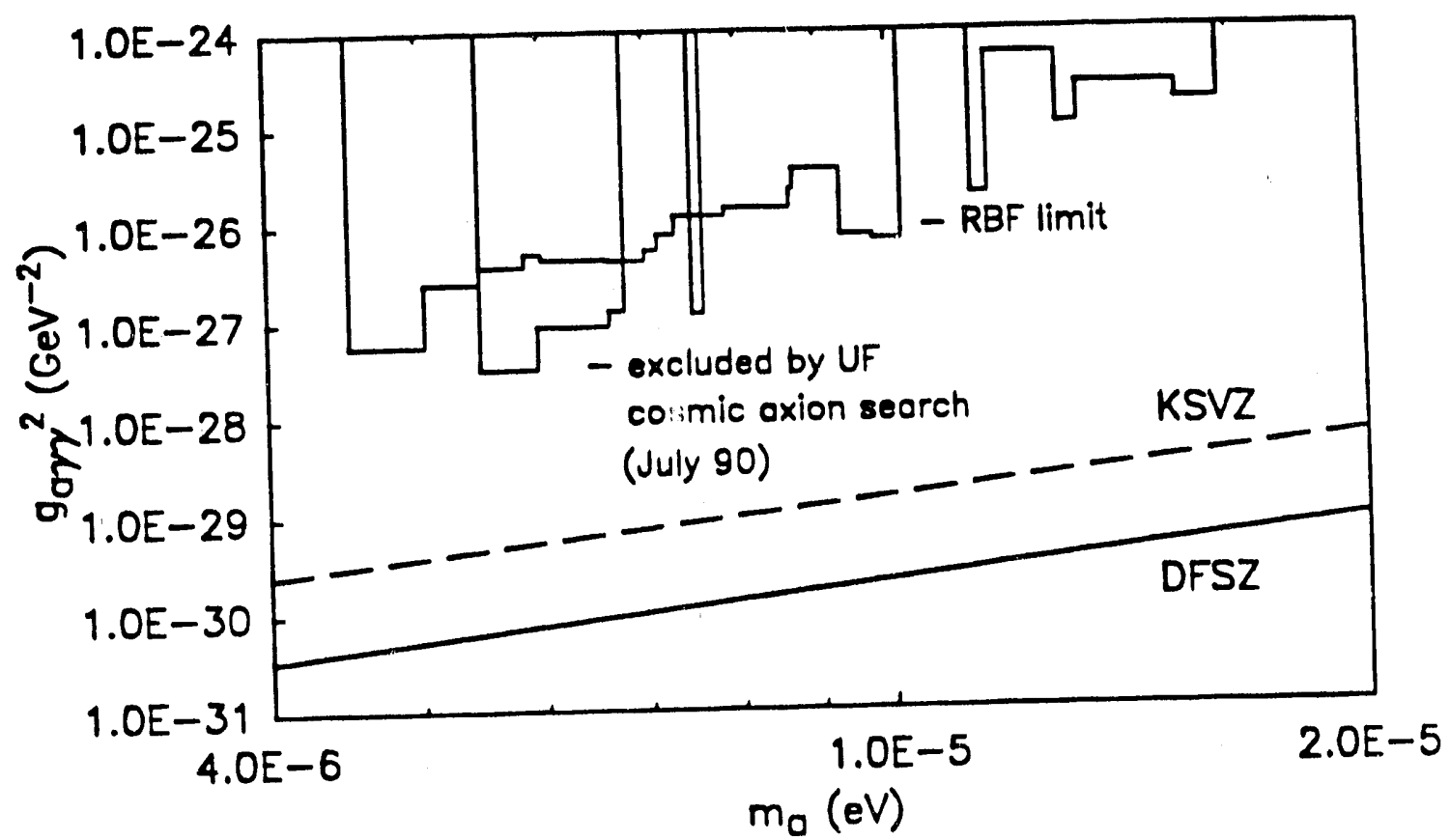

Fig. 6. Experimental limit obtained by the UF experiment on axion ccuplings $g_{a \gamma \gamma}^{2}$ assuming $\rho_{a}=\rho_{\text {halo }}=5 \times 10^{-25} \mathrm{~g} / \mathrm{cm}^{3}$.

\section{Cavity R\&D-combining outputs}

In the multi-cavity detector, the outputs of all the cavities need to be brought in phase to the inputiof the microwave amplifier. This means that

1. all the cavities must have the same resonant frequency,

2. the coupling $Q$ (i.e., $Q_{h}$ ) must be the same for all the cavities, and

3. the length of RF cable between each cavity and the power combiner must be the same. Power splitters/combiners are used to combine the signals into a single coaxial line. These passive devices transmit all of the power incident on them when the individual input signals have equal amplitude and phase. Otherwise, some of the incident power is lost in a termination.

Although this appears a difficult task, we have made a preliminary demonstration for the simplest case of two cavities. The test setup is shown in Fig. 7. The empty cavities had $\mathrm{TM}_{010}$ resonance frequencies of $1.67 \mathrm{GHz}$ with $Q_{\text {wall }} \sim 3 \times 10^{4}$. The frequencies differed initially from each other by $0.7 \%$, but could be made to match by inserting a small Teflon tuning rod $(0.4 \mathrm{~cm}$ in diameter $)$ into one of the cavities.

Two identical Wilkinson power dividers/combiners ${ }^{16}$ were used to split and combine 


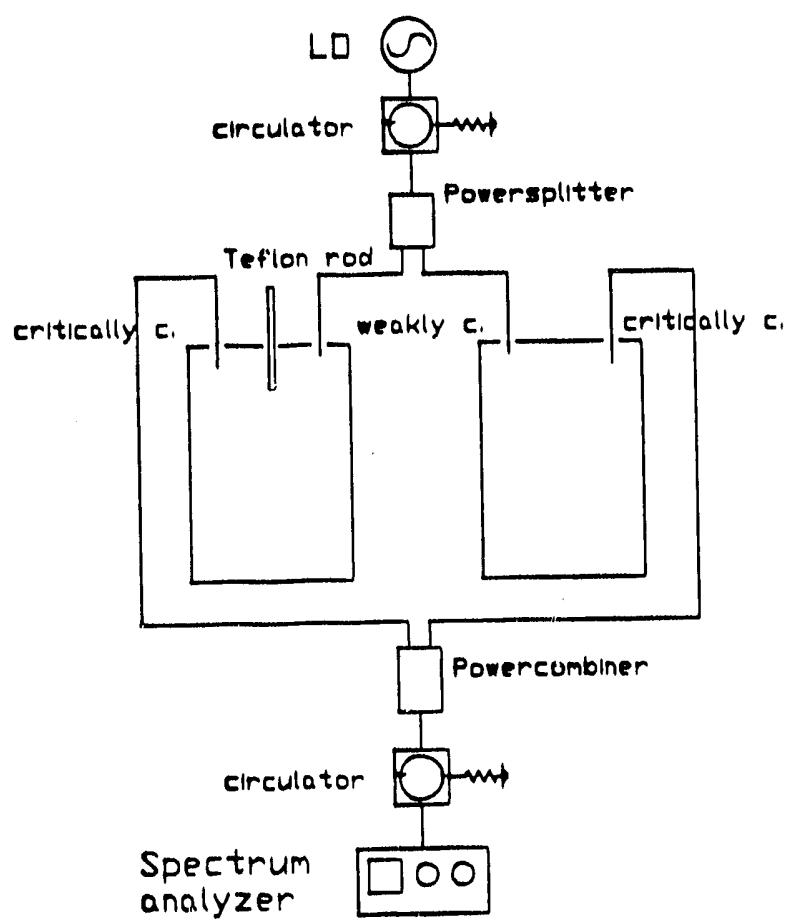

Fig. 7. Test setup of two frequency-matched cavities.

the signals in phase. A signal of voltage $V \sim e^{-i \omega t}$ entering the divider is split into equal amplitude signals $V \sim e^{-i \omega t} / \sqrt{2}$ at the output. When used as a combiner with amplitudes $a e^{-i \omega t}$ and $b e^{-i \omega t-i \phi}$, the output voltage is $\left(a+b e^{-i \phi}\right) e^{-i \omega t} / \sqrt{2}$ and power is dissipated in internal resistors unless the two inputs are exactly balanced.

In the experiment, one of the ports of each cavity was critically coupled, while the other was weakly coupled. The cavity can be modeled by a series $R L C$-circuit with resonance frequency $\omega_{0}^{2}=1 / L C$ and $Q_{u}=\omega_{0} L / R$. The input impedance of a single port cavity near resonance is given by

$$
Z_{c}=\frac{Z_{0}}{\beta R}\left(R+i \omega L+\frac{1}{i \omega C}\right) \simeq \frac{Z_{0}}{\beta}\left(2 i Q_{w} \frac{\omega-\omega_{0}}{\omega_{0}}+1\right)
$$

where $\beta=Q_{w} / Q_{h}$ is the coupling parameter and $Z_{0}$ is the characteristic line impedance, usually $50 \Omega$. The input impedance is equal to the line impedance for a critically coupled cavity $(\beta=1)$ on resonance, making the cavity reflectionless. For a two port cavity, Eq. (16) still applies but $R$ is modified to include the additional losses from the second port. A signal transmitted through a critically coupled cavity (while the second port is weakly coupled) will suffer a phase shift $V \sim e^{i \alpha}$ with

$$
\tan \alpha=\left(\frac{1}{\omega C}-\omega L\right) / 2 R \simeq 2 Q_{L} \frac{\omega-\omega_{0}}{\omega_{0}} \equiv x
$$


and the amplitude is decreased by a factor $1 / \sqrt{1+x^{2}}$ relative to the value on resonance.

The output voltage of the power combiner for the LO frequency at the resonance frequency of the first cavity and variable resonance frequency of the second cavity is then

$$
V \cdot=\frac{V_{0}}{\sqrt{2}}\left(\frac{1}{\sqrt{2}}+\frac{1}{\sqrt{2}} \frac{1}{\sqrt{1+x^{2}}} e^{i \alpha}\right)
$$

and the power

$$
\left|\frac{V}{V_{0}}\right|^{2}=\frac{1}{4}\left(1+\frac{3}{1+x^{2}}\right)
$$

This relationship is plotted in Fig. 8. Also shown are the measured values which are in good thgreement with the theoretical curve. Operation of a multi-cavity detector would require keeping the cavity resonance frequencies within a few percent of the cavity width in order to avoid mismatch losses. This test demonstrates the feasibility of using phased multi-cell cavities as axion cavity detectors.

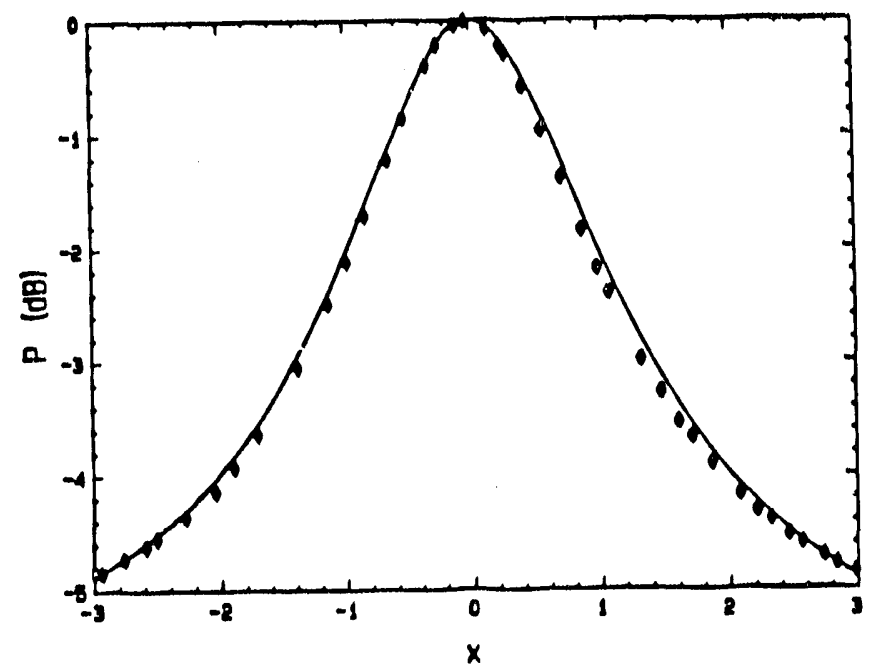

Fig. 8. Transmitted power for the configuration shown in Fig. 7.

A direct coupling of the cavities with mode mixing and splitting into symmetric and antisymmetric modes was not observed in this arrangement, on account of the large isolation ( $>30 \mathrm{~dB}$ ) between the power combiner arms. Mixing was observed, however, when the strongly coupled ports were directly connected together.

The calculation agrees with the measurement within $\pm 0.2 \mathrm{~dB}$, with the difference largely due to the uncertainty in determining the variable resonance frequency of the second cavity. Note that when the two cavities were synchronized, the calculation and measurement agree extremely well. (A correction has been made for the $0.1-0.2 \mathrm{~dB}$ loss in the power combiner.) 


\section{Reports from the pilot search experiment}

The UF axion experiment has produced a number of papers and presentations. These are listed in this section.

\section{Papers}

1. "Microwave Detector for Galactic Halo Axions," S.-I. Cho, C. Hagmann, P. Sikivie, N.S. Sullivan, and D.B. Tanner, Jpn. J. Appl. Phys, 26, 1705-1706 (1987).

2. "Cavity design for a cosmic axion detector," C. Hagmann, P. Sikivie, N.S. Sullivan, and D.B. Tanner, in Proceedings of the Storrs Meeting, edited by K. Haller et al. (World Scientific, Singapore, 1989), pp. 249-251.

3. "A cosmic axion detector," C. Hagmann, P. Sikivie, N.S. Sullivan, and D.B. Tanner, in Quantum Fluids and Solids-1989 edited by Gary G. Ihas and Yasumasa Takano (American Institute of Physics, New York, 1989), pp. 366-367.

4. "Experience with the Florida cosmic axion detector," C. Hagmann, P. Sikivie, N.S. Sullivan, and D.B. Tanner, in Proceedings of the Workshop on Cosmic Axions, edited by C. Jones and A. Melissinos (World Scientific, Singapore, 1990), pp 42-57.

5. "Cavity design for a cosmic axion detector," C. Hagmann, P. Sikivie, N. Sullivan, D.B. Tanner, and S.-1. Cho, Rev. Sci. Instrum. 61, 1076-1085 (1990).

6. "Results from a search for cosmic axions," C. Hagmann, P. Sikivie, N.S. Sullivan, and. D.B. Tanner, Phys, Rev. D 42, 1297-1300 (1990).

7. "A cosmic axion experiment," C. Hagmann, P. Sikivie, N.S. Sullivan, and D.B. Tanner," in Proceedings of the UCLA Particle Astrophysics Conference edited by Z. Cline et al.. (1991).

8. "A proposed search for dark matter axions in the 0.6-16 $\mu \mathrm{eV}$ range," Karl van Bibber, P. Sikivie, N.S. Sullivan, D.B. Tanner, D. Moltz, and M. Turner," in Proceedings of the UCLA Particle Astrophysics Conference edited by Z. Cline et al.. (1991).

9. "A proposed search for dark matter axions in the $0.6-16 \mu \mathrm{eV}$ range," C. Hagmann, M.S. Turner, P. Sikivie, N.S. Sullivan, D.B. Tanner, D.M. Moltz, P..Anthony, K. van Bibber, J.R. Miller, R. Patrick, S. Shen, and D. Slack, in Proceedings of the Santa Fe. IEEE Meeting.

\section{Presentations}

1. "Dielectric properties of low-loss materials at low temperatures," C.A. Hagmann, * Shin-Il Cho, P. Sikivie, N.S. Sullivan, and D.B. Tanner, Bull. Am. Phys. Soc, 32, 922 (1987). (New York)

2. "Axion Haloscope," S.I. Cho, C. Hagmann, P. Sikivie, N.S. Sullivan, and D.B. Tanner, Workshops on Non-Accelerator Particle Physics (Rochester, 2 June 1987). (Poster)

3. "Microwave detector for galactic halo axions," S.-I. Cho, ${ }^{*}$ C. Hagmann, P. Sikivie, N.S. Sullivan, and D.B. Tanner, International Conference on Low Temperature Physics (LT-19) (Kyoto, August 1987). (Poster) 
4. "Experience with the University of Florida Detector," C. Hagmann," P. Sikivie, N.S. Sullivan, and D.B. Tanner, Workshop on Cosmic Axions (Brookhaven, 13 April 1989). (Invited)

5. "A cosmic axion detector", C. Hagmann, P. Sikivie, N.S. Sullivan, and D.B. Tanner, Symposium on Quantum Fluids and Solids-1989 (Gainesville, 25 April 1989). (Poster)

6. "Experimental limits on the abundance of cosmic axions," C. Hagmann, ${ }^{*}$ P. Sikivie, N.S. Sullivan, and D.B. Tanner, Bull. Am. Phys. Soc. 35, 1467 (1990). (Washington)

7. "A proposed search for dark matter axions in the 0.6-16 $\mu \mathrm{eV}$ range," C. Hagmann, M.S. Turner, P. Sikivie, N.S. Sullivan, D.B. Tanner, D.M. Moltz, P..Anthony, K. van Bibber, J.R. Miller, R. Patrick, S. Shen, and D. Slack, IEEE Nuclear S'cience/Nuclear Power Symposium Santa Fe, 5 November 1991. 


\section{PROPOSED WORK FOR THE UPCOMING YEAR}

We believe that continued $R \& D$ is vitally important as we plan for a comprehensive axion search. The full-scale experiment would use two large coils from the MFTF-B fusion reactor at LLNL to provide a field of $7 \mathrm{~T}$ in a space $1.25 \mathrm{~m}$ in diameter and $2.2 \mathrm{~m}$ in length giving a volume of $2.8 \mathrm{~m}^{3}$. The quantity $B_{0}^{2} V$, which governs the sensitivity of the detector, is thus a factor of 3000 times larger than in the pilot experiment. It is this large increase in volume which allows the full-scale experiment either to find or to exclude (at the $95 \%$ C.L.) dark matter axions in the mass range around $1 \mu \mathrm{eV}$.

It is crucial to use the magnetic volume efficiently as the search is extended above the $180 \mathrm{MHz}$ fundamental frequency of a single cylindrical cavity. This section describes R\&D on cavity arrays and receiver for the experiment.

\section{A. Cavity design}

In order to understand the proposed design for the multiple-cavity arrays, some general constraints and practical considerations must be introduced. First, the $\mathrm{TM}_{010}$ mode is the only feasible one in which to operate this experiment. In this mode, the electric field $\vec{E}(x, y)$ is translationally invariant and is directed along the $\hat{z}$-axis of the cylindrical cavity, i.e., $\vec{E}=E_{z}(x, y) \hat{z}$. As the external magnetic field $\vec{B}_{0}$ is also in the $\hat{z}$ direction, the form factor $\mathrm{C}$ for a right circular cylindrical cavity in the $\mathrm{TM} 010$ mode is by far the largest of all the modes, $C=0.69$.

Second, searching for axions in a higher mass range implies correspondingly higher frequencies, and thus cavities of smaller radii. Because the signal power for axion $\rightarrow$ photon conversion goes like $B_{z}^{2} V$, where $V$ is the volume of the cavity or cavities, it is essential to close-pack as many cavities of the size appropriate to the mass range being searched into the available volume. Each cavity would be tuned independently to the same frequency, and their outputs combined and brought in phase to the front end of the low-noise amplifier. As the power splitters/combiners used to add up the outputs of the individual cavities involve combining $2^{n}$ signals into one, we have designed our cavity arrays in powers of 2.

Third, a given cavity may be tuned by approximately $\pm 25 \%$ without serious degradation of its $C^{2} Q$ by radial displacement of metal or dielectric tuning rods within the cavity. (See discussion of cavity tuning below.) With these considerations, it is possible to scan nearly a factor of 10 in frequency (mass) with a minimal number of distinct cavity arrays (6).

The magnet is initally filled with a single cylindrical cavity which has a $\mathrm{TM}_{010}$ resonant frequency of $f_{0}=115 / R \mathrm{MHz}-\mathrm{m}$. Next, a partition will be used to divide the cavity into 
two cavities, each of semicircular cross section. Each half has a $\mathrm{TM}_{010}$ resonant frequency of $1.56 f_{0}$. The fleld pettern for such a divided cavity is shown in Fig. 9.

Four cavities can be obtained by further subdividing the cylinder into quarter-circular sections. The field pattern for this configuration, which resonates at $2.1 f_{0}$, is shown in Fig. 10. Note that both the two- and four-cavity arrangement use almost all of the magnet volume.

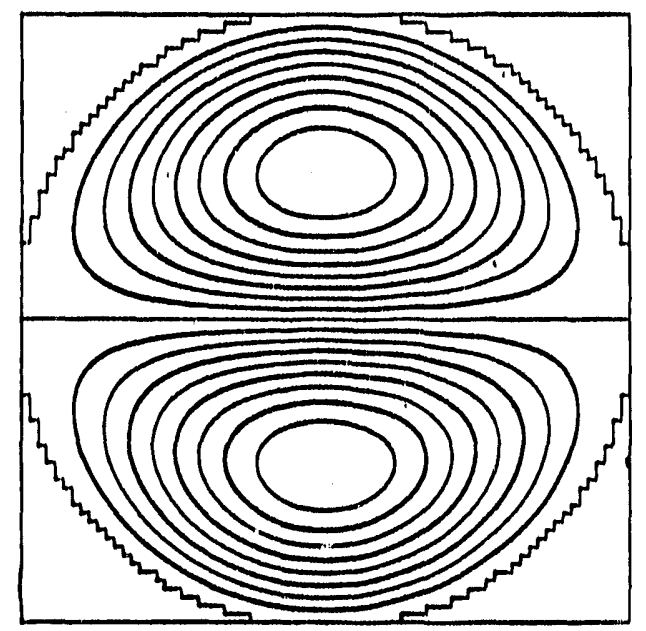

Fig. 9. Field pattern for a pair of cavities with semi-circular cross section.

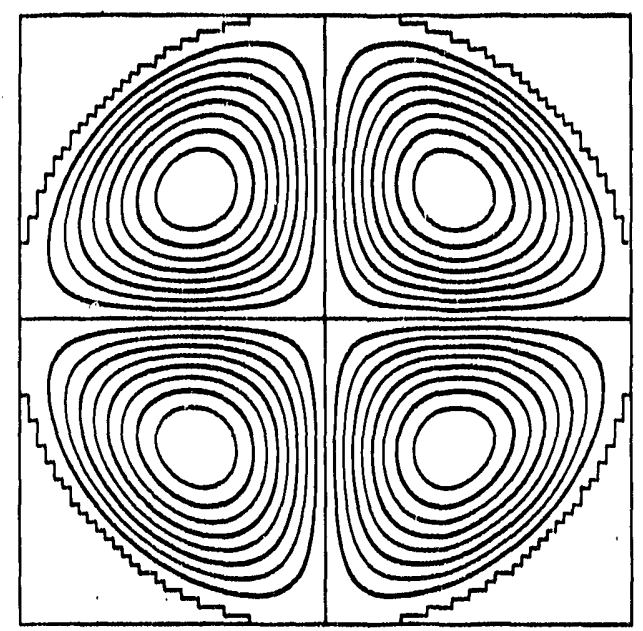

Fig. 10. Field pattern for four cavities with quarter-circular cross section. 


\section{B. Cavity fabrication}

Our pilot experiment (as well as the RBF experiment) utilized small single cavities, which were made of oxygen-free high-conductivity (OFHC) copper. The large-scale experiment will require several arrays to cover the proposed frequency range, each array typically consisting of multiple cavities considerably larger than those used in the pilot experiments. Fabricating the cavities for the large-scale experiment from OFHC tube stock then may not be optimal in view of cost, weight and rigidity.

We have arrived at a tentative cavity design for the first-three cavity arrays in which the cavities are assembled from modular quarter-sections of rolled stainless steel electroplated with copper. Fig. 11 shows a schematic end view, demonstrating how the quarter-sections from a single circular-cross sectional cylinder are reused in the semi-circular and quartercircular cavities, by replacing the spar(s) with partitions.

We propose to construct such a modular series of cavities with $f_{0} \approx 1.5 \mathrm{GHz}$. These would then be operated as part of the pilot project at UF. During the past year we have made tests of a prototype $3 \mathrm{GHz}$ copper-plated stainless-steel cavity. We found $Q \approx 40000$, a value about a factor of 2 smaller than the $Q$ we achieved using OFHC copper tubing. Thus, we will need to work to improve welding and plating techniques. In addition, we plan tests of silver and gold-plated cavities.

Our INR collaborators have proposed an examination of electroplated cavities made of precision-machined ceramic. The INR, will provide the cavities for testing during the next year, with two INR physicists expected to visit Florida during the spring to participate in the cavity tests. The details of the INR cavity design are given in Appendix A.

\section{Cavity tuning}

The cavities will be tuned by moving either a dielectric rod or metal rod radially inwards from the outer wall to the center. With either rod located near the wall, the frequency is close to that of an empty cavity. The dielectric rod gives a decrease in resonant frequency as it moves to the center; the metal rod an increase. Our simulations ${ }^{5}$ and measurements ${ }^{6}$ indicate that roughly a $20 \%$ decrease and $30 \%$ increase from the empty cavity resonance can be achieved without too serious a loss in $C^{2} Q$. Figs. 12 and 13 show tuning with sideways motion of a dielectric and a metal rod respectively. The same cavity was used in both cases; the empty resonant frequency was $1.5 \mathrm{GHz}$.

Our specific implementation of the radial translation tuning scheme has two important features. The first is mounting the rods on circular rotor-disks which are part of the cavity end-walls. The diameter of the rotor-disks is such that a tuning rod may be brought from 


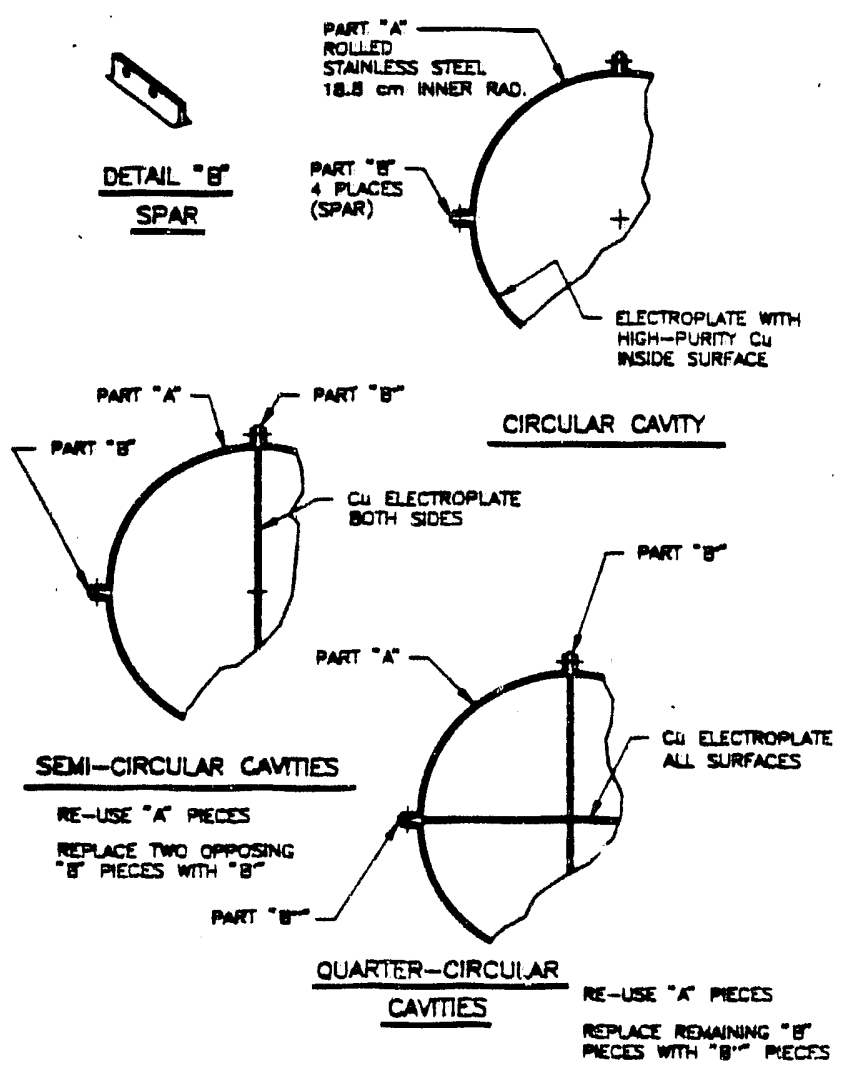

Fig. 11. Schematic end-view of circular, semi-circular and quarter-circular cross-sectional cavities, showing the modular construction based on the plated rolled stainless steel body sections.

the wall to the center of the cavity with a half-turn of the rotor-disk. This construction preserves a smooth inner wall to the chamber and is the simplest way to convert the rotary motion from the stepping motors to a radial translation. The two opposing rotor-disks are driven together by a rigid rod external to the cavity body. This means that we do not risk putting a torque (and thus twisting) the turning rods themselves by attempting to drive them from one end alone. The second feature is that each cavity has two independent tuning rods permanently mounted inside, one metal and one dielectric. With both rods positioned next to the wall, the frequency of the cavity will be approximately that of an empty cavity (as the electri. field goes to zero at the wall, so does the perturbation due to a tuning rod at that position). One tuning rod may then be stepped in towards the center, leaving the other at the wall. This arrangement has three important advantages. (i) There is no gap in coverage at the central (empty-cavity) frequency, that would result from having only one rod 


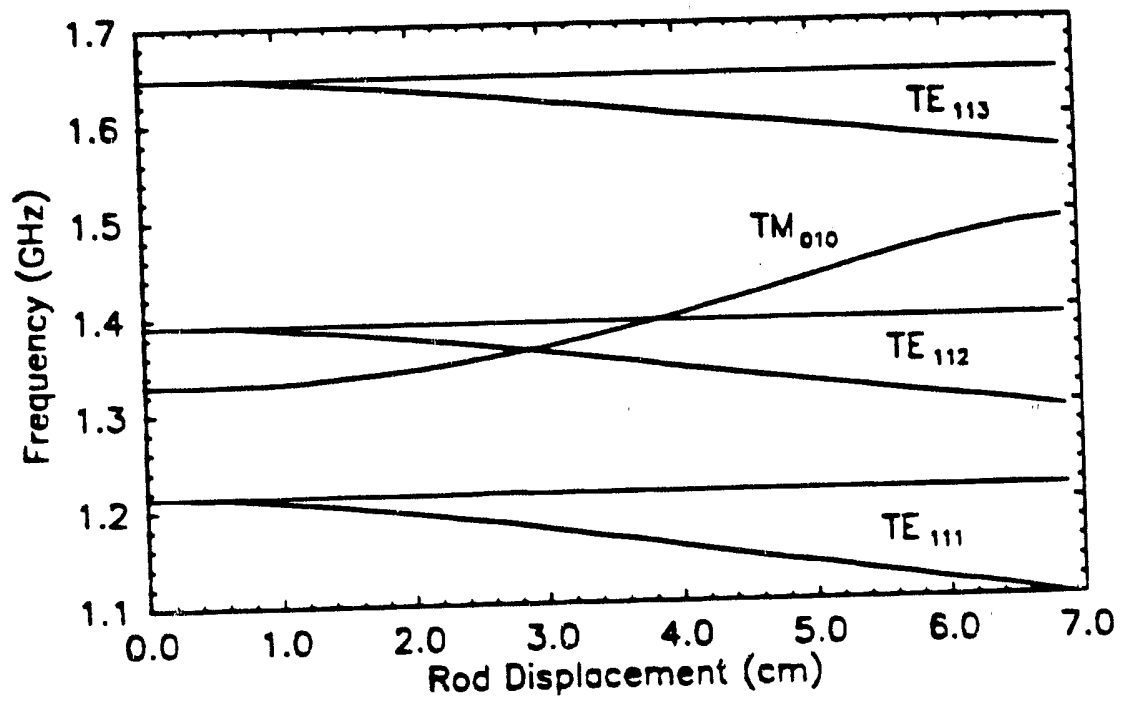

Fig. 12. Cavity frequency versus radial displacement of a dielectric rod. $(R=7.62 \mathrm{~cm}, r=0.635$ $\mathrm{cm}$, and $\epsilon=9.5$.) The $\mathrm{TM}_{010}$ mode used in the search is shown, along with nearby $T E_{11 \ell}$ modes. Note the lifting of the degeneracy of the $T E_{11 \ell}$ modes as the rod is moved sideways.

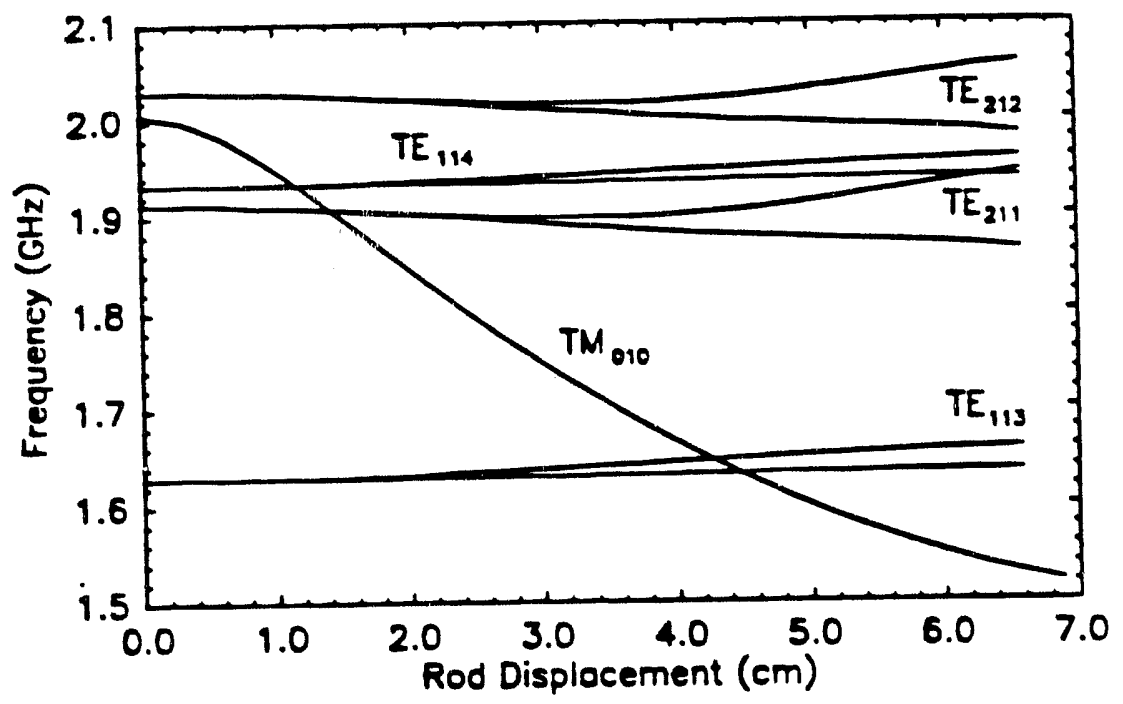

Fig. 13. Cavity frequency versus radial displacement of a metal rod. $(R=7.62 \mathrm{~cm}$ and $r=0.622$ $\mathrm{cm}$.) The $\mathrm{TM}_{010}$ mode used in the search is shown, along with nearby TE modes. 
in the cavity at a time. (ii) Half of the down-time that would be associated with warming up, removing and disassembling the cavity arrays to change from metal to dielectric iuning rods are eliminated. (iii) The small gaps in tuning range that would normally be low due to TE-TM mode-crossing are avoided, because any particular $\mathrm{TM}_{010}$ frequency may now be reached by a range of dielectric and metal rod positions-which have entirely different $T E_{n \ell m}$ frequencies. This degree of freedom will thus allow us to side-step the discrete number of mode-crossings which one would have ordinarily encountered. Figs 14 and 15 schematically represent the arrangement and mechanical assembly of the tuning assembly.

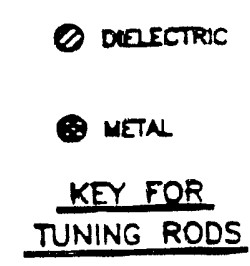

TUNING RODS

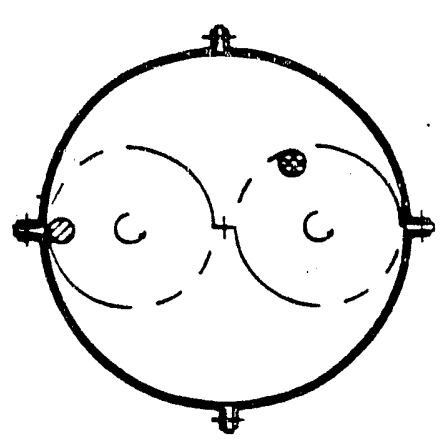

CIRCULAR CAVTTY

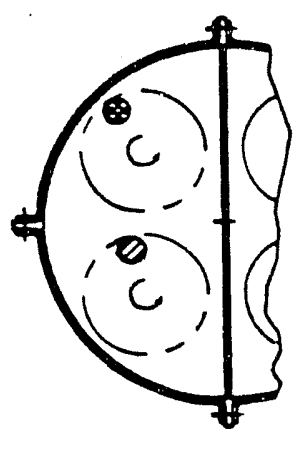

SEMI-CIRCULAR CAVTIES

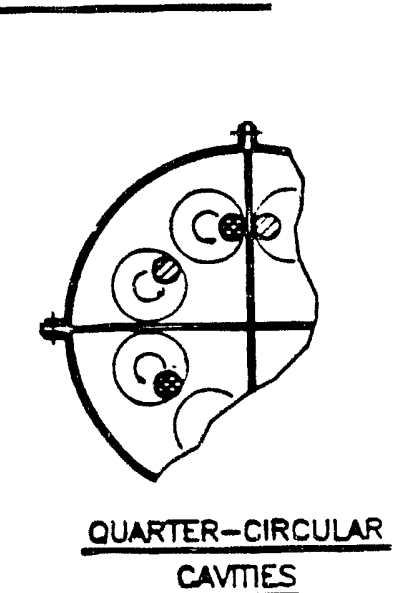

CAVTIES

Fig. 14. Approximate location and size of the two-rod motions for circular, semi-circular and quarter-circular cavities

We propose to test this tuning scheme in the cavities described in the previous section. 

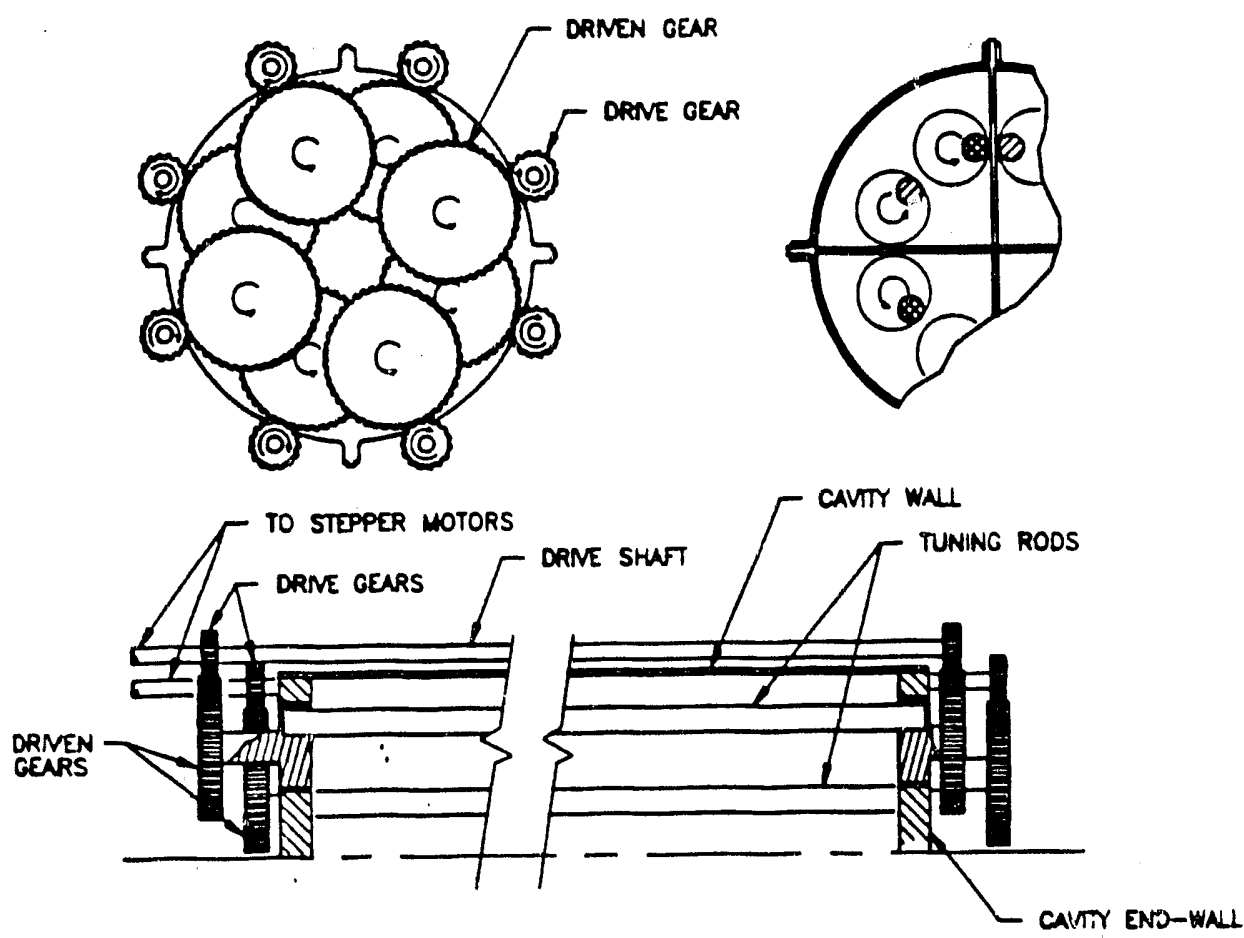

Fig. 15. Schematic design for transmission of the tuning mechanism.

\section{Cavity synchronization}

The multi-cavity arrays will work only if all are at the same frequency (within a tenth of $1 / Q$ ). We plan to use one of the cavities as the "master" and the remainder as "slaves." The resonance of the master will be set and its $Q$ determined just as is now done with a single cavity. Then each of the slave cavities will be adjusted to maximize its transmission at the resonant frequency of the master cavity. Peaking up ihe signal in this way should be a relatively rapid procedure. Once the cavities are synchronized they will stay together for a number of spectra, so long as the tuning rod mechanisms are sufficiently precise. Thus the multi-cell cavities can probably be tuned by dead-reckoning for 10-20 spectra before resynchronization becomes necessary.

During the next year it is proposed to build and test such a multiple cavity tuning apparatus. The cavities would be the cylinder partitioned into two and into four sections described above. The cavities would have individual stepping motors and gears and would be synchronized under computer control. 


\section{E. Combining cavity outputs}

In the multi-cavity setup, the outputs of all the cavities need to be brought in phase to the input of the microwave amplifier. As discussed above, this means that all the cavities must have the same resonant frequency, the same coupling $Q$ (i.e., $Q_{h}$ ), and identical lengths of RF cable between each cavity and the power combiner.

We propose to demonstrate this capability using the test cavity setup descrihed above, combining first 2 and then 4 cavity outputs. A test signal will be injected into the cavities to simulate axion $\rightarrow$ photon conversion and the effciciency of detection of this signal measured.

\section{F. Cryogenic amplifier}

We plan to use cryogenic microwave amplifiers manufactured by Berkshire Technologies, ${ }^{11}$ or other sources. Between 1 and $2 \mathrm{GHz}$, noise temperatures around 3-4 $\mathrm{K}$ are available. ${ }^{6}$ (See Fig. 16 and Fig. 17.) According to Berkshire, HEMT amplifiers with excess noise temperatures $\sim 5 \mathrm{~K}$ can be made over the $200 \mathrm{MHz}-1 \mathrm{GHz}$ range as well. During the next year, we plan to acquire one such amplifier and test its noise properties. 


\section{REFERENCES}

1. The Decade of Discovery in Astronomy and Astrophysics, National Research Council (National Academy Press, Washington, 1991).

2. P. Sikivie, Phys. Rev. Lett. 51, 1415 (1983).

3. P. Sikivie, Phys. Rev. D 32, 2988 (1985); L. Krauss, J. Moody, F. Wilczek and D. Morris, Phys. Rev. Lett. 55, 1797 (1985).

4. S. DePanfilis, A.C. Melissinos, B.E. Moskowitz, J.T. Rogers Y.K. Semertzidis, W.U. Wuensch, H.J. Halama, A.G. Prodell, W.B. Fowler and F.A. Nesrick, Phys. Rev. Lett. 59, 839 (1987).

5. C. Hagmann, P. Sikivie, N.S. Sullivan and D.B. Tanner, Rev. Sci. Instrum. 61, 1076 (1990).

6. C. Hagmann, P. Sikivie, N.S. Sullivan and D.B. Tanner, Phys. Rev. D 42, 1297 (1990).

7. D. Morris, private communication.

8. W.U. Wuensch, S. DePanfilis-Wuensch, Y.K. Semertzidis, J.T. Rogers, A.C. Melissinos, H.J. Halama, B.E. Moskowitz, A.G. Prodell, W.B. Fowler, and F.A. Nezrick, Phys. Rev. D 40, 3153 (1989).

9. A.C. Melissinos, B.E. Moskowitz, J.T. Rogers, Y.K. Semertzidis, S. DePanfilisWuensch, W.U. Wuensch, H.J. Halama, A.G. Prodell, W.B. Fowler, and F.A. Nezrick, in Cosmic Axions edited by C. Jones and A. Melissinos (World Scientific, Singapore, 1990), p. 39.

10. C. Hagmann, P. Sikivie, N.S. Sullivan and D.B. Tanner, in Cosmic Axions edited by C. Jones and A. Melissinos (World Scientific, Singapore, 1990), p. 42.

11. Berkshire Technologies Inc., Oakland, CA 94609.

12. Passive Microwave Technology, Camarillo, CA. The models used were LTA1219KS (1.3-1.7 GHz) and LTA1267KS (1.7-2.2 GHz).

13. Chimera system with AD16 option, Atlanta Signal Processors Inc., Atlanta, GA 30308.

14. Texas Instruments TMS320C25 digital signal processor.

15. Our limit is not valid in the narrow window $1.346096-1.346382 \mathrm{GHz}$, where a mode crossing occurred and where no data could be obtained.

16. Triangle Microwave, E. Hanover, NJ. 


\section{PERSONNEL}

The co-principal investigators on this proposal are Pierre Sikivie, Neil Sullivan, and David Tanner. This year, Paul Avery and john Yelton are considering joining the fullscale experiment and are attending our group meetings. Pierre Sikivie and David Tanner will contribute approximately $16 \%$ of effort to the project. Neil Sullivan, whose duties as Department Chair reduce his time for research will contribute $2 \%$ of his summer effort and $3 \%$ during the academic year. We propose that the project director for the UF pilot project be David Tanner.

We intend to involve a graduate student $100 \%$ time on the project. The student currently working on the project is Chad Davies, who passed the Departmental Qualifying Exam this fall.

We also anticipate extended visits by two collaborators from the INR. Details of the plans by this group for participating in the cavity development are given in Appendix A. We will need to provide support for these visitors during the time they are in Gainesville. 
Appendix. Letter from INR Collaborators

The following letter from Vadom Kuzmin outlines the plans of the Institute for Nuclear Research of the USSR to contribute to the cavity development:

Date: Fri, 6 Sep 91 09:02:31 +0300 (EET)

From: kuzmin@inucres.msk.su (Vadim Kuzmin)

Subject: axion collaboration

To: pegasys@esa.slac.stanford.edu

Cc: sikivie@ufpine.bitnet

Message-id: <AAdlmneqdE@inucres.msk.su>

Organization: Institute for Nuclear Research of USSR Acad.Sci., Theoretical Physics Division

September 6, 1991

Dear Professor K. van Bibber,

Thank you for your message of August 30, 1991. On your request we are sending you the following information concerning the participation of the Institute for Nuclear Research of the Academy of Sciences of the USSR in the axion search experiment.

1. The following team is to participate in the experiment:

Golubev Nikolai

Kuzmin Vadirn

Pashenkov Anatoly

Kazachenko Oleg

Polushkin Valery

Bleule Alexander

Geraskin Eugeny .

Of these, N. Golubev, O. Kazachenko, A. Bleule and E. Geraskin are deeply involved in the neutrino mass experiment at INR (integral electrostatic spectrometer with magnetic adiabatic collimation based on superconducting magnets), V. Kuzmin ia a theorist, A. Pashenkov is involved in the Kaon factory project at INR and is responsible for design and testing of RF cavities (to be made of ceramics and others) for the Kaon factory accelerator and V. Polushkin is an expert in lasers, crystal growth and fabrication of high precision optical and mechanical systems.

It should be understood that this team may be subject to changes in due course, in particular, new participants may have to be included.

2. We anticipate the following preliminary schedule for the first stage of the preparation of our contribution to the experiment :

a) Fabrication of the prototype cylindric cavities made of $\mathrm{Al}_{2} \mathrm{O}_{3}$ ("Kodor") ceramics (vacuum suitable) with internal surface covered by oxygen-free high purity copper. The sizes are : internal diameter $152 \mathrm{~mm}$, the wall thickness $4 \mathrm{~mm}$, length $340 \mathrm{~mm}$. We are going to make at least two such prototypes by October 10,1991. These have been already ordered in industry and paid by INR. The precision of fabrication of the internal surface is guaranteed to be Delta $R=5.10^{-4} \mathrm{~m}$ and the quality of the internal ceramic surface before covering by copper is to be 0.1 micrometer. 
b) Testing of one of these prototypes at temperature 4.5 K in Moscow by November 10, 1991.

c) Testing another prototype at the pilot experiment installation of the UF collaboration or at any other analogues set up in the U.S. (if necessary, at temperature $1.7 \mathrm{~K}$ ). This possibility and the schedule is subject to discussions with the American participants of the collaboration. On our side we suggest this testing to be completed as soon as possible (by the end of November ?) We think it is important that representative(s) of the INR team participate in this testing.

d) Fabrication of the full scale propotype of these cavities that are to be used for study of the high frequency part of the axion search interval. The sizes are : internal diameter $375 \mathrm{~mm}$, wall thickness $5 \mathrm{~mm}$, length $1100 \mathrm{~mm}$. The same material and precision as in item (a). Tr, be completed by the end of November, 1991 .

e) Testing of the full scale prototype at temperature $1.7 \mathrm{~K}$ is yet unresolved problem. We are studying the possibility to test this prototype at $4.5 \mathrm{~K}$, but going down to $1.7 \mathrm{~K}$ is very hard (if not impossible) at INR. This is one of the important points to be discussed when you are at INR. Also, we should discuss the compatibility of testing procedures that are going to be used by the American and Soviet parts of the collaboration (this applies also to items (b) and (c).) We hope this testing could be completed by the middle of February, 1992.

f) Fabrication of the full scale prototypes of $1100 \mathrm{~mm}$ cavity divided onto 2 and 4 sections. Schedule : by the end of June, 1992 (subject $t:$ results of testing, item (e).) Testing of these prototypes see item (e).

g) We are ready to discuss the participation of the INR team in the design and fabrication of the tuning mechanism of cavities (rods, mechanics, etc). The responsibilities and the time schedule is another major point to be discussed during your visit to INR.

h) Fabrication of the installation consisting of 8 cavities $1100 \mathrm{~mm}$ (undivided into sectors) by the end of June, 1992. In summer 1992 these could be shipped to LLNL for assembling.

i) Fabrication of 16-fold and 32-fold installations by the end of September, 1992. Alternatively, there may be a possibility to use unified cavities that may be divided both into 2 sectors (16-fold set up) and 4 sectors (32-fold set up). This should be clear by the end of June, 1992.

In parallel, we are going to design the installation with the length $2200 \mathrm{~mm}$ consisting of two 1100 $\mathrm{mm}$ sections "glued" together. We would like to discuss with you during your visit to INR effects of irregularities of the cavity in the place of "glueing" as well as effects of combining two cylinders into one that may cause the lost of precision. We hope the design of long cavities could be finished by the end of June, 1992.

We think that the above items should constitute the first stage of the preparation of our contribution to the experiment. If you agree with the general features of this schedule, we are ready to start the procedure for getting the project financed by the USSR bodies.

We together should understand that the standard way of collaborating between INR and U.S. laboratories is such that the living expences and travel within the U.S. and U.S.S.R. of Soviet and American scientists, respectively, should be covered by the receiving institutions. The same applies to the transportation of the equipment. 
Other matters of mutual interestshould be discussed during the preparation of the agreement of collaboration that should be started soon.

Yours sincerely,

V.A. Rubakov

Vice-Director of INR,

N.A. Golubev, V.A. Kuzmin

for the INR team 


\title{
Computer Acquisition for
}

\section{Research in Theoretical and Experimental High Energy Physics}

\section{Task S}

Principal Investigators:

\author{
Paul Avery and J. Yelton \\ Physics Department \\ University of Florida \\ Gainesville, Florida 32611
}




\begin{abstract}
We present a proposal to DOE to improve our existing computer system. We are requesting funding to (1) augment the disk capacity of the system; (2) pay $50 \%$ of the salary of a system manager/programmer and (3) cover $50 \%$ of maintenance costs. A significant number of matching funds have been obtained from the University of Florida.

\section{Recent history}

We presented a full history of our computer acquisition at UF in our 1991-92 Task S proposal to DOE. As discussed in that report, we traded back our VAX 6220 and 11 Gbytes of disk to DEC in exchange for a large Unix system consisting of 17 DECstations 5000/200s, of which 12 were connected by a FDDI fiber optic ring and acted as a processing farm. The trade relieved us of a system that was expensive to maintain and upgrade, and gave us approximately 300 Vax equivalents of computing power ( 215 in the servers alone). DEC funded the trade by extending their External Research Agreement with us in exchange for our development of the UFMulti parallel processing system. We purchased two Vax 3100s with 2 Gbytes of disk in order to replace the 6220 server and acquired 8 Gbytes of SCSI disk for our Vax 8x3200 farm and 6 Gbytes for the Unix system. The disk came with a 5 year warranty and thus did not need to be maintained.

Between the time that proposal was written and now we have made a number of changes to the system. Our philosophy during this time has been to trade in VAX/VMS computers for Unix machines and reduce maintenance costs wherever possible. Amazingly enough, VAX computers have been in such demand that we have had no trouble exchanging them in at little extra cost for Unix machines having far more computing power and twice the memory. The changes are summarized below.
\end{abstract}

1. A reduction in price permitted our trade of the VAX 6220 and disk drives to net us 7 more machines for a total of 24 DECstation 5000 s.

2. We traded our $8 \times 3200$ Vax farm ( 25 Vax equivalents) for 4 DECstation 5000 s ( 72 Vax equivalents), almost triple the computing power of the Vaxes. The DECstations came with 16 $\mathrm{MB}$ of memory and FDDI connections.

3. The disk on the Vax 3200 farm was transferred to the Unix system ( 7 Gbytes) and to the VAX servers (1 Gbyte). Because CLEO was taking data at a very high rate, we purchased an additional 10 Wren 8 drives ( 13.5 Gbytes) and 5 Wren 7 drives ( 5 Gbytes) to add to our Unix system. All disks come with 5 year warranties ( $\$ 90$ extra) and are not maintained.

4. We exchanged 4 VAX 3200 s ( $8 \mathrm{MB})$ for $4 \mathrm{VAX} 3100 \mathrm{~s}^{\prime}(16 \mathrm{MB})$ at no cost. This trade was made to avoid the much higher maintenance costs of the 3200 s.

5. Since we have so many identical machines in the Unix farm, we were able to slash maintenance costs by getting a Return to Manufacturer contract for all the DEC 5000s to 
replace our onsite contract. We are still maintaining the Vaxes onsite because they are used as desktop machines or servers.

6. We acquired 2 NeXT computers to aid us in our collaboration with the SLAC-led GISMO group in developing a new Monte Carlo package that will eventually replace GEANT. This work is described later in the proposal.

7. The Department of Physics provided us with a computer room in our building which we share with High Energy Theory, Condensed Matter Theory and Astrophysics. It has enough power and air conditioning for significant upgrades.

\section{Present configuration of computer system}

The system has the following components (see Figure 1):

1. Unix processing farm: 23 DEC 5000's (16 MB), of which 19 are linked by FDDI fiber optic cable. Theoretically, FDDI provides a useable $1 / O$ throughput of roughly 20 times times that of Ethernet and can be considered to be a fast $1 / O$ bus. The location of disk resources becomes less of a crucial issue when the processors are linked by fiber and provides a way for us to carry out our DST multiprocessing jobs with far better I/O capability.

2. 5 desktop DEC 5000's (16 MB) with 16 inch color screens. These are also part of the Unix farm, but are not used for this purpose as heavily as the dedicated machines. We also have a DEC 3100 that we use for development work. We will be bringing this system up to Cornell to use in the Florida office.

3. 32 Gbytes of disk attached to the DEC 5000s. 29 Gbytes of this is used for scratch storage for CLEO Il data and Monte Carlo simulations. The disk is distributed around the computing farm so that multiprocessing jobs using UFMulti can effectively parallelize their I/O. This is very important for CLEO physics analysis which involves several Gbytes of data being processed on each pass.

4. 2 VAX 3100 model $38 \mathrm{~s}(24 \mathrm{MB})$ which are used as servers for the VAX system. These have approximately 6 Gbytes of disk attached.

5. 1 VAX $3200(8 \mathrm{MB})$ used for our HEPnet connection to Fermilab.

6. 7 VAX 3100 workstations 3100 s. Most of these are used as desktop machines in the experimental and theoretical groups.

7. 2 NeXT computers (20 MB) used for our collaboration with SLAC on the GISMO project. Each of these have $400 \mathrm{MB}$ disks.

8. $28 \mathrm{~mm}$ Exabyte drives, $24 \mathrm{~mm}$ DAT drives and $2 \mathrm{CD}$ readers. These are split between the VAX and "Inix systems. 
We have found the DEC 5000 to be a well balanced, cost effective machine, especially with the FDDI fiber connections. Our benchmarks show that we can read locally attached SCSI disk drives (WREN VII) at a real rate in excess of $1 \mathrm{MB} / \mathrm{sec}$, using Fortran reads. Unfortunately, we have achieved only about 1-2 Mbytes/sec transfer rate on the FDDI, a fraction of its theoretical bandwidth. We are exploring the situation with DEC and hope to be able to tune the system parameters in a way that will allow us to take better advantage of the system. This is important for our CLEO analysis because many multiprocessing jobs are I/O limited.

We have two connections with the outside world: (1) a dedicated 9600 baud line to Florida State University which routes the connection directly to Fermilab and HEPnet and (2) a connection via several $\mathrm{T} 1$ lines to NSFnet. The second connection is free to us since it is maintained by the University.

The above list demonstrates quite vividly that we have moved from a VAX/VMS computing environment to one dominated by Unix. This has happened during a year in which CLEO switched to Unix for almost all of its computing. The total useable computing capacity of our Unix farm is rouglily 500 Vax equivalents (415 dedicated and 90 on desktops), when the I/O rate is not saturated.

\section{How the system is being used}

A large amount of analysis has been carried out on both the Vax farm (before it was traded) and on the Unix machines as described later in this section. The experimental HEP group has taken the leadership role in departmental computing, with one us (Avery) the chair of the Computing Committee. We have provided accounts for many members of the department as a good will gesture to allow then to carry out low level activities such as word processing and e-mail. This in turn has made it easier for us to press for maintenance funds.

Our analysis activities for the past year are detailed below.

1. The $8 \times$ Vax 3200 farm was used heavily to generate GEANT Monte Carlo samples for D0 (this work has now been superseded by the SGI machines acquired by Fermilab). We have stopped doing this work since Andy White's departure in September 1991.

2. We carried out approximately $3 / 4$ of the entire CLEO Pass 2 data reduction on our Unix farm, just in time for the 1991 summer conferences. This work took approximately 2 months on half the farm.

3. We carried out extensive simulations for the CLEO B Factory, most of them on the VAX farm. 
4. We are now generating massive event samples for the CLEO Monte Carlo program. This work is being carried out at a number of CLEO member institutions, but we have the largest computing capacity to devote to it. This will take approximately 3 months.

5. We have been using the Unix farm for physics analysis for quite some time. All our analysis programs have been converted over to Unix and we greatly benefit from the fact that the entire CLEO II data sample is on disk and can be accessed remotely without tape mounting. We have given accounts to CLEO members pursuing tau, charm and B meson analyses because the Cornell VAX and SGI 3/380 are almost completely saturated. They are pursuing getting more CPU resources but in the meantime we are providing our colleagues with a way to get their analyses done.

6. Rick Field conducted research related to the SSC and the SDC. He carried out a large number of Monte Carlo simulations for SDC. As a member of the "forward calorimeter" subgroup of SDC, he examined several generic questions conceming the nature of jets in $p-p$ collisions at the SSC. Some of the work was done in collaboration with Mike Barnett (head of the subgroup) and Andy Whise and resulted in an SDC note entitled "Jets in the Forward Region".

\section{Our plans for this year}

\section{Computing}

1. We will be redoing the entire CLEO Pass 2 data reduction during the first quarter of 1992. The ongoing Pass 2 work will be carried out at Cornell on their SGI 3/380. We expect the recompress to take approximately 3-4 months.

2. Our CLEO Monte Cario work will continue, since a large number of $B$ events (comparable to the data sample) need to be simulated for the background studies.

3. We will be using the Unix farm for our B analyses, especially those that require large combinatorics, such as $B->$ Dn $\pi, D^{*} n \pi$. Our charmed baryon analyses will also be carried out on the system. These projects will use our UFMulti parallel processing software to best take advantage of the total processing power on the system.

4. Other CLEO members will also be pursuing analyses on the Unix farm. We will be collaborating on some of these analyses, primarily the ones involving B decays. These projects will also use UFMulti.

5. We are collaborating with University of Mississippi to carry out SDC simulations jointly on our respective farm (they recently acquired a large DECstation 5000 farm for their E-791 data reduction). The work has not started yet, but we hope to begin in late Fall, 1991 after the iogistics are workeu uet. Tius wüh is úiñg dunc inder the auspices of SALIEP, Southern Association for High Energy Physics. The HETC package is used here to simulate the calorimeter time response, something that is impossible to do with GEANT at present. As part 
of our work, we will incorporate UFMulti in their simulation in order to take advantage of multiprocessing.

6. Ric : Field will be extending his SSC related research and Monte Carlo simulations. At present, he is looking into the imporiance of of the forward region (eta > 3) in determining the missing transverse energy. Missing transverse energy is an important signal for new physics and requiring large missing transverse energy is a well known way to cut away the expected QCD background. He will be performing SDC detector simulations in addition to his standard physics generation.

Obviously, our ability to carry out all these tasks depends to a great extend on the organization of the system, and will be circumscribed by the total computing capacity and disk size. We will be augmenting these during the coming year.

\section{UFMulti development with SSC}

The UFMulti software system we developed at UF has been the cornerstone of our computing effort since 1988 . With UFMulti a single user can use the entire computing capability on a network, even when it is in the form of individual workstations. This has allowed us to take advantage of the microprocessor revolution to acquire a large amount of computing power at low cost, because we do not need the expensive high bandwidth buses used in almosi all parallel processor designs. Our development of UFMulti also helped generate funding for computer resources and enabled us to negotiate better discounts from computer vendors.

As promised in our previous proposal, we have ported UFMulti to Unix and can now routinely run HEP jobs using 15-25 processors for rapid turnaround time. This turned out to be no small achievement because Unix is quite different than VMS in many subtle ways. The work was carried out by Greg Huey and Chandra Chegireddy.

Our experience after three years is that UFMulti is an invaluable tool for doing physics analysis but it is limited in some important ways. For example, we wrote the interprocess communications interface ourselves using DECnet protocols with the intention of providing a TCP/IP driver later. We took this route because no standard communication software was available at the time (1988-89). However, after receipt of our SSC Subbsystem Award we agreed to rewrite UFMulti so that it would be based on the Cooperative Process Software (CPS) developed at Fermilab. This step in turn would better integrate UFMulti into in the SSC environment which uses CPS for all parallel processing. Additionally, it would drastically simplify UFMulti since we would not have to worry about the communications interface and allow us concentrate on computing issues.

After much thought, we have decided to completely rewrite UFMulti. This decision is based on what we have learned in the past three years about computing, particularly distributed processing and object oriented programming. Accordingly we will write UFMulti in $\mathrm{C}++$ (it is currently written in Fortran and C) but it will remain callable from $\mathrm{C}$ or Fortran. Using $\mathrm{C}++$ 
will make UFMulti extensible (i.e., users can easily add features to it) and enable us to better integrate it with GISMO and CABS, both of which are written in $\mathrm{C}++$.

As a first step in the rewrite we will base UFMulti on the Fermilab CPS system and gain experience running large HEP codes on the PDSF at the SSC with the new package. This initial effort wil be a collaborative effort with the SSC Computing Division since we intend to modify CPS to add new capabilities, e.g., priority messages, hooks for monitoring distributed jobs from outside. The SSC people have agreed that either they or the Fermilab Computing Division will integrate our changes into CPS. This project is already underway and will finish before the proposal period starts.

Integrating our software with CPS wili make UFMulti much more flexible in that it can handle a much larger set of problems. This in turn means that we need to add more intelligence to the software. For example, we have almost succeeded in providing a mechanism in which UFMulti decides in a multiprocessing job which CPUs should read from which disks, taking advantage of knowledge of whether a disk is connected directly, by FDDI or by Ethemet to a given processor. This allows UFMulti to optimize the I/O transfer rate for the job as a whole.

We will also add monitoring capability to UFMulti, in the sense that a user can merely query the system about the state of a particular job and get back job statistics, histograms, etc. This is extremely important in interactive or semi-interactive analyses.

We are investigating the possibility of replacing CPS's communication software with vendor supported tools such a.s NCS (Network Computer System) which is being adopted as a standard by most of the major computer companies (e.g., DEC, IBM, Silicon Graphics, HPApollo, etc.) for a distributed computing environment. NCS is very inexpensive and lias a very nice set of tools that make it unnecessary for physicists to design communications protocols. SSC has already indicated its qualified support, since they are not committed to CPS in the long term.

\section{The GISMO project}

We have just begun collaborating with physicists from SLAC and CERN on a project to develop a new Monte Carlo Simulation package. The project is called GISMO and is led by Bill Atwood of SLAC. The intention is to develop a simulation package based on object oriented principles. About 20 or so physicists from SLAC, CERN and several scattered institutions are involved. Working code was originally developed on NeXT machines written in Objective-C, an extension to $C$ that permits object oriented programming (OOP) constructs.

The major goals of the project are: (1) Base it on a geometry used in CAD systems for better flexibility; (2) allow graphics (color) to be developed with the code for better diagnosis; (3) develop user changeable graphical user interiaces (menus) so that simulations can be run or at least started interactively; (4) use OOP principles (it is being written in $\mathrm{C}++$ ) in the code design to simplify the process and provide extensibility; (5) use existing "black box" code such as EGS4 whenever possible to save deveiopment time and avoid writing everytining from 
scratch; (6) make it efficient so that it cannot be mistaken for GEANT; (7) make it machine independent within the Unix operating system.

The long term goal of GISMO is to eventually replace GEANT, although this will certainly not happen in time to affect the next round of SSC proposals.

We are involved in the following subprojects: (1) port the graphical user interface from NeXT to Motif in a general way; (2) help develop a general track class that will be used everywhere, from the event generator (MC++ from LUND) to the tracks used in phyusics analysis; (3) provide distributed processing capability so that GISMO can automatically take advantage of parallel processing. We expect that these tasks will not take enormous amounts of time. Chandra Chegireddy is handling the menu interface and Paul Avery is working on the track class with Toby Burnett of $U$ of Washington. Chegireddy and Avery will both work on the distributed part of the problem when the package can be run on a general Unix machine.

\section{The CABS project}

We are working with Nobu Katayama and Dan Riley of Cornell in extending the physics analysis package CABS (CLEO Analysis by Script) written by Katayama. Our intention is to fully integrate kinematic fitting (written by Avery) and multiprocesing to make CABS a complete analysis system similar in spirit to PAW but much more advanced. As it is currently written, a user provides a file written in "Script" describing the analysis to be carried out directly in terms of physics concepts. A program (compiler) translates this information into $\mathrm{C}++$ which is then compiled on the host machine and executed.

Being a preprocessing system, CABS suffers from the lack of debugging tools that plague all such systems. We are proposing to provide direct programming support for the physics concepts but in such a way that the user can use $\mathrm{C}++$ directly rather than through the Script file. This provides a leaming path: use Script until you are familiar with $\mathrm{C}++$ and then use $\mathrm{C}++$ directly. 


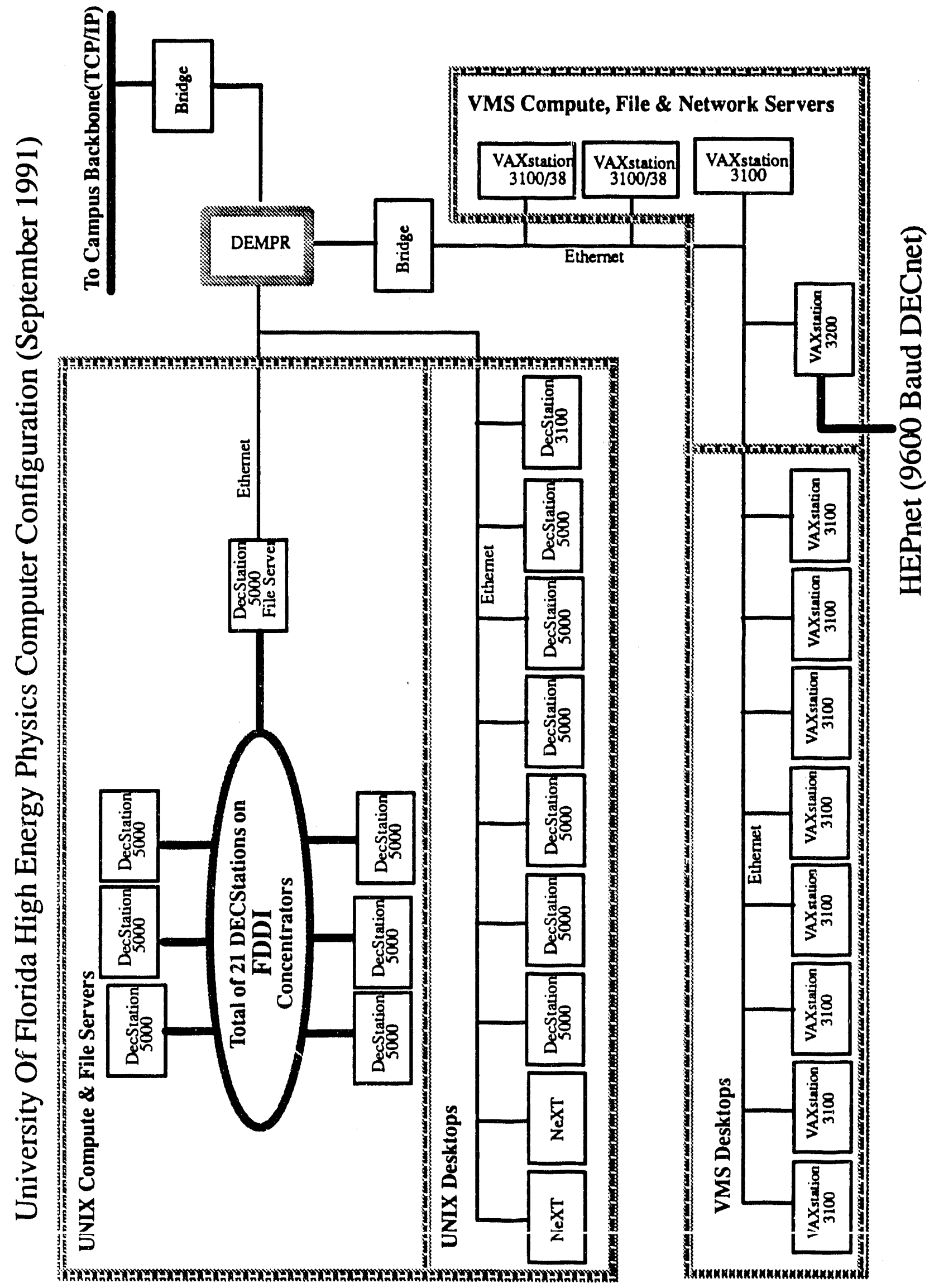




\section{TABLE OF CONTENTS}

I. Introduction

II. Accomplishments to Date

III. Work Plan for 1991-1992

Task I Producing Polymers and Scintillator

Task II Life Testing Materials Produced by U. of F. 47

Task III Life Testing Materials Produced by SSC Community 55

Task IV Study of Injection Molding of Scintillator 57

IV. Facilities Available 73

V. Personnel 74

$\begin{array}{ll}\text { VI. Budget } & 78\end{array}$

$\begin{array}{ll}\text { VII. Budget Justification } & 79\end{array}$ 


\section{Introduction}

Five years ago the Detector Development Group at the University of Florida initiated a study which was hoped would lead to plastic scintillator unaffected by high (10 MR) radiation doses. Up to last year, it appeared that we could achieve that objective with the restriction of having to use an elastomeric plastic matrix. Dow Corning attempted to commercialize the technology.

Since "rubbery" plates may be undesirable in some circumstances, we have concentrated, during the last year, on rigid thermoplastic materials. The basic objective was to apply the knowledge we had developed on the radiation damage mechanism, to make these materials retain transparency after an exposure of $10 \mathrm{MRads}$. In addition, the scintillator light output should be unaffected by that level of exposure. We have succeeded in producing heat processible rigid material with these desirable radiation resistant properties.

The key to achieving this success is using an optical polymer whose glass transition temperature, $T_{B}$, is

a) high enough above room temperature to ensure long term maintenance of mechanical properties yet

b) low enough to ensure a rapid relaxation of radiation induced stress.

We have found that a glass transition temperature in the range of $40^{\circ}-50^{\circ} \mathrm{C}$ appears optimal. We have termed such a material, a "low $T_{8}$ thermoplastic". A remarkable, and as yet not understood, result of lowering the $T_{B}$ in this manner, is that not only is there a rapid annealing of the radiation induced optical damage, but permanent damage is dramatically reduced. It is this latter result which is of great importance for plastic scintillator operation at the SSC. 
The general approach to making scintillator for the SSC is now apparent. Much work remains to be done on optimizing the material based on the important factors of
a) material properties
b) cost of materials
c) life testing
d) injection molding properties.

This proposal addresses these issues. In order to accomplish the last objective, we have hired a PhD chemical engineer who has specialized in injection molding of optical polymers and ordered an injection molding machine.

Continued funding of this project should result in the production of optical quality rigid plates of low $T_{\mathrm{g}}$ thermoplastic scintillator, whose properties are constant up to about 10 MRad exposure. 


\section{Accomplishments to Date}

The Detector Development Group at the University of Florida has identified the only known optically radiation hard polymer, scintillator and wavelength shifter materials. We summarize our findings here.

It has long been known that commercially available scintillator material undergo severe optical damage when exposed to high energy radiation. Five years ago the University of Florida initiated a program to identify optically radiation hard material for use in scintillator at the SSC. During year one, sol-gel glasses were studied as a scintillator base material. This work resulted in the discovery that the $\mathrm{Si}-\mathrm{O}$ bond was optically radiation hard

at exposures up to $10^{8}$ Rads. There were difficulties however in doping this material with fluors to produce stable scintillator and in producing large samples.

The next step in our program consisted of screening a large variety of optical polymers to characterize radiation damage to transmission. Polysiloxane with a Si-O backbone was found to be optically radiation hard. This is depicted in figure 1 where the transmission damage of polysiloxane is compared to that of PVT, PS and PMMA.

Over the next three years we conducted an extensive study of siloxane polymers using monomers of dimethyl, diphenyl and methylphenyl siloxanes. An important finding of that study was that polymethylphenylsiloxane is a candidate polymer for use at the SSC. However, the most important result of that work was the demonstration of the existence of optical polymers with extreme resistance to radiation damage. It held out the promise that other possibly more convenient polymers with similar properties could be identified.

Crystalline monomer centers present in siloxane polymers form submicron crystals that 
lead to Rayleigh scattering. We succeeded in purifying low viscosity liquid polymethylphenylsiloxane and demonstrated radiation hardness and optical clarity. These results are shown in figure 2 a,b,c. Figure 2a shows the transmission spectra of liquid siloxane before and after 10 Mrads. There is little transmission loss due to radiation. Figure $2 \mathrm{~b}$ and $2 \mathrm{c}$ compare the transmission of 1 and $5 \mathrm{~cm}$ samples of polymethylphenylsiloxane to that of polydimethyldiphenylsiloxane which is not as easily purified. Rayleigh scattering losses are evidenced by a wavelength dependence of $\lambda^{-4}(1)$.

During this past $11 / 2$ years we have been collaborating with Dow Corning in this effort. In the past six months Dow Corning synthesized a series of methylphenyl siloxanes. The object was to increase the molecular weight as compared to the liquid siloxane, and to add functional vinyl groups to the monomer. These vinyl groups cross-link with methyl groups to yield an elastomeric material that is suitable for injection molded plates. Dow Coming is currently producing plates from this material. Figure 3 shows the transmission spectra of such a plate before and after radiation. This material shows superior radiation resistance.

Another reason for selecting polymethylphenylsiloxanes is fluor solubility. In the past, we had had difficulty in achieving adequate solubility of fluors in siloxane polymers. Solubility is characterized by the solubility parameter $\delta$. The solubility parameter is defined (2):

$$
\delta\left(\mathrm{J}^{1 / 2} / \mathrm{cm}^{3 / 2}\right)=\left(\mathrm{E}_{\mathrm{coh}} / \mathrm{v}\right)^{1 / 2}
$$

where $\mathrm{E}$ is the coliesive energy and $\mathrm{V}$ is the molar volume. These parameters are readily calculated by group contribution methods. The closer the solubility parameter of fluor to 
polymer, the higher the fluor solubility The solubility parameter of

polymethylphenylsiloxane is 18.4 . This matches the solubility parameter of polystyrene, 18.6.

Previous siloxanes had solubility parameters between 15.5 and 16.7. Fluors were not

adequately soluble in siloxanes with low solubility parameters. We successfully dissolved $1 \%$ by weight of oligophenylene primary dye and $0.1 \%$ TPB secondary dye in

polymethyephenylsiloxane. However, due to the high mobility of dye in the flexible siloxane matrix and the high dye concentrations, the fluors migrate to the surface of the plate and crystallize. Dow Corning is currently developing the ability to covalently bind the dyes to the polymer matrix.

To summarize, over the past year the first high viscosity, transparent, radiation hard siloxane with high fluor solubility was processed into prototype plates.

In the past $11 / 2$ years, we also conducted research defining the mechanism of radiation induced discoloration of optical materials. It has been known for many years that when many glassy optical polymers are exposed to ionizing radiation, they discolor. In the presence of oxygen, this discoloration anneals by a moving boundary phenomenon. That is, a sharp border moves from the edges of the polymer inward, leaving colorless, annealed material behind. Many observers had interpreted the moving boundary to result from the diffusion of oxygen which reacts with the annealable color center to produce a colorless moiety. We showed that there is a physical plus chemical origin to this moving boundary. This important discovery was presented at the SSC Fort Worth meeting in October 1990.

We determined that when radiation discolored samples are heated above the glass transition temperature, a rapid recovery ensues. No sharp boundary is noted. Diffusion of 
oxygen is not instantaneous, even above $\mathrm{T}_{\mathrm{g}}$. Rather, the recovery is due to relaxation in the polymer matrix. In a more rigorous analysis, the kinetics of boundary motion were analyzed. The border movement was monitored in PMMA and in PS at different temperatures. The results are shown in figures $4 a$ and $b$. The boundary motion is thermally activated yielding an activation energy of $10 \mathrm{Kcal} /$ mole. All of this yields information as to the michanism of recovery. First, consider that such sharp boundaries are not observed in examples of pure Fickian diffusion. In addition, the boundary moves linearly with time. Linear boundary movement in polymers has been shown to occur when relaxation processes control kinetics $(3,4,5)$. For example, when organic liquids swell polymers, the matrix must relax to accommodate the penetrant. Here, the relaxation controlled boundary movement is linear with time. This fact, along with the fact that above $T_{\mathfrak{g}}$, where relaxations are rapid, annealing is also rapid, led us to believe that relaxations control recovery kinetics. Clough (6) pointed out that polymers recover above $T_{b}$ whether or not oxygen is present. He pointed out that there may be two mechanisms for recovery, one that operates with oxygen and one without. This hypothesis is in agreement with our initial experiments. We put forth two simple models:

$$
\begin{aligned}
& \mathrm{O}_{2}+\mathrm{A} \rightarrow \mathrm{O}_{2} \mathrm{~A} \\
& \mathrm{~A}+\mathrm{A} \rightarrow \mathrm{A}-\mathrm{A}
\end{aligned}
$$

where A denotes a color center and A-A is colorless.

One immediate way of rendering polymers radiation hard became apparent from the above experimentation. Any polymer with $\mathrm{T}_{B}<\mathrm{T}$, where $\mathrm{T}$ is the normal operating test temperature, should not form anneable color centers. We started with two model polymers, 
PMMA and PS. These polymers have glass transition temperatures at about $100^{\circ} \mathrm{C}$, By appending large side chains on the polymers, the glass transition temperature drops below room temperature. These structures are shown in figure 5. These polymers, when irradiated to $10 \mathrm{MRad}$ over a period of three days and measured immediately after irradiation show exceptional radiation hardness. The results are shown in figure 6 .

Note: In siloxane, PS, PMMA, PVT, etc., part of the radiation damage is not recoverable. A bathochronic shift at short wavelengths induced by radiation is irreversible. Damage which cannot be annealed is said to be due to type II color centers. Annealable color centers are termed type I centers. Figure 7 shows the transmission spectra of polystyrene before, during and after recovery. Non-annealable regions in the UV region are noted, even after 30 days recovery. The low $\mathrm{T}_{\mathrm{g}}$ styrene and methacrylate polymers, by contrast, show very little type II color center formation.

To summarize, we proposed a mechanism to account for radiation induced annealable color center formation in commercial scintillator polymers such as PS and PMMA. We produced analogues of these polymers with $T_{8} \leq$ room temperatures. These polymers are optically radiation hard. The University of Florida has applied for a patent on this breakthrough discovery (7).

We recognized that these low $T_{B}$ polymers can be used to manufacture fibers and plates, but special processing requirements must be taken into consideration. These polymers are viscous liquids and must be cross-linked to produce elastomeric polymers.

For plates, cross-linking is easily achieved during casting or by a reaction injection molding. For fibers, cross-linking can be achieved by adding cross-linker to the polymer 
before spinning. We have determined that stable fibers can be spun by encapsulating the viscous liquid with cross-linker and catalyst in a thermoplastic sheath. The fibers can then be post reacted with heat or UV light to induce crossing linking. All of this presents two problems: the first is that plates of these cross-linked elastomers are rubbery and therefore not self-supporting. The second is that curing of fibers after spinning requires an extra-timeconsuming step in production.

In summary, there is excellent radiation stability of these elastorneric polymers, with $I_{8} \leq$ room temperature but they are difficult to process into self-supporting structures. After the Forth Worth meeting we decided to concentrate on finding the conditions required to produce a radiation stable, heat processible rigid thermoplastic material. In the course of the last nine months we have succeeded. The $U$. of $F$. has applied for a patent on this discovery (8).

Recognizing that different thermoplastics recover at different rates, we focussed studies on the following:

1. the effect of total radiation dose on recovery rate

2. the effect of $T_{8}$ of the thermoplastic

3. the effect of polymer chemical structure

4. the effect of oxygen permeability

We summarize these findings as follows:

Figure 8 depicts the effect of total radiation dose on recovery velocities in polystyrene. The recovery after $3 \mathrm{Mrad}$ is over twice as fast as that after $20 \mathrm{Mrad}$. This prompted us to search for thermoplastic polymer structures that recover at more rapid rates than PMMA and 
PS. We focussed on reducing the glass transition temperature of the polymer while keeping the $T_{8}$ enough above room temperature to allow for retaining dimensional stability. We used a set of methacrylate polymers as model compounds. We varied the side chain on the polymer to induce variations in the glass transition. The following structures were investigated:

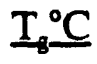

Poly (methylmethacrylate) PMMA

100

Poly (ethylmethacrylate) PEMA

66

Poly (isobutyl methacrylate) PiBMA

Poly (propyl methacrylate) PMMA

Poly (butyl methacrylate) PBMA

The boundary velocity at room temperature was measured after a dose of $10 \mathrm{Mrad}$ in 3 days time. PEMA recovered with a border velocity of $0.84 \times 10^{-5} \mathrm{~m} / \mathrm{hr}$ as compared to PMMA which recovered at a rate of $0.01 \times 10^{-5} \mathrm{~m} / \mathrm{hr}$. This factor of eighty-four increased recovery rate was astounding considering that the difference in $T_{8}$ was only $31^{\circ} \mathrm{C}$. It was therefore not surprising that all the rest of the samples showed no evidence of a radiation damage border after these dose conditions. Figure 9 shows the transmission spectra of PiBMA and PMMA before and after 10 Mrad. The damage is slight compared to PMMA. There is a small degree of recovery noted after 30 days in the case of PMMA. The following conclusions were drawn as a result of lowering the $T_{8}$ yet maintaining $T_{8}>$ room temperature of the thermoplastic:

1. There is a drastic enhancement of the recovery rate 
2. perhaps, most importantly, type II color centers are minimized as we had previously shown in the case of polymers where $T_{g}<$ room temperature.

There are important implications of these two conclusions for scintillating plastic for the SSC. Concerning the first conclusion, it may be said that plastics which rapidly recover from a short duration, but massive dose of radiation are not relevant to the SSC. This is because the SSC produces the massive dose over a very long period of time. Hence, rapidly recovering polymers hardly seem to be relevant. The real relevance and importance of our work lies in the second conclusion. Namely permanent damage to the pol mer is minimized. We have shown this to be an experimental fact at dose rates of the order of $10^{5}$ Rads/hour. We conjecture that: polymers with $\mathrm{T}_{8}=50^{\circ} \mathrm{C}$ will have minimal permanent optical damage even at the very low ( $\leq 10$ to $100 \mathrm{Rad} s / \mathrm{hr}$ ) dose rates encountered at the SSC.

The experimental verification of this conjecture is one of the important measurements which we propose to make in the coming year.

This experimentation constituted a major breakthrough in another way. It is now possible to use easily processible thermoplastics at the SSC. This invention encompasses the following methods of reducing the $T_{8}$ of ine polymer (8):

1. appending side groups which lower $T_{B}$

2. copolymerizing with a low $\mathrm{T}_{\mathrm{g}}$ polymer

3. blending low and high $T_{b}$ polymers

4. adding plasticizers to reduce $T_{8}$

We have, for example, added $20 \%$ dibiutyl phthalate plasticizer to PS samples which were irradiated to 10 Mrads. The $20 \%$ doped samples remaining transparent and 
consequently showed no recovery border. Whereas significant damage was noted in PS. The 1/2" diameter PS sample exhibited a sharp damage border which had not fully moved across the sample after 1 month. After the border had fully traversed the sample there remained substantial permanent optical damage at $<400 \mathrm{~nm}$ and reduced permanent damage at $>400$ nm.

We might note here that a major experiment at CERN used PMMA plastic with $15 \%$ naphthalene to make a scintillator. We have studied the effect of naphthalene and found that it reduces the $T_{8}$ of the polymer to about $50^{\circ} \mathrm{C}$. The CERN experiment operated for several years with a constant $( \pm 10 \%)$ light output. This important result indicated that plasticizer migration in and volatilization from a glassy polymer with $\mathrm{T}_{8} \approx 50^{\circ} \mathrm{C}$ is a negligible effect. This provides encouragement to give serious consideration to the simple and cost effective method of adding plasticizer to reduce $T_{B}$.

We also focussed on the effect of polymer structure on radiation damage. Consider that PS and PMMA which have similar $T_{8}$ 's $\left(\approx 100^{\circ} \mathrm{C}\right)$ show remarkably different recovery rates. PS has a room temperature boundary velocity of $1.1 \times 10^{-5} \mathrm{~m} / \mathrm{hr}$ versus PMMA with a velocity of $0.01 \mathrm{~m} / \mathrm{hr}$ after exposure to $10 \mathrm{Mrad}$. The polar PMMA matrix recovers more slowly. Thermoluminescence studies have shown that polarity has an effect on luminescence in irradiated polymers (9). We hypothesize that such an effect is due to the enhanced ability of polar polymers to trap electrons or radicals responsible for color centers in the matrix in voids.

Before further considering structure effects on radiation damage, we tum our attention to the effect of $\mathrm{O}_{2}$ permeability on radiation damage. Table I lists a series of polymers, $T_{z}$ 's 
recovery velocities and permeabilities. Permeabilities are roughly consistent with recovery values with the methacrylate series. PEMA is more permeable, however, than PS, but has a lower recovery velocity. PMMA has a relative permeability of 0.3 versus 2.3 for PS. The extremely low permeability of PMMA correlates with it having the lowest boundary velocity. However, permeability is influenced by the same factors that likely influence recovery ie. molecular packing and chemical structure of the polymer. We focused on a particular point; if PiBMA shows no recovery border is this phenomenon due to the fact that the $\mathrm{O}_{2}$ induced recovery reaction occurs more rapidly than we can measure it. Or, is a relaxation mechanism independent of $\mathrm{O}_{2}$ occurring? This point needed to be clarified. If $\mathrm{O}_{2}$ is necessary for recovery that occurs simultaneously with irradiation, any considerations of operating the plastic scintillator at the SSC in an inert gas environment need to be dismissed. With this in mind we polymerized two samples each of PS and PiBMA in vacuum. One sample of each polymer was removed from the vacuum vial. All four samples were irradiated to $10 \mathrm{Mrad}$. Both samples of PiBMA were clear while both samples of PS showed extensive color center formation. This proved that thermoplastics with reduced $T_{8}$ 's recover by a relaxation mechanism independent of $\mathrm{O}_{2}$. This means that it is possible to operate the SSC detector in air or an inert gas environment.

We continued to study the effect of structure on radiation sensitivity. We reasoned that if high $T_{8}$ polar polymers trap color centers in voids, lowering the surface free energy of the polymer should inhibit this trapping and result in more radiation hard optical properties. We synthesized the series of fluoralkylmethacrylates shown in table II. Surface free energies were calculated using the group contribution method developed by Quayle (10). We 
irradiated $1 \mathrm{~cm}$ thick samples of these polymers to $10 \mathrm{Mrad}$ in air. The area under the absorption curve before and after irradiation was plotted vs. surface free energy, $\gamma$, and vs. $T_{B}$. This is shown in fig. 10a and 10b. There is an obvious correlation between surface free energy and transmission. There is no direct correlation between $T_{B}$ and $\gamma$. No correlation between $\gamma$ or $T_{B}$ and transmission were noted before irradiation. This points to the fact that surface free energy and hence the structural chemistry also influences radiation hardness of polymers.

Figure 11 shows the transmission curves of polyheptafluorobutylmethacrylate, PhFBM before and after exposure to 10Mrad in an air environment. This sample has the lowest surface free energy of the polymers listed in table I, $36.9 \mathrm{dyn} / \mathrm{cm}$. It also shows the minimum radiation damage. These fluorocarbon polymers illustrate the effect of $\gamma$ on radiation damage. They are suitable for fiber cladding materials since fluorine substituents decrease the refractive index of the polymer. Poly(heptafluorobutyl methacrylate) has a refractive index of 1.38 . We propose the use of this material for cladding of read-out fibers especially in the end cap of the SDC where radiation levels are very high.

Polymer recommendations in the short term:

Our long term objective is the development of an optical polymer which 1) has low $\mathrm{Tg}$ (say $50^{\circ} \mathrm{C}$, 2) low surface free energy, and 3) has a high phenyl content. Based on the above data these criteria will yield the most stable radiation hard optical polymer suitable as a base for plastic scintillator.

As reviewed in the above, we have made considerable progress in the past year in identifying optically radiation hard thermoplastics. From this work, we recommend focussing on two polymers for scintillator, wavelength shifter and clear fibers:

1. Plasticized polystyrene for scintillating plates, clear fiber and wavelength shifting fibers and clear read-out fiber.

2. Radiation hard poly (isobutylmethacrylate) for clear fibers and wavelength shifting 
fibers and clear read-out fiber.

These are two simple systems that are radiation hard and heat processible in addition, we recommend $\mathrm{PhFBM}$ as a fiber cladding material. Studies proposed here will focus on sccelerated testing to predict long term stability and processing. First, however, we will review our recommendations for radiation hard fluors to be used in these systems.

\section{Fluor recommendations}

In the past we have conducted extensive studies on identifying radiation hard primary and secondary fluors. These studies are summarized in publications $(11,12)$. We present the main points here.

We have shown that oligophenylene chromophores as primary fluors are radiation stable. Organic groups appended to these fluors degrade upon irradiation and produce color centers. These groups, often alkyl groups, are appended to the molecule for two reasons:

1. to increase solubility in the menomer

2. to form bridged structures which exhibit a bathochromic shift and higher quantum efficiency

The optimum primary dye structure chosen on the basis of absorption spectra radiation hardness, quantum efficiency, fluorescent lifetime and solubility is:

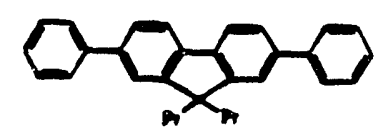

The emission maximum of this dye, OLIGO $374 \mathrm{~A}$ is at $374 \mathrm{~nm}$ which places it well above 
the region where permanent damage of the lowered $T_{z}$ polystyrene will cause any problem. We identified 1,1, 4-4-tetraphenylbutadiene, TPB, as a radiation hard secondary fluor:

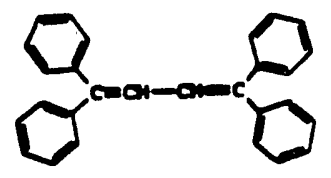

The absorption emission spectra of polystyrene, OLIGO 374A and TPB are shown in figures 12,13 and 14 respectively. The absorption spectra of $374 \mathrm{~A}$ overlaps the emission spectra of polystyrene.

The emission spectra of oligo 374, in turn, overlaps the absorption spectra of tehaphenylbutadine to produce high light output radiation stable scintillator. Figure (15) shows light output versus radiation dose for siloxane doped scintillator versus that of commercial scintillator. These dyes yield scintillator with light output that is stable up to 10 Mrad. These results were duplicated by our lab in two separate studies $(11,12,13)$.

TPB has an emission maximum at $450 \mathrm{~nm}$. The breadth of the emission spectrum extends from 430-500. We have identified two radiation hard dyes for use in wavelength shifting fibers. There are Coumarin 545 and Fluorol 76A. The absorption emission spectra of these fluors in thin films of PMMA are shown in figure $16 \mathrm{a}$ and $16 \mathrm{~b}$. The fluors are radiation stable and have absorption spectra which adequately overlap that of the secondary dye, tetraphenylbutadiene.

We conclude that fluor research is complete.

\section{Scintillator Light Output}

Light output of $2.5 \mathrm{~mm}$ thick low $T_{B}$ scintillator plate was measured using an 
Americium source and a Thorn EMI type 9907A phototube. The source was placed on top of the plate and the center of the P.M. cathode area. The light output was found to be constant to within $\pm 10 \%$ over the radiation exposure range 0 to $10 \mathrm{MRads}$. This measurement demonstrated that a) the dyes remain essentially undamaged and b) the light transfer from primary to secondary dye in the UV spectral range is not affected by any possible damage to the polymer at these wavelengths.

Since, we have not yet produced large plates we have not been able to determine the effect of scintillator light absorption by the plastic. However, we have measured the transmission spectra of the scintillator plate both before and after exposure to $10 \mathrm{MRad}$. This result is shown in figure $17 \mathrm{a}$. It can be seen that a) the secondary dye produces a sharp cut off in transmission at about $400 \mathrm{~nm}$ b) within experimental error there is no change in light transmission in the range above $400 \mathrm{~nm}$. We conclude from these measurement that plastic absorption of scintillator light probably should not be a significant problem. In the near future we should be able to excite the plastic plate in one location and measure the light output an inch or two distant to provide more definitive information on this subject.

We have not yet been able to make a measurement of light output of the low $T_{B}$ scintillator plate compared to standard material. We expect a 10-20\% lower light output due to the $10-20 \%$ by weight plasticizer material which does not contribute to light production.

The spectrum of scintillator light was measured after excitation at $320 \mathrm{~nm}$. This is shown in figure 17B for plastic both before and after an exposure of $10 \mathrm{Mrad}$. The spectra are normalized at their peaks. No significant change in the shape of the spectrum is observed. This is additional confirmation that the dyes are stable during the radiation, since 
there is a distortion of spectral shape 'due to dye degration.

Plate Production and Fiber Spinning:

Although this year the focus of the Detector Development Group was primarily on materials identification, we did allocate a block of time to initiate studies on the processing of fibers and plates

In a collaborative effort with Dr. Chang Park in the Chemical Engineering Department at the University of Florida, we spun prototype bicomponet (clad) fibers. Two types of fibers were spun:

a) unreactive siloxane fibers clad with fluorocarbon blends

b) polystyrene fibers clad with PMMA.

This important work demonstrated the ability to produce stable, bicomponent fibers whose core polymer could have a $T_{B}$ below or above room temperature. The next step in fiber production requires the use of equipment dedicated to optical fiber spinning and clean room facilities. U. of F. is negotiating to license patents for production work to industry.

We have produced scintillator plates by both casting and compression molding. A picture of a compression molded plate is shown in figure 18. This year we will continue plate production with a focus on injection molding.

Conclusions on Progress Made During 1990-1991

1. We have identified radiation hard, thermoplastic, heat processible polymers:

plasticized polystyrene

polyisobutyl methacrylate

PhFBM (cladding material) 
2. We have identified the following radiation hard fluors:

$$
\begin{array}{ll}
\text { primary fluor } & \text { oligo 374A } \\
\text { secondary fluor } & \text { TPB } \\
\text { wavelength shifters } & \text { Coumarin 545 }
\end{array}
$$

Fluorol 7GA 
TABLE 1

POLYMER $V \times 10^{5} \mathrm{M} / \mathrm{HR} \quad \mathrm{T}_{\mathrm{g}}(\mathrm{C}) \quad P \times 10^{11} \mathrm{~cm}^{2} / \mathrm{sec} \mathrm{ml} \mathrm{O} \cdot \mathrm{mm}$

PS

1.1

100

2.3

PMMA

0.01

105

0.3

PEMA

0.84

66

2.7

PiBMA

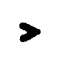

57

5.8

PPMA

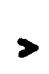

35

3.3 
TABLE 2

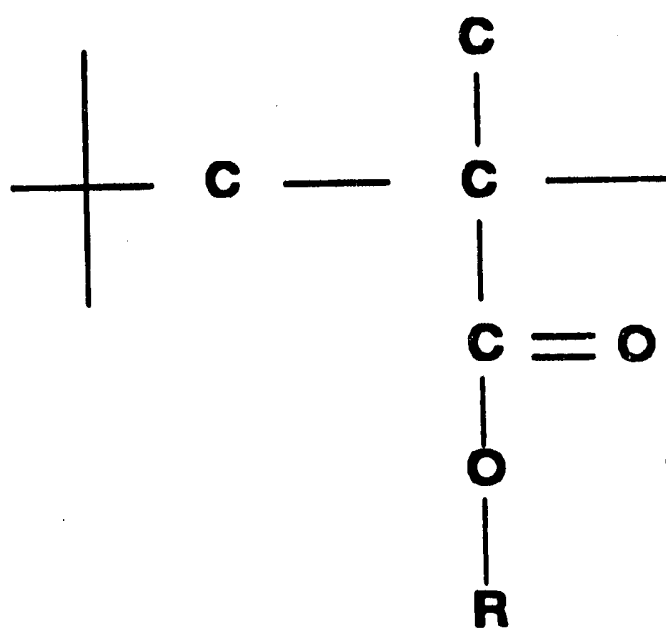

MONOMER

R

$T_{\sigma^{\prime}} \mathbf{C} d y n / C m$

Trifluoroethylmethacrylate

2,2,3,3-tetrafluoropropyl methacrylate

$-\mathrm{CH}_{2}-\mathrm{CF}_{2}-\mathrm{CF}_{2}-\mathrm{H}$

1H, $1 \mathrm{H}$-pentafluoropropyl

methacrylate

$-\mathrm{CH}_{2}-\mathrm{CF}_{2}-\mathrm{CF}_{3}$

70.4

38.37

Hexafluoroisopropyl

methacrylate

$-\mathrm{CH}_{2} \overbrace{\mathrm{CF}_{3}}^{\mathrm{CF}_{3}}$

1H, 1H-heptafluorobutyl methacrylate

$-\mathrm{CH}_{2}-\mathrm{CF}_{2}-\mathrm{CF}_{2}-\mathrm{CF}_{3}$

$1 \mathrm{H}, 1 \mathrm{H}, 5 \mathrm{H}$-octafluoropentyl methacrylate

$\mathrm{CH}_{2}-\mathrm{CF}_{2}-\mathrm{CF}_{2}-\mathrm{CF}_{2}-\mathrm{CF}_{2}-\mathrm{H}$

68.3

40.43

PMMA 

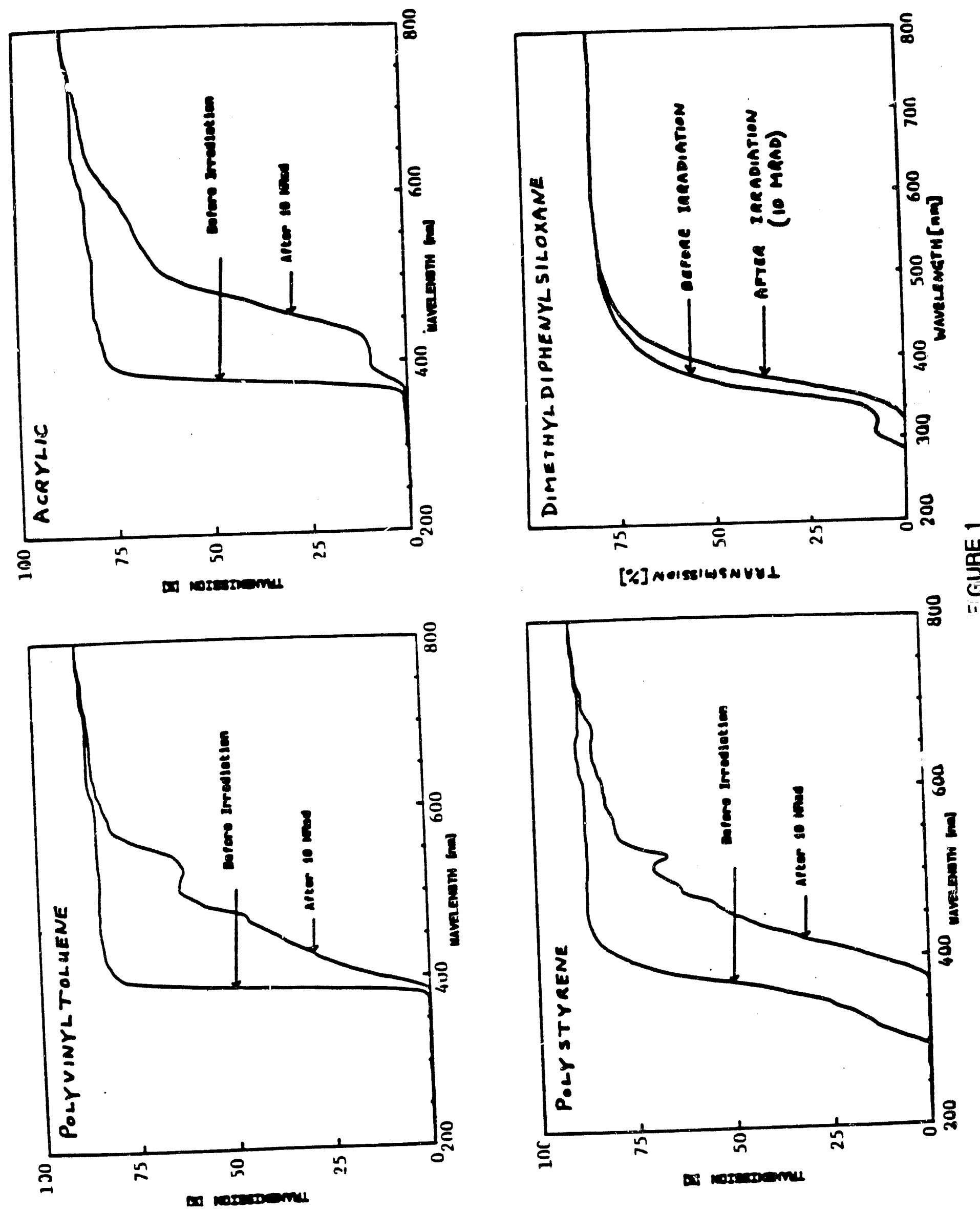


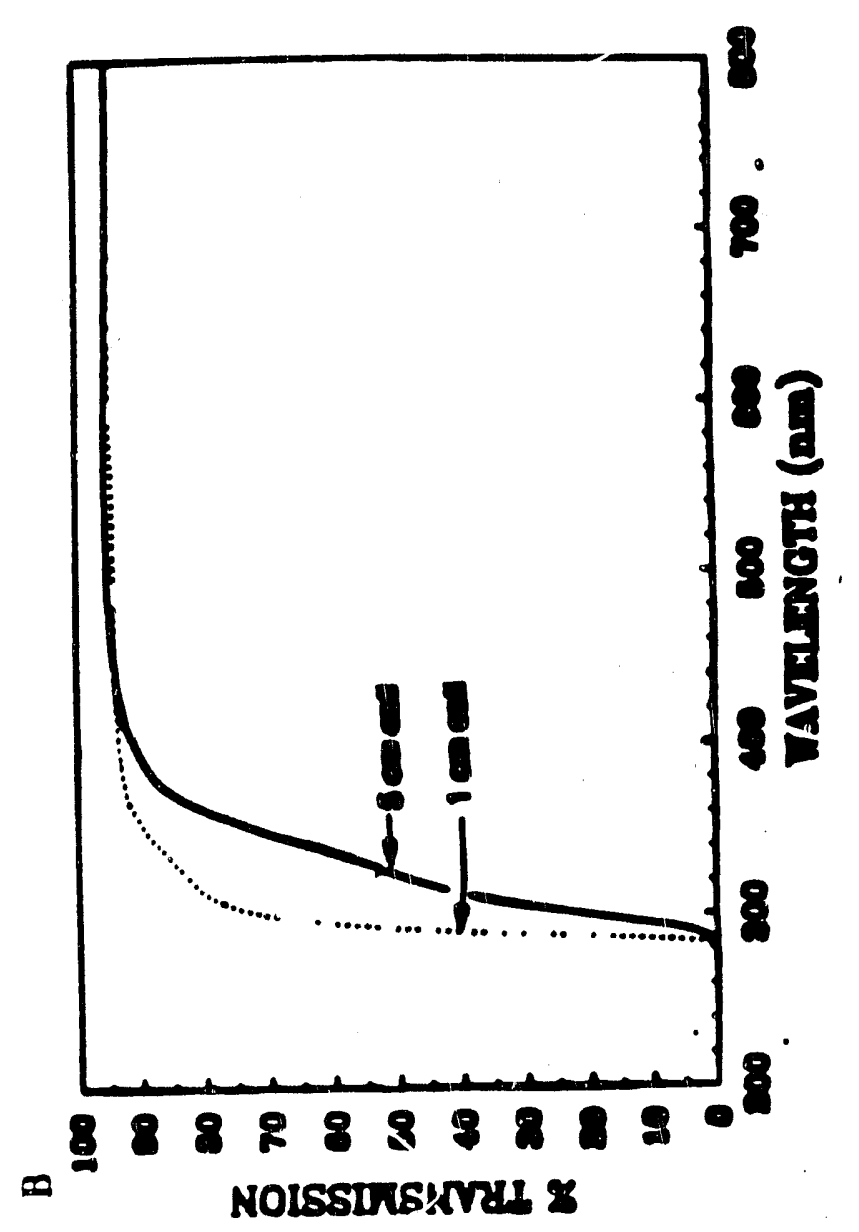

$\varepsilon 乙$
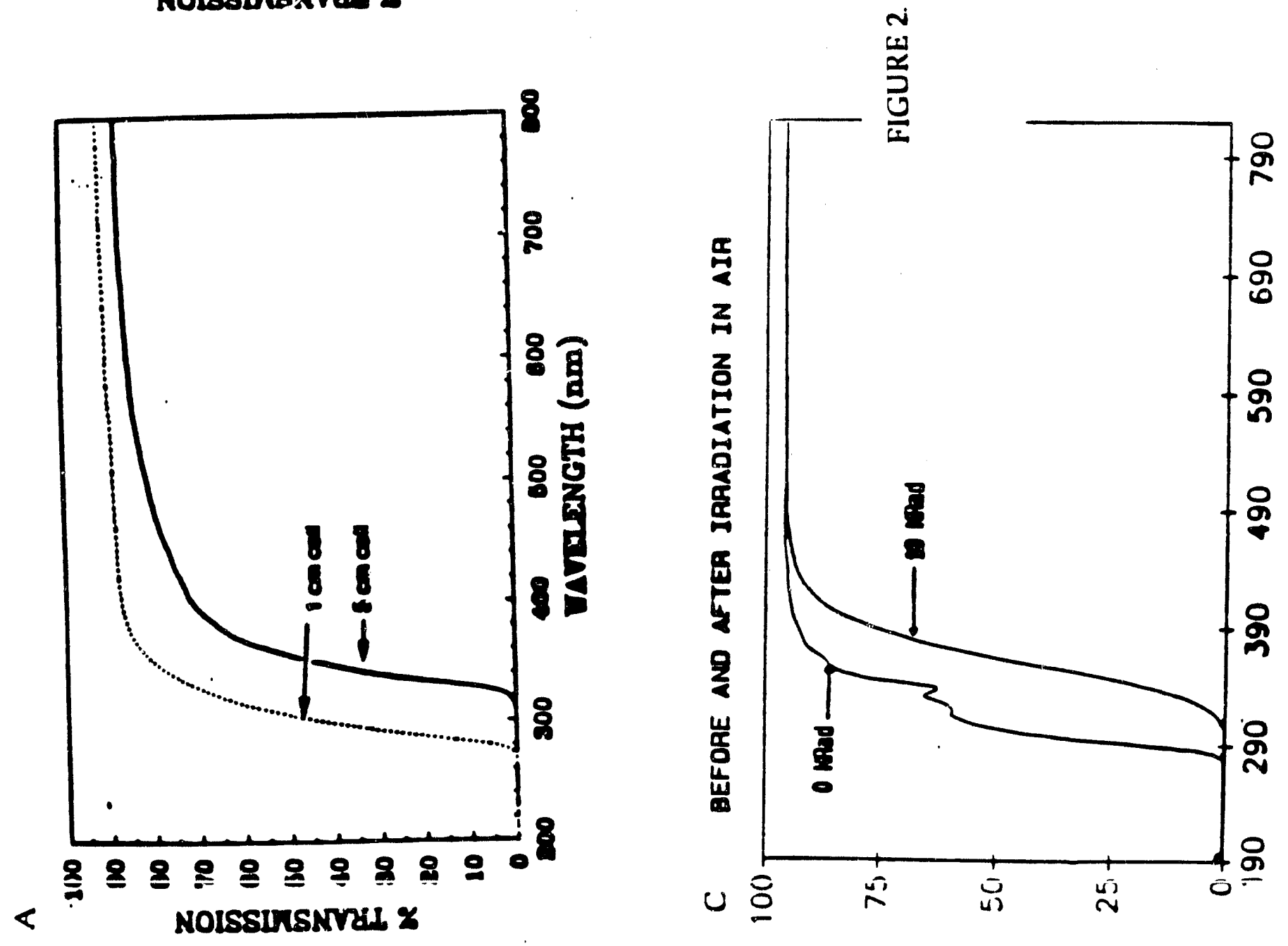


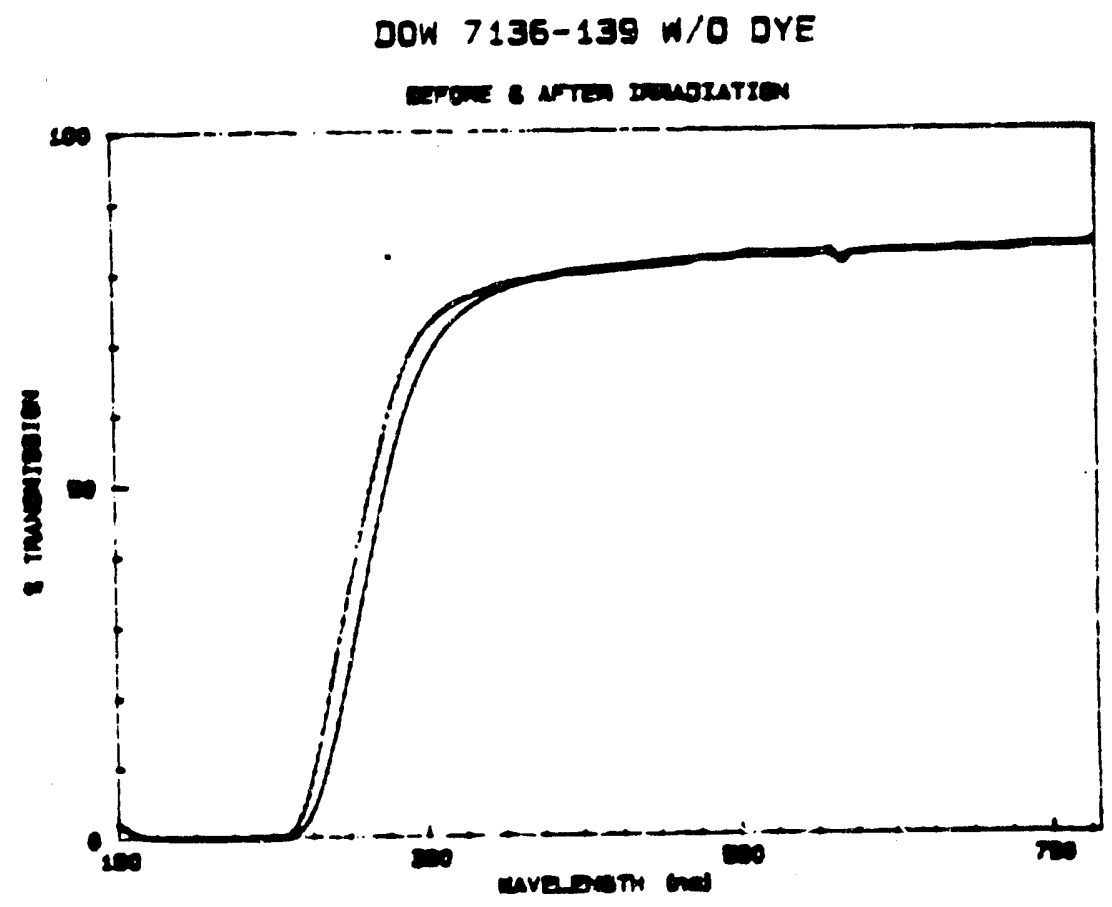

FIGURE 3. DOW CORNING PLATE BEFORE AND AFTER 10 MRAD 


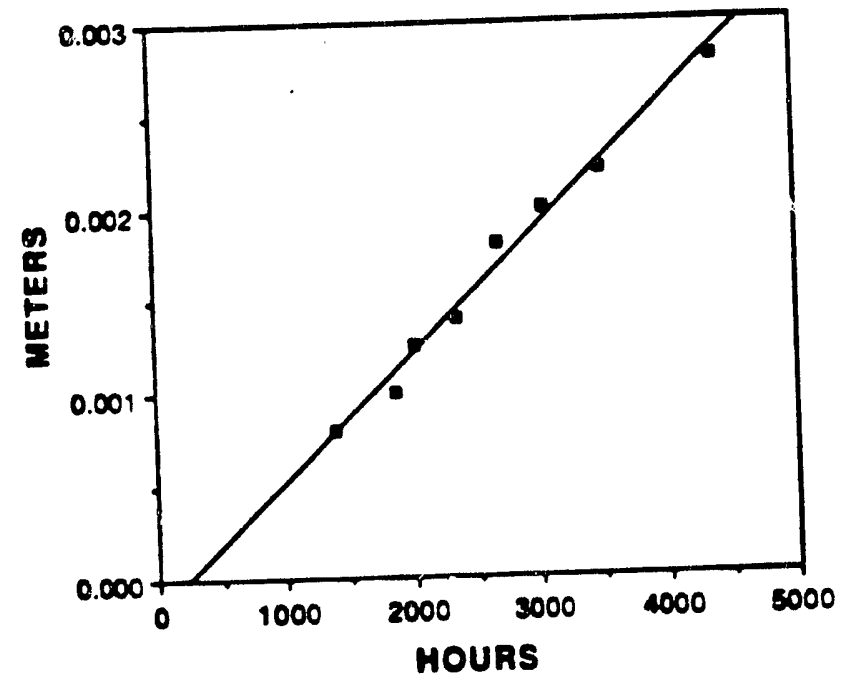

PMMA RECOVERY BOUNDARY MOVEMENT

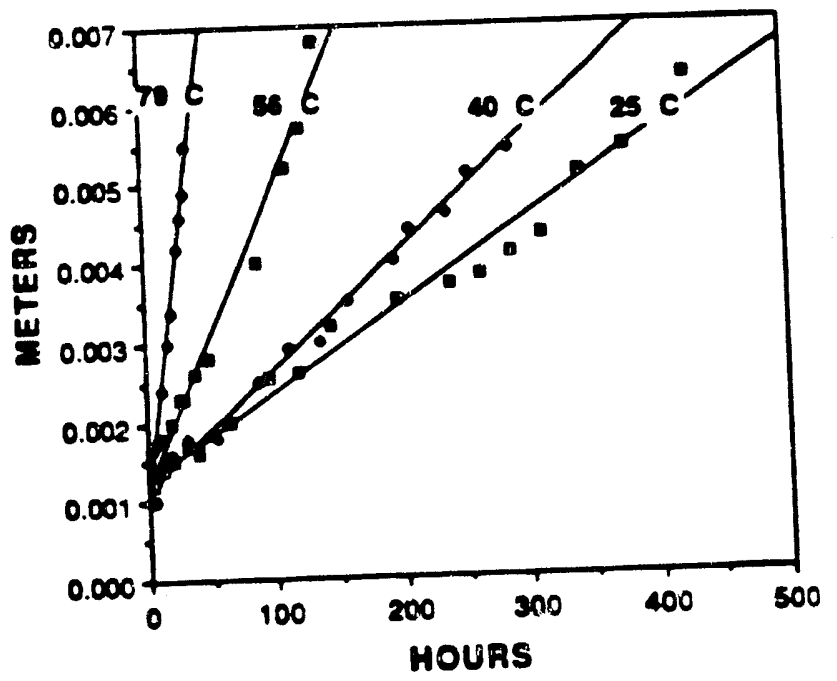

PS RECOVERY BOUMIDARY MOVEMENT

FIGURE 4. 


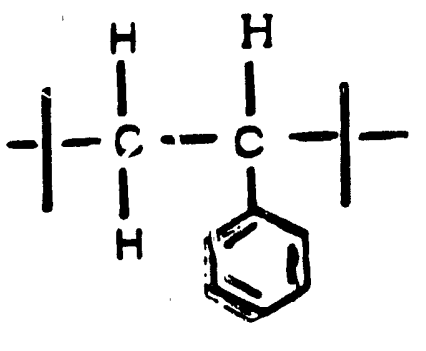

PS

$T_{8}=100 \mathrm{C}$

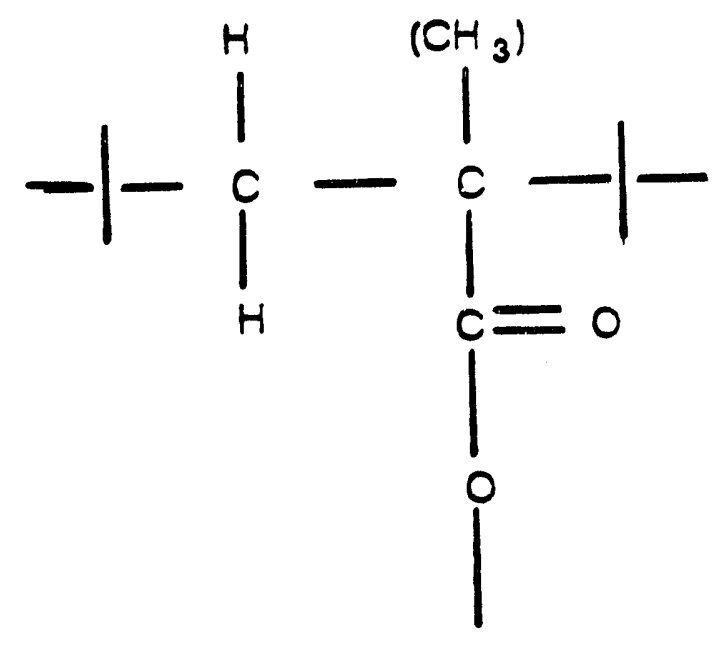

$\left(\mathrm{CH}_{3}\right)$

PMMA

$T_{g}=105 C$

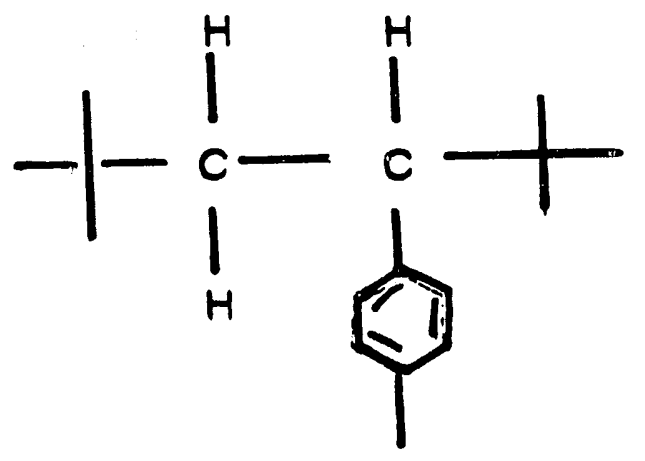

$$
\left.\right|_{\left(\mathrm{CH}_{3}\right)} ^{\left(\mathrm{CH}_{2}\right)_{9}}
$$

POLYDECYL STYRENE

$$
T_{8}=-65 C
$$

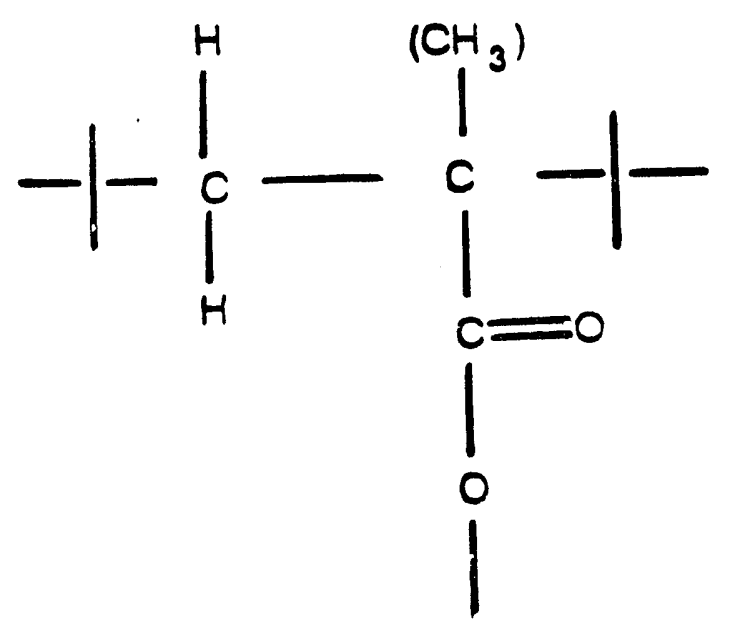

$\left(\mathrm{CH}_{2}\right)_{5}$

POLY (n-HEXYL METHACRYLATE)

$$
T_{8}=-5 C
$$

FIGURE 5. 

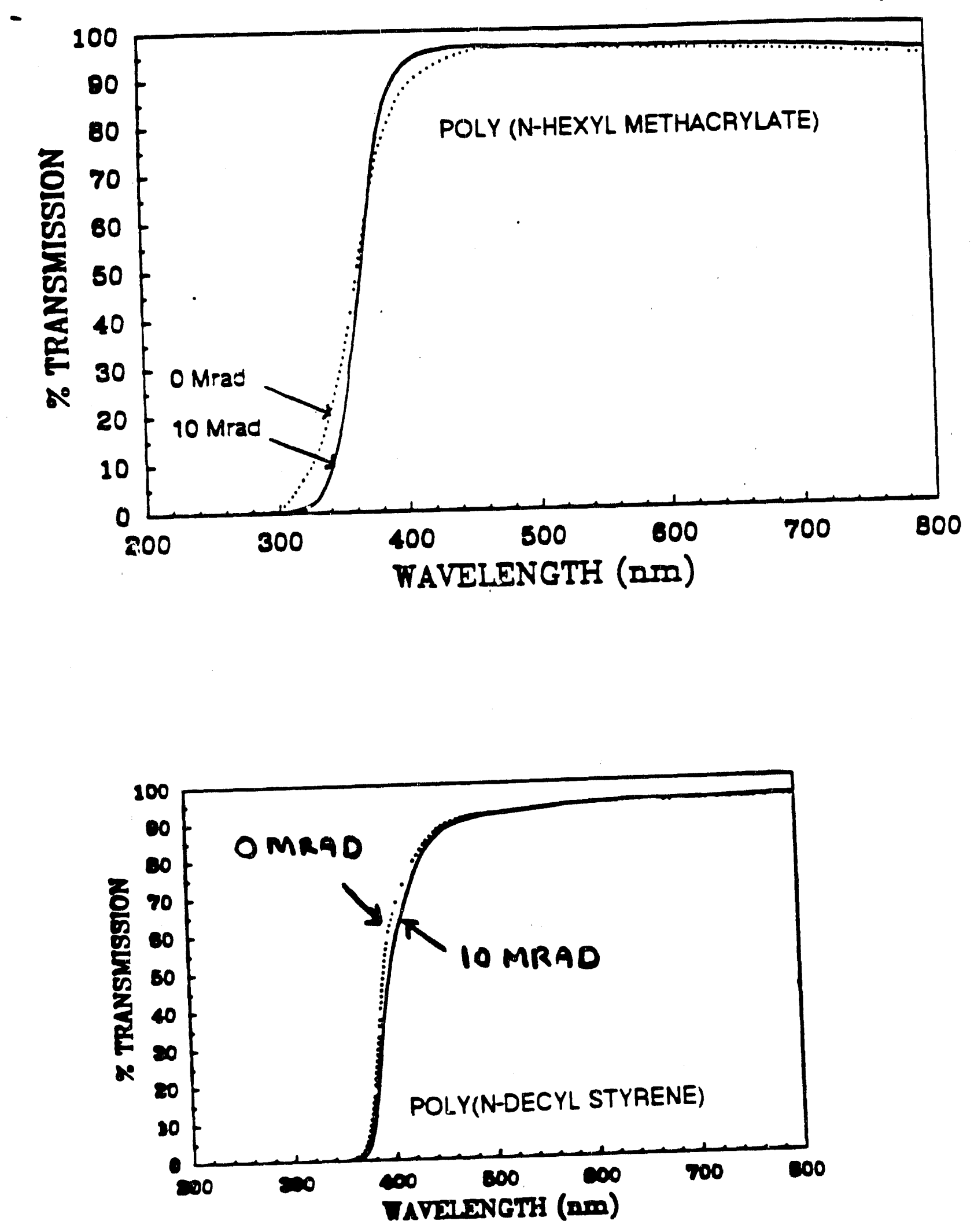

FIGURE 6. 


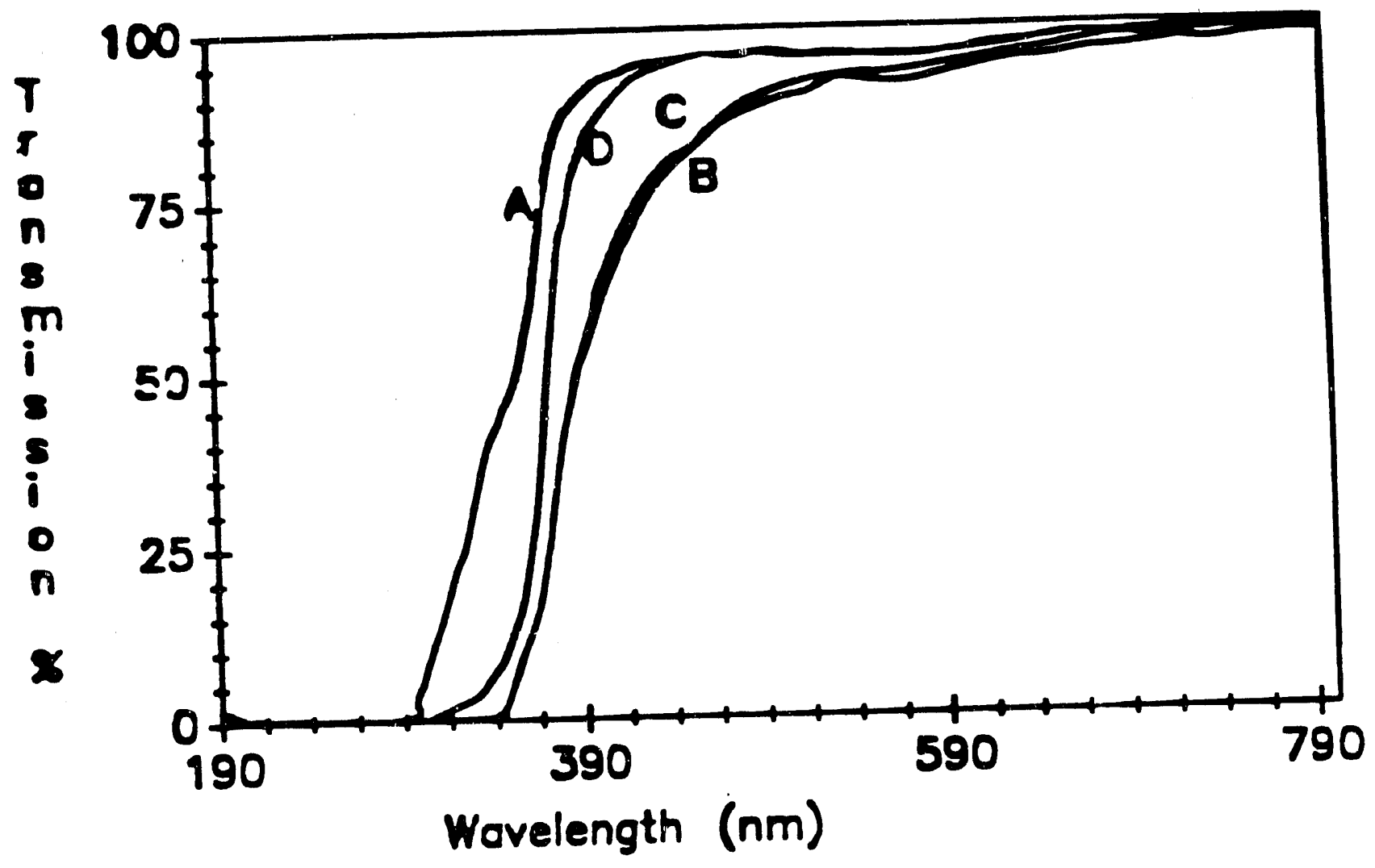

FIGURE 7. PS A BEFORE RADIATION

B AFTER 10 MRAD

C 3 DAY RECOVERY

D 30 DAY RECOVERY 


\section{EFFECT OF DOSE ON PS RECOVERY DORDER}

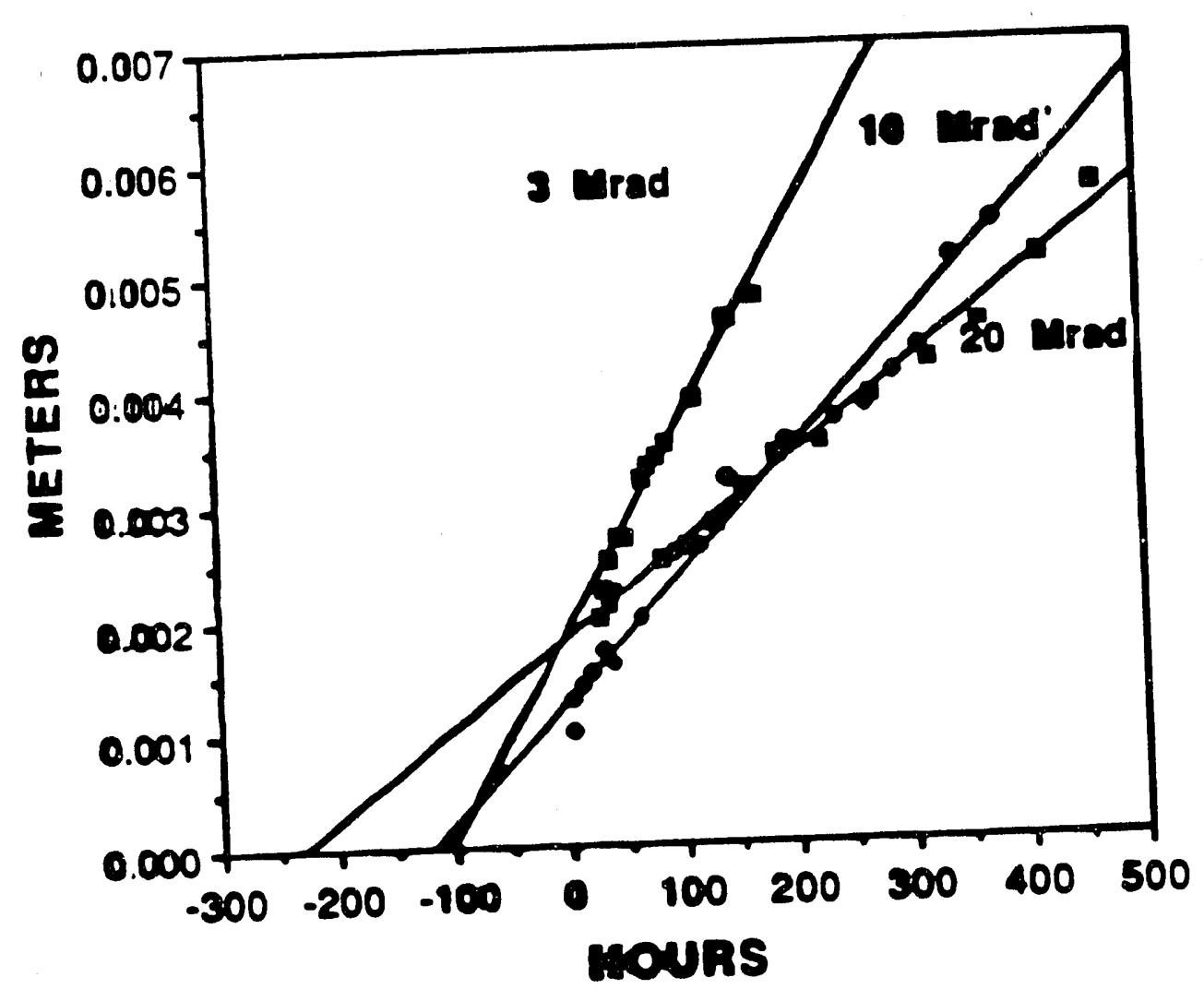

Dose

VELOCIN

3 MRad

$1.8 \times$ W.

10 Mrad

$1.1 \times 10^{-5}$ METERSAIOUR

20 Mrad

$0.79 \times 10^{-6}$ METERSHOUR

FIGURE 8 

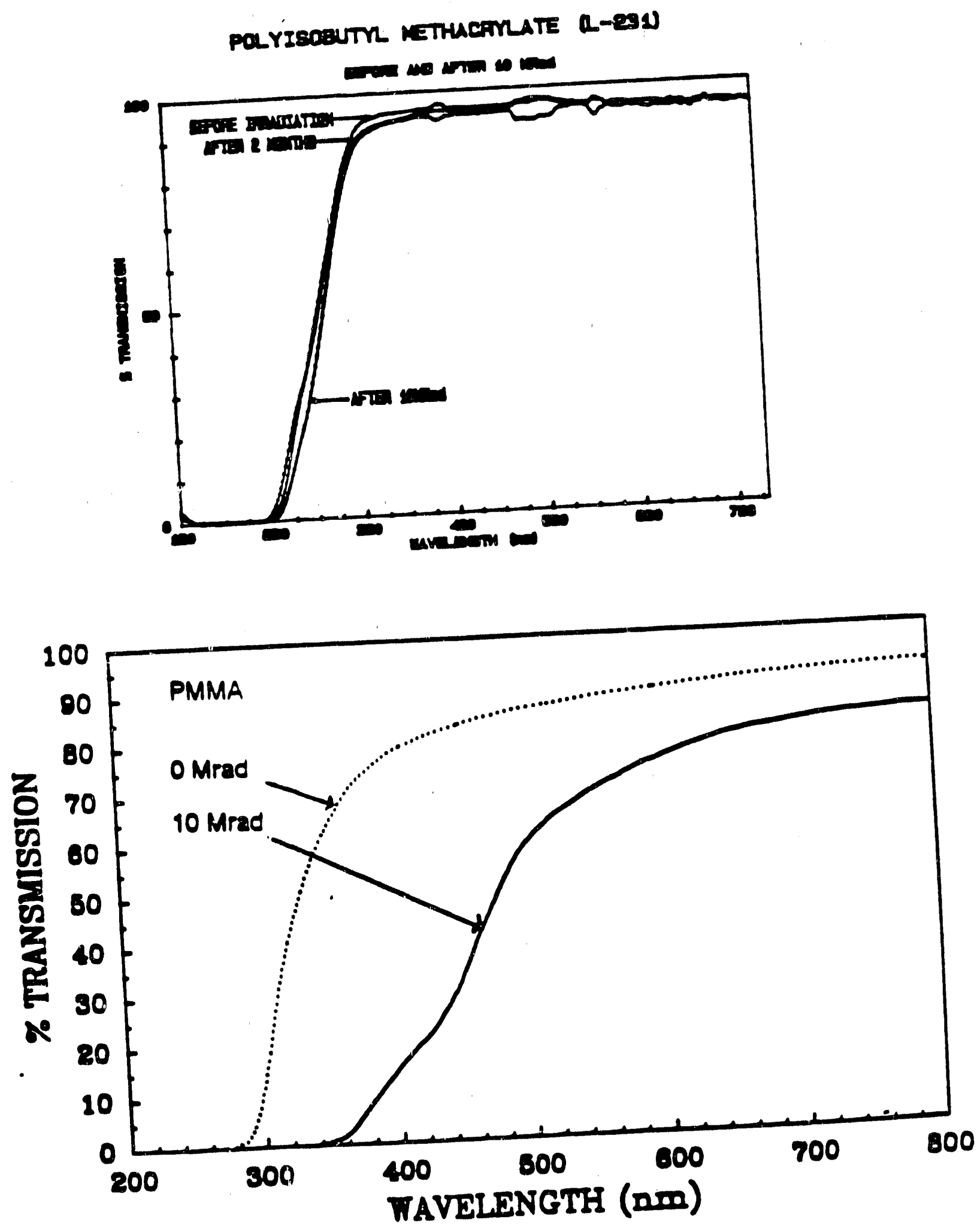

FIGURE 9 

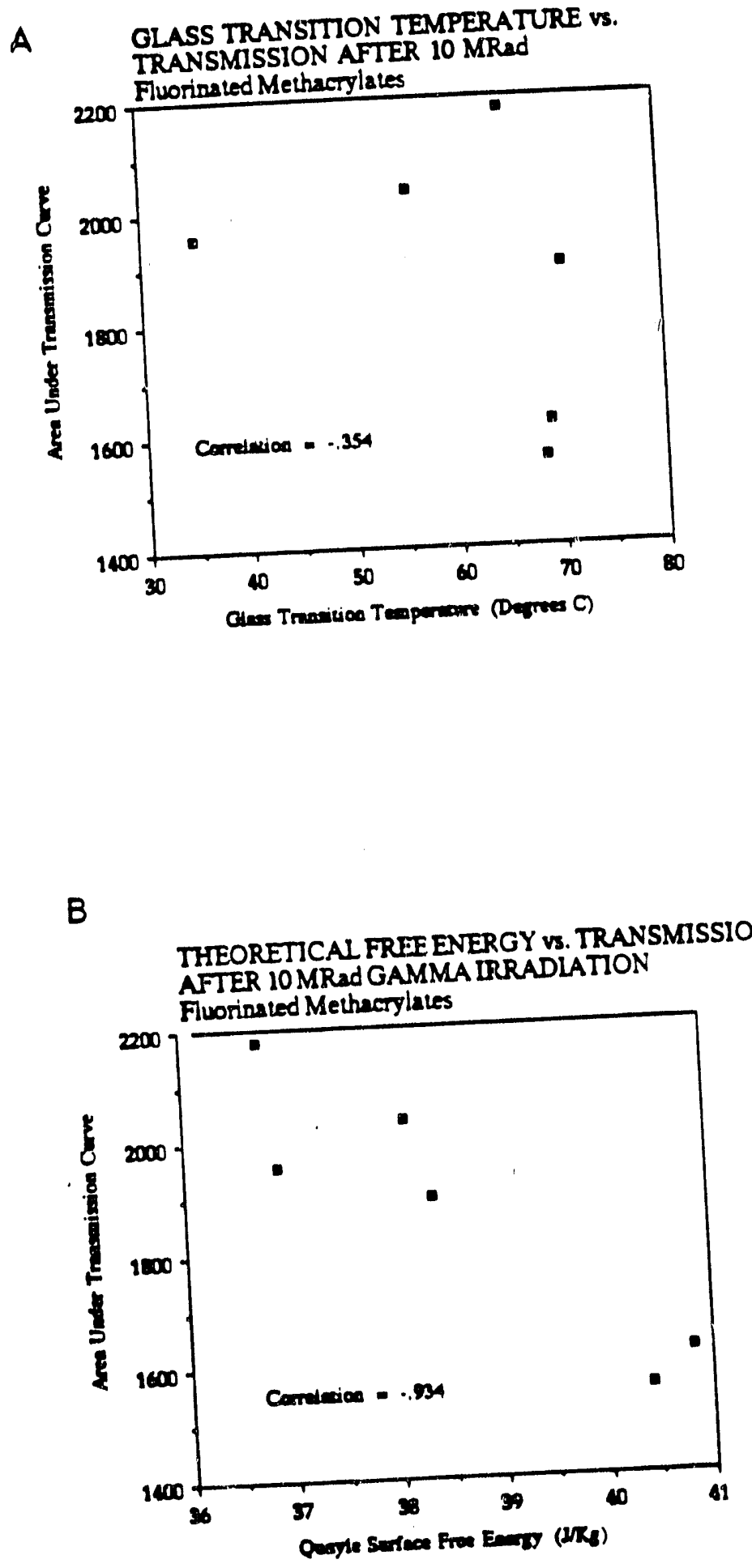

FIGURE 10 


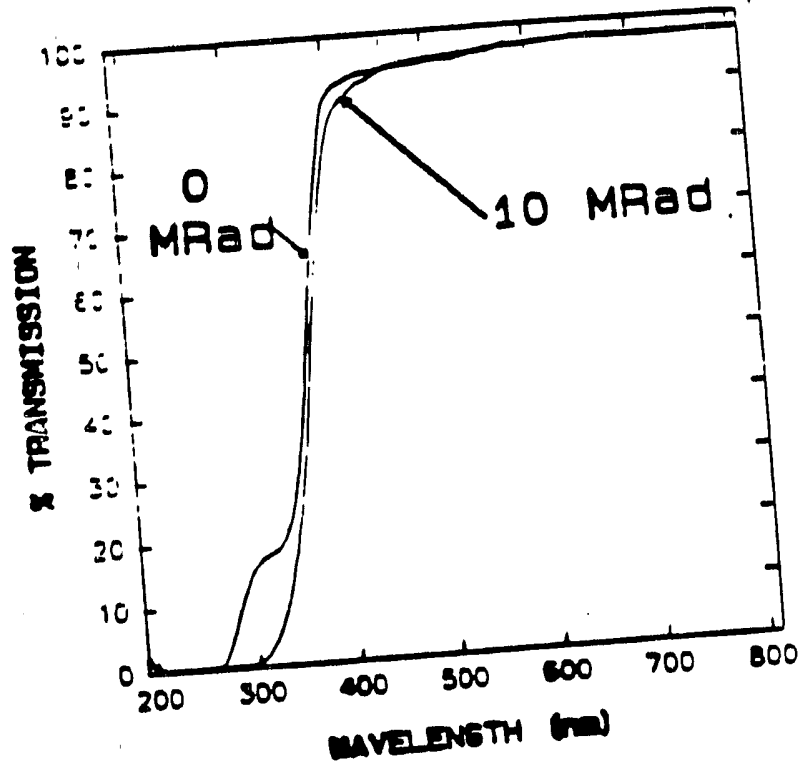

FIGURE 11. PHFBM CLADDING MATERIAL

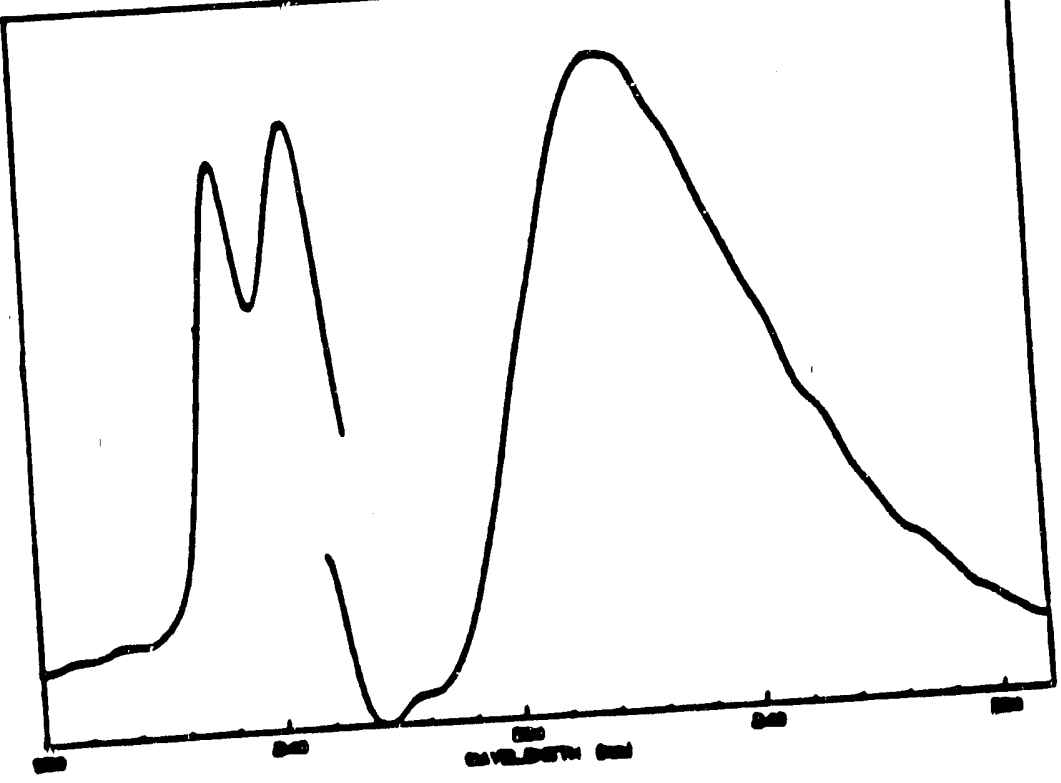

FIGURE 12. ABSORPTION - EMISSION OF PS 


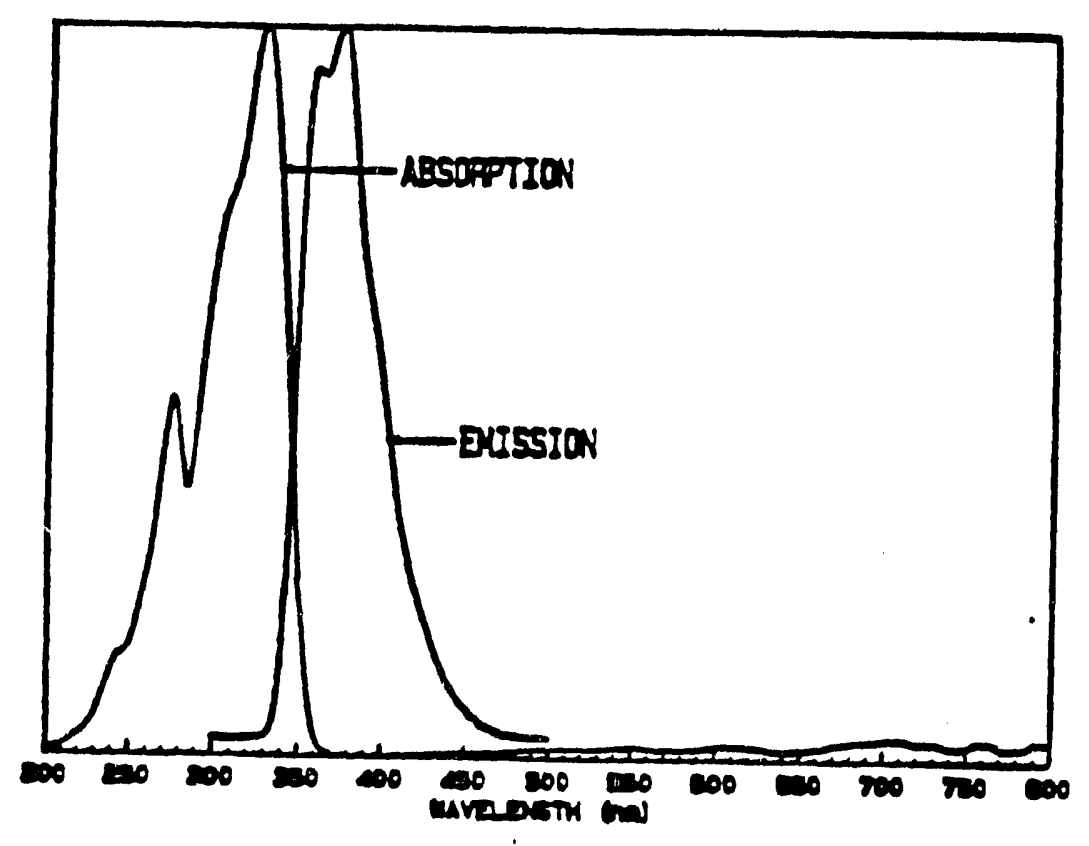

FIGURE 13. ABSORPTION - EMISSION OF OLIGO 374 A

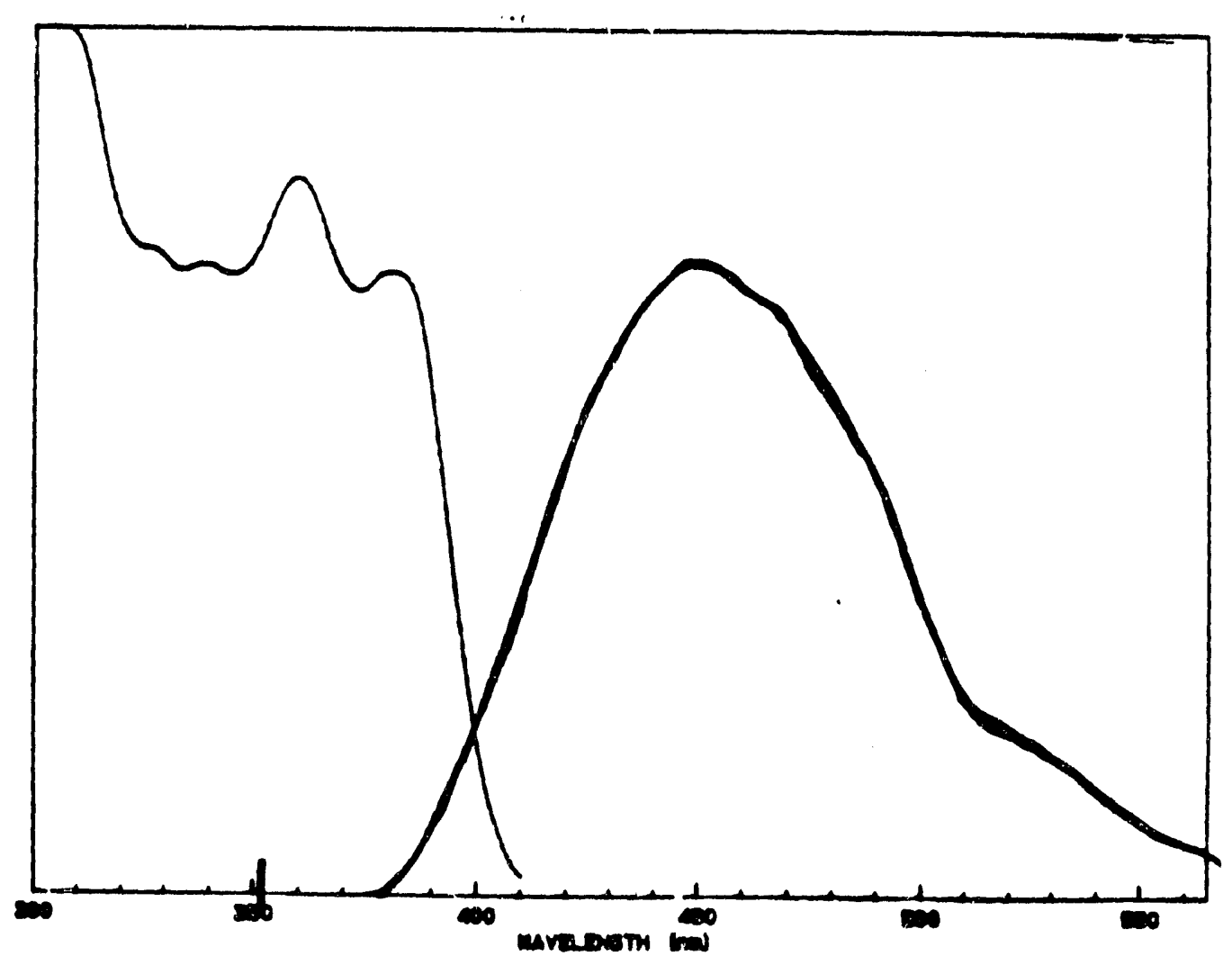

FIGURE 14. ABSORPTION-EMISSION OF TPB 


\section{RELATIVE LIGHT OUTPUT}

indiction in arven

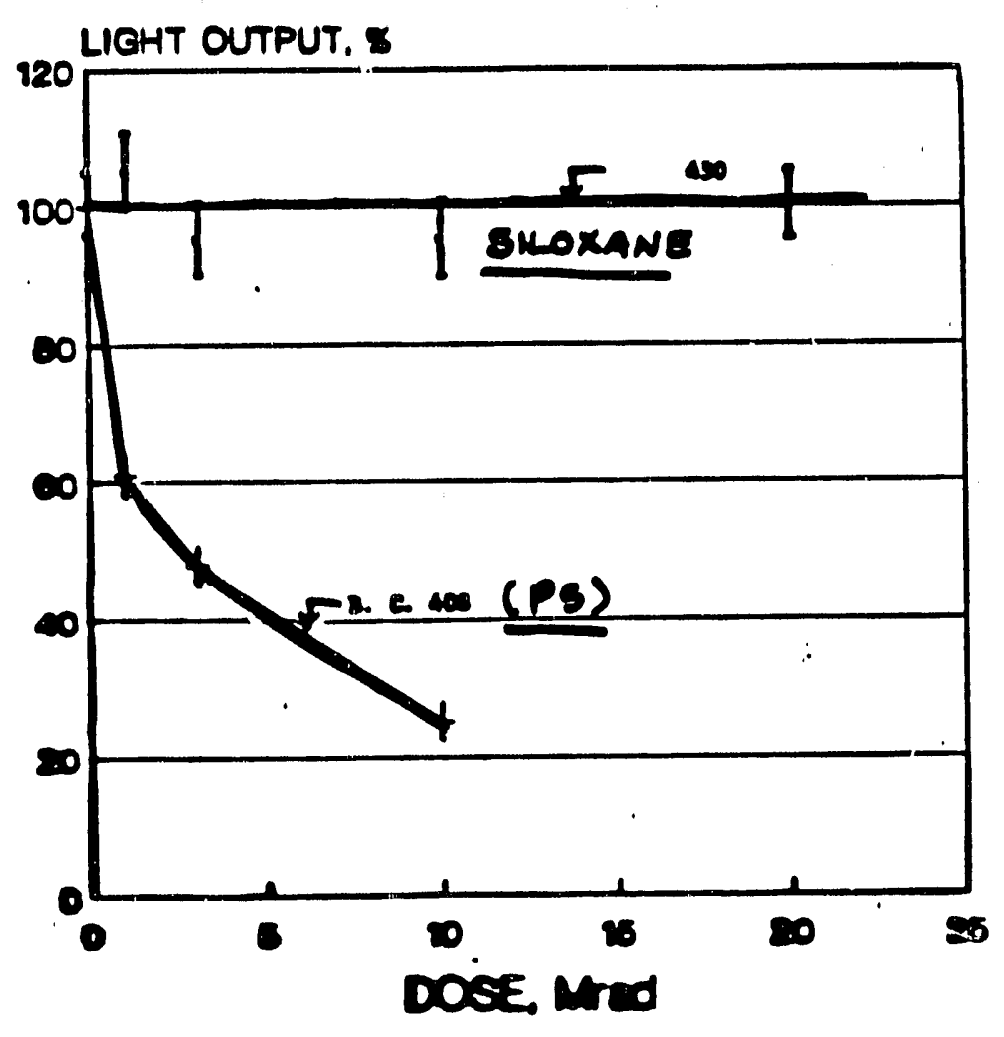

FIGURE 15. 


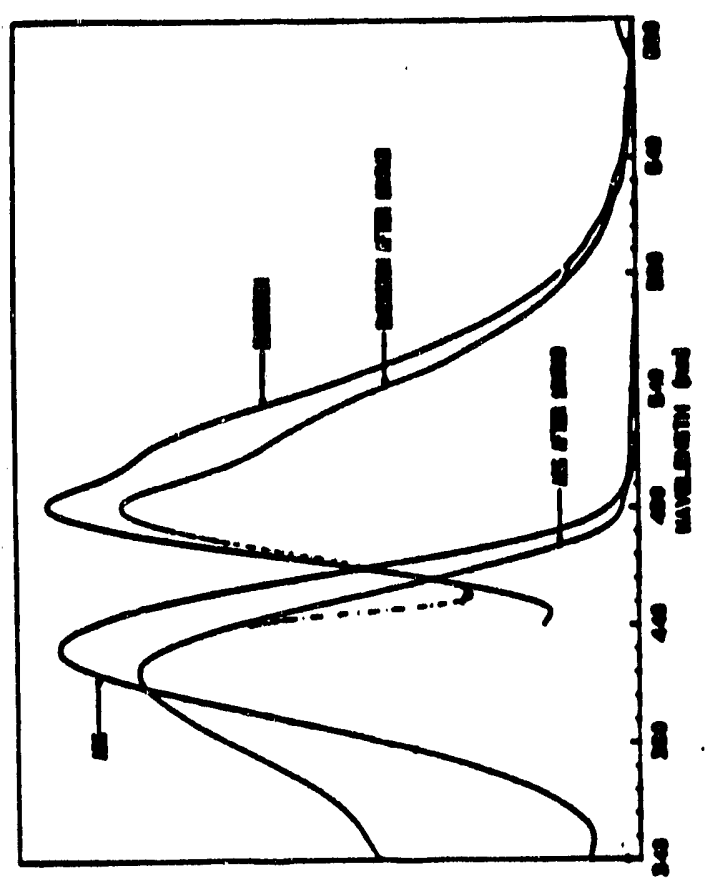

$\infty$

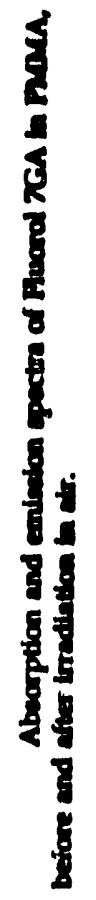

$\frac{\stackrel{0}{0}}{\frac{w}{5}}$
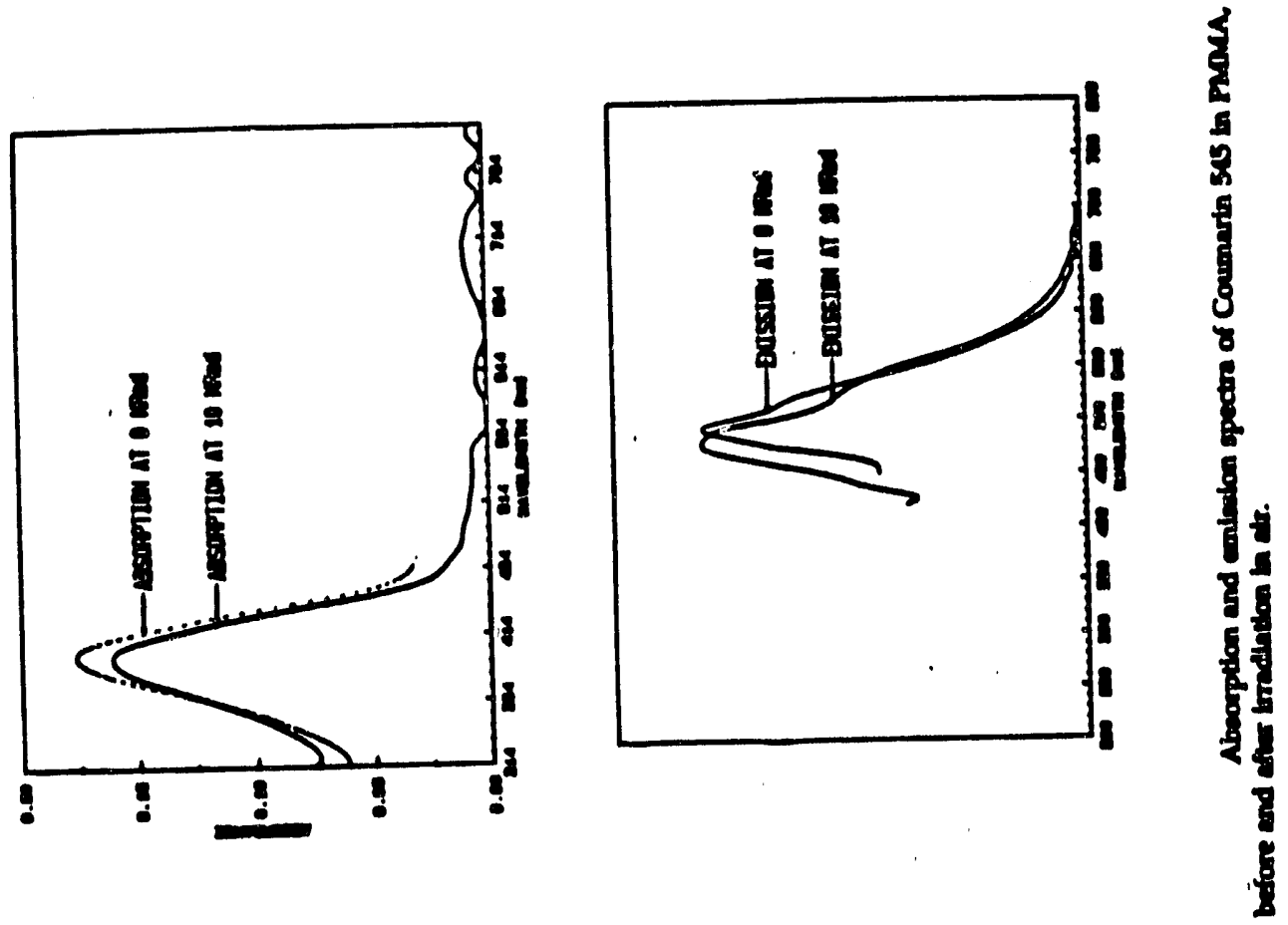
PS WITH 18\% DIBUTYLPHTHALATE, 1\% 0IIgO $374 \mathrm{~A}, 0.1 \%$ TPB BEFDAE AND AFTER 10 MAAJ
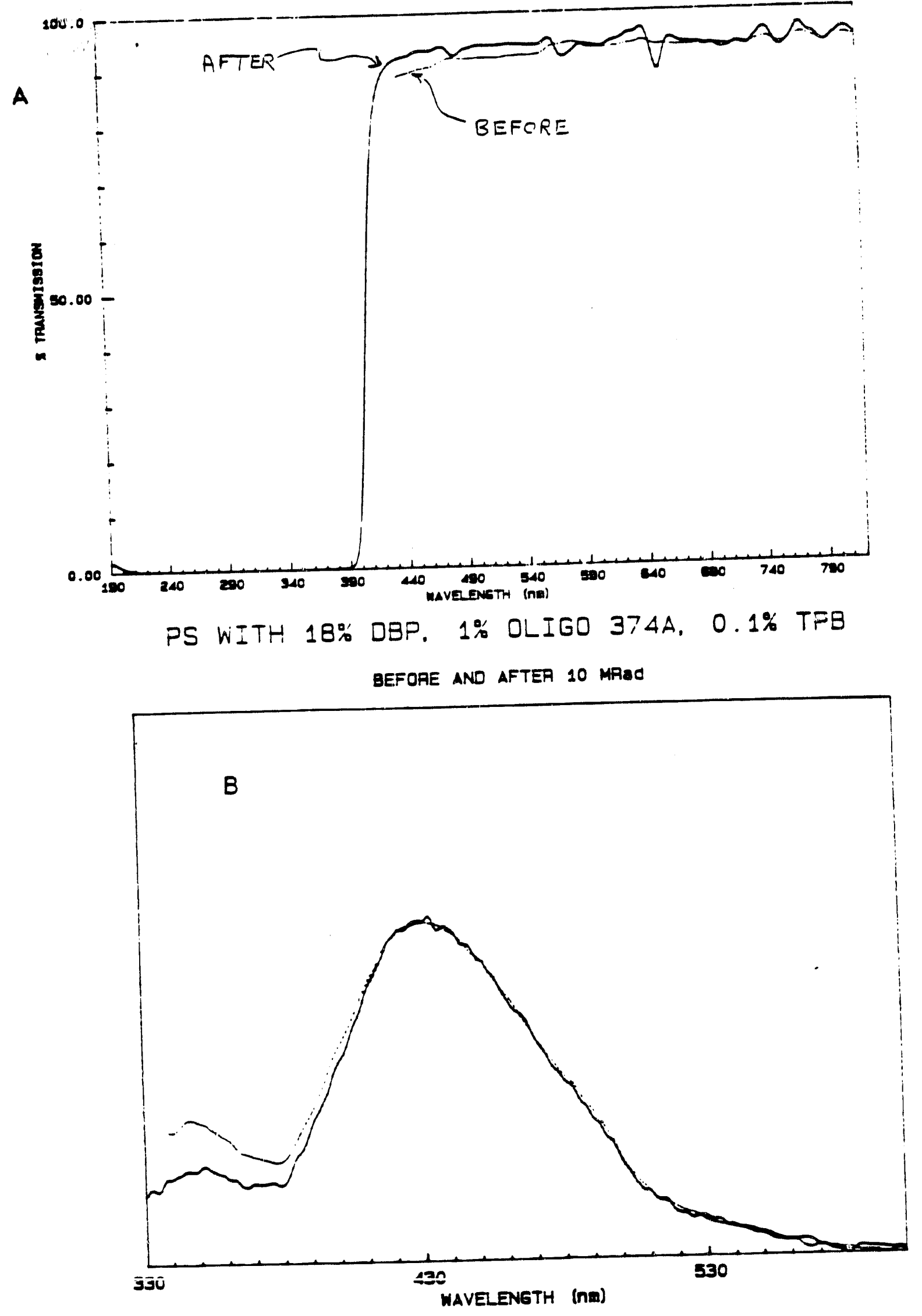

FIGURE 17 


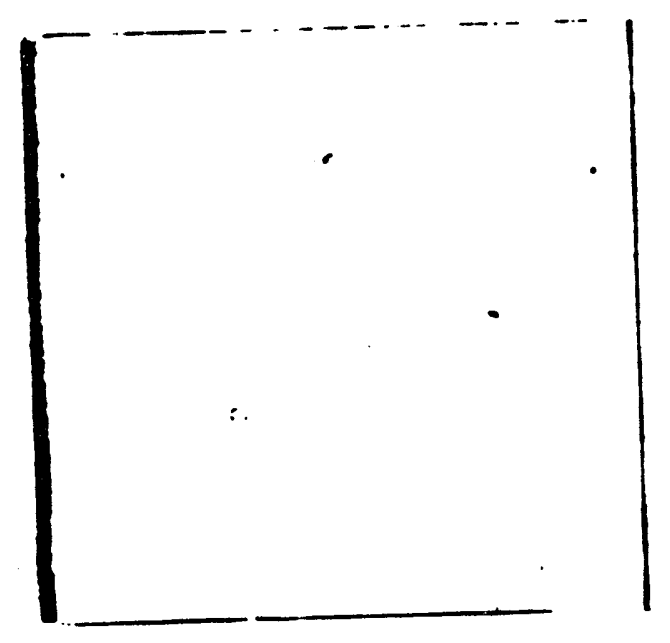

FIGURE 18 COMPRESSION MOLDED PLATE OF PS 


\section{References:}

1. S. E. Miller, A. G. Chynoweth, "Optical Fiber Telecommunications", New York, Academic Press, 1979, p. 354

2. D. W. Van Krevelen, "Properties of Polymers", Elsevier Publishing Co., New York, 1976 , p. $129-155$.

3. J. Harmon, PhD Thesis, "Diffusion in Deformed PMMA", University of Rochester, 1983.

4. J. Harmon, S. Lee and J. C. M. Li, "Polymer", Vol. 29, 1221-1226 (1988).

5. J. Harmon, S. Lee, and J. C. M. Li, "J. Polymer Sci.: Part A: Polym. Chem.", Vol. 25, 3215-3229 (1987).

6. Roger Clough, Sandia National Laboratory, private communication.

7. University of Florida, Julie P. Harmon and James K. Walker, Patent application 0805.

8. University of Florida, Julie P. Harmon, Patent application 0857.

9. L. Zlatkevich, "Luminescence Techniques in Solid State Polymer Research", New York, Marlel Dekker, 1989.

10. Ref. 2, page 161

11. V. Feygelman, J. Walker, J. Harmon, "Nucl. Inst. and Methods", A290 (1990) 131 135.

12. V. Feygelman, J. Walker, J. Harmon, "Nucl. Inst. and Methods", A295 (1990) 94-98.

13. Ami Jhaveri, Master's Thesis, University of Florida, "Polymer-Dye Compositions for Improved Scintillation Counters", 1991.

14. J. Harmon and J. Beatty, "Engineering Plastics, Engineered Materials Handbook", Vol. 2, ASM, 1987, 761-760. 


\section{Work Plan for 1991-1992}

We have id nuified radiation hard fluors and thermoplastic polymers. We have now moved into an area of research which focusses on accelerated life testing these materials and materials obtained from uther members of the SSC community. We have organized an experimental program which we present here. This program includes the production of scintillator with low $T_{8}$ thermoplastic polymers that are described in this proposal, life testing of these materials, life testing of materials from the SSC community and an experimental program to injection mold scintillator plates. This program is concerned with optimizing injection molding conditions to minimize residual stresses and testing the effect of residual stresses on radiation damage. This proposed work is broken down into the four tasks:

Task I Producing Polymers and Scintillator

Task II Life Testing Radiation Hard Materials Produced by the Detector Development Group at the $U$ of $F$

Task III Life Testing Materials From Other SSC Groups

Task IV Study of Injection Molding of Scintillator

A discussion of each of these tasks follows. 


\section{TASK I}

\section{Producing Polymers and Scintillator}

\section{Table of Contents}

1. Scope of the Task

2. Compounding

3. Polymerizing Scintillator and Clear Fiber Materials

4. Budget

5. Budget Justification

6. Time Schedule 


\section{Scope of Task I}

The primary purpose of this task is to produce material for injection molding studies. Cladding and fiber materials will also be made.

This task will also identify the most cost effective efficient way to produce materials.

The following materials will be produced by the Detector Development Group at the University of Florida:
a) PS plasticized with dibutylphthalate
b) PS plasticized with bibenzyl
c) poly (isobutyl methacrylate) (PiBM)
d) poly (heptafluorobutyl methacrylate) (PhFBM) (cladding material)
e) a, b and $\mathrm{c}$ doped with $1 \%$ oligo $374 \mathrm{~A}$ and $0.1 \%$ TPB for scintillator plates
f) a, b and c doped with Coumarin 545 or Fluorol 7GA for wavelength shifter fibers

PS and PiBM are commercially available in high purity grades. Dow Chemical manufactures optical fiber grade PS. Scientific Polymer products manufactures high purity PiBM. There are two options for producing doped material with these polymers. The first option is to dope the commercially available polymers with plasticizer and dyes by compounding (mixing the molten polymer with dopants). The second option is to dissolve the dopants in the monomer and polymerize the dopants in situ. For the PhFMB cladding material, synthesis is the only option, since the polymer is not commercially available. This experimental program encompasses both compounding and synthesis. After material evaluation the most cost effective method will be chosen. 


\section{Compounding Study}

\section{a) Experiment}

The experiments proposed here require a variety of doped materials. It is necessary to compound with a device that is readily and completely cleaned after each material. We selected a Farrel Two Roll Mill for this reason. This device has two closely spaced rolls running at different speeds to provide a shearing action as the mix passes through the nip between the two rolls. The mill has two rolls 3 " diameter by 7 "long. It is driven by a $11 / 2$ HP gearhead motor and is electrically heated with controls.

b) Compounding Procedure

The temperature controls on the rolls are set at $80^{\circ} \mathrm{C}$ above the estimated glass transition temperature of the plasticized mixture. (The theoretical curves for PS plasticized with bibenzyle and dibutylephthalate are shown in figure 1. These are calculated as described in ref. 2.) The batch size for the Farrel Mill is $300 \mathrm{gm}$. The polymer, in the form of pellets, is dry mixed with dyes and plasticizer in polyethylene bags. The mixture is dropped onto the roll mill and compounded for 20 minutes. A doctor blade is used to remove the mixture from the rolls.

\section{c) Grinding}

When the compounded mixture is cooled, it is broken into chips and fed into a Wiley Mill. This results in a course ground material which is easily conveyed in the injection molder.

3. Polymerizing Scintillator and Clear Fiber Materials.

Scintillator and wavelength shifter will be produced by adding the appropriate dyes to 
the monomer before polymerization. (1\% oligo 374 and $0.1 \%$ TPB for scintillator and $0.1 \%$ Coumarin 545 or Fluoral 7GA for wavelength shifter)

$500 \mathrm{gm}$ batches will be synthesized according to the following procedure:

a) Inhibitors are removed from the monomer by passing it through an inhibitor removal column obtained from Scientific Polymer Products.

b) $\quad 0.1 \%$ L231 or BPO catalyst is added to the monomer and plasticizer while stirring under argon.

c) The mixture is poured into polyethylene tubes, sealed and polymerized in a water bath at $85^{\circ} \mathrm{C}$ for 6 hours, caps are removed, tubes are placed in an oven at $110^{\circ} \mathrm{C}$ for 4 hours to post cure.

d) The polymers are removed from the tubes broken into course pieces and fed into the Wiley Mill for grinding. 

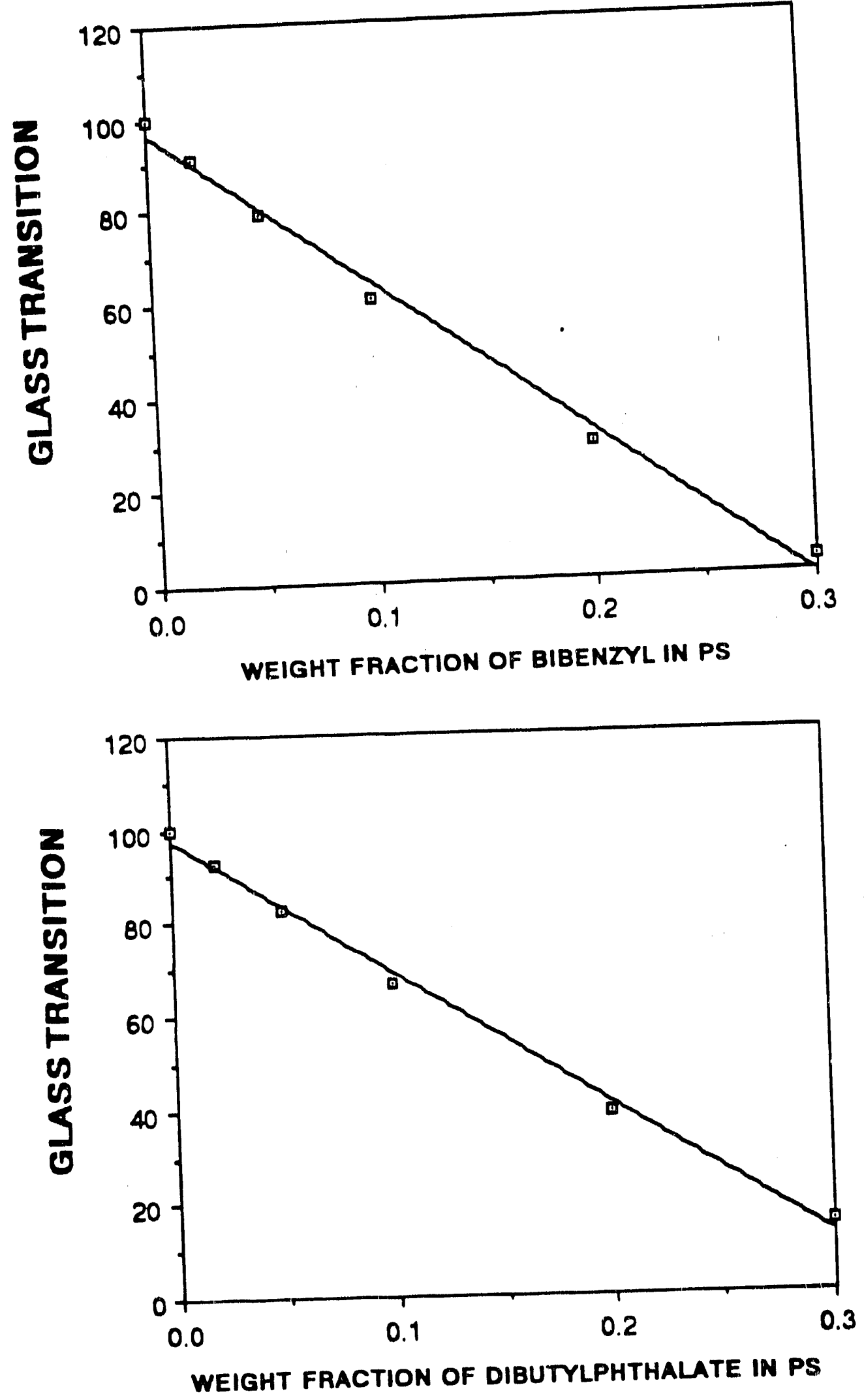

FIGURE 1 


\section{Time Schedule for Task I}

Purchase of equipment and installation: 1 month

Compounding: upon equipment installation material will be produced as needed

Polymerization: polymerization will begin at the notification of grant acceptance and continue throughout plate production. Cladding and fiber materials will be produced upon request. 


\section{TASK II}

Life Testing Radiation Hard Materials Produced by the Detector Development Group

\section{Table of Contents}

1. Scope of the Work

2. Physical Testing

3. Budget

4. Budget Justification

5. Time Schedule 


\section{Scope of Task II}

Physical properties of polymer and scintillator materials will be measured before and after radiation. We propose using polymers and scintillator with glass transition ternperatures lower than that of commercial scintillator. Polystyrene, for example, has a $T_{8}$ of $100^{\circ} \mathrm{C}$. Our low $T_{B}$ polymers, especially plasticized polystyrene exhibit increased flexibility compared to high $T_{2}$ polymers. This enhanced flexibility should enhance the bend strength of fibers. In addition, the plasticizer decreases susceptibility to radiation induced stresses. The more flexible polymer matrix should exhibit less susceptibility to stress induced crazing. This is probably an important characteristic for the tightly bent wave-length shifting fibers.

Radiation damage and recovery are affected by the radiation dose and dose rate.

We will characterize physical properties before and after exposures at a series of doses and dose rates. The irradiation source is ${ }^{60} \mathrm{Co}$. All radiation tests will be performed in air. Samples will be exposed to doses of 1,5 and $10 \mathrm{Mrad}$. The dose rates will be:

$$
\begin{gathered}
\mathrm{MR} / \mathrm{hr} \\
0.002 \\
0.018 \\
0.040 \\
0.570
\end{gathered}
$$

\section{Physical Property Measurement}

The following physical properties will be measured before and after irradiation:

a) Spectrophotometry 
In the past we have documented optical radiation hardness in studies conducted at dose rates of $3 \mathrm{MRad} / \mathrm{day}$. The effect of dose rate on optical properties will be monitored to verify that our materials are radiation hard at low dose rates. In tests at low do rates oxygen is less likely to be depleted. The presence of oxygen may influence reactions leading to color center formation. Transmission spectra will be measured on compression and injection molded samples with a Hewlett Packard 8452A diode spectrophotometer. Spectra will be recorded before and after irradiation at a series of doses and dose rates.

\section{b) Scintillator Light Output}

Light output studies will be done in several steps. The light output from injection molded plates will be measured using a ${ }^{207} \mathrm{Bi}$ source located 2" laterally from an RCA 8850 phototube. This distance simulates the typical distance that lights has to go before being absorbed by a wavelength shifting fiber. These measurements will be performed as a function of a variety of parameters such as conditions of injection molding, as described later, radiation dose, accelerated aging, etc.

Wavelength shifting fibers will be installed in the injection molded plates. Light output from these fibers will be measured in a manner similar to that described above.

We have constructed a $3 \mathrm{~m}$ long fiber testing set up. Light is produced in the fiber with an X-ray generator whose position is computer controlled. Attenuation lengths of fiber are obtained immediately.

c) Emission Spectra

We have determined that the fluors which we recommend are stable at doses up to 10 MRad given at a dose rate of $3 \mathrm{MRad} / \mathrm{day}$. Oxygen diffusion and chemical reactions which 
are induced by radiation may produce different effects at dose rates slower than $3 \mathrm{MRad} /$ day. Therefore, emission spectra will be monitored throughout life testing on a Hewlett-Packard Model 1046A Programmable Fluorescence Detector.

d) Molecular weight

Radiation induces scission and cross-linking reactions. We will monitor the extent of these reactions based on changes in molecular weight. Scission and cross-linking reactions locally expand and contract the polymer matrix. These reactions are responsible for radiation induced crazing. It is extremely important to monitor the effect of dose rate on these reactions. This will aid in predicting long term scintillator stability. A gel permeation chromatograph will be used to characterize molecular weight. Samples will be dissolved in chloroform. The molecular weights characterized will be based on polystyrene equivalent molecular weights. This instrument separates the components (dyes, plasticizers) and analyzes molecular weights of the different fractions. Cross-linked components will be filtered from solutions in a 0.2 micron filter prior to GPC analysis.

e) Flexural modulus

The effect of radiation induced reactions on the flexural modulus is an important feature that has not yet been tested in scintillator. In addition, the plasticized polymers that we are studying here have lower flexural moduli than commercial scintillator. These systems must be characterized.

A Dupont 2100 dynamic mechanical analyzer will be used to monitor changes in modulus versus temperature and to characterize the glass transition temperature. This instrument deforms $3 \mathrm{~mm} \times 10 \mathrm{~mm} \times 2 \mathrm{~mm}$ samples in the flexural mode at a series of 
frequencies. The plasticized PS that we are studying here has a lower flexural modulus than commercial scintillator.

\section{f) Stress Relaxation}

Iradiation reactions induce stress in polymeric scintillator. For years, it has been known that scintillator often cracks upon exposure to irradiation. We have shown that the ability to dissipate stress, or relax, imparts the polymer matrix with optical radiation hardness. As discussed in 'uis introduction, plasticized polymers and polymers with reduced $T_{8}$ 's are more optically radiation hard. We will use mechanical stress relaxation to predict the effect of radiation induced stresses on scintillator. Stress relaxation is defined as a gradual decrease in stress with time, under a constant strain. Compression molded tensile specimens will be strained in an Instron mechanical tester. The stress decay will be followed as a function of time. We predict an increase in stress relaxation rate due to plasticization. This study is very useful in predicting long term radiation effects. If the relaxation rate increases with irradiation dose or time, short term accelerated tests are more stringent than long term tests at lower dose rates. If the stress relaxation rate decreases with irradiation dose or time, the opposite conclusion is valid.

\section{g) TMA thermal Mechanical Analysis}

The build up of cooling stresses during injection molding is partly a result of the high coefficient of thermal expansion of plastics (14). The amount of contraction that takes place from the solidification temperature to the ambient temperature is determined by the CTE. We will characterize the CTE of our low $T_{3}$ thermoplastic scintillator as compared to that of commercial scintillator. A Dupont 2100 TMA will be used to characterize the coefficient of 
thermal expansion, CTE, of scintillator materials. The linear CTE, $B$, is defined as $B=1 / L$ ( $\partial \mathrm{L} \partial \mathrm{T}) \mathrm{p}$ where $\mathrm{L}$ is the sample length. This test will be conducted on scintillator that is used for injection molding.

This test will be performed on thin films $3 \mathrm{~mm} \times 15 \mathrm{~mm}$. The linear CTE is measured under a fixed load. The temperature is ramped from room temperature through the $T_{8}$ of the sample. The CTE is calculated from plots of simple gauge length versus temperanure.

\section{h) Bending Stress Analysis}

This test will be used to characterize the effect of bending stress on fiber integrity before and after irradiation. Wavelength shifting fibers will be deformed to a specific radius of curvature and fixed to the scintillator plates. This bending may cause damage to the fiber during processing or storage. In addition, elastic stresses present in the fiber during radiation may result in increased susceptibility to irradiation damage. Our plasticized fibers should offer the advantage of being highly flexible. Bending strengths will be measured by wrapping the fibers around dowels of different diameters. The bending stress, at failure, $s$, is calculated from:

$$
s=E r / \rho
$$

where $\mathrm{E}$ is the modulus obtained by the DMA as described above, $r$ is the radius of the fiber and $\rho$ is the radius of curvanure. Fibers will be irradiated while affixed to the dowels.

i) Mictoscopic examination

Compression and injection molded samples will be examined under the microscope before and after irradiation to observe any radiation induced crazying. 
j) Plasticizer Dye migration studies

It is important that plasticizers and dyes do not migrate from the polymer matrix. Migration, will be monitored by placing a doped plate in contact with thin films of pure polymer. $100 \mathrm{gm}$ weights will be placed on the samples. Samples will be incubated at room temperature, 35 and $45^{\circ} \mathrm{C}$. At one month intervals, the undoped films will be removed and FTIR spectra will be recorded on the film. The area under a selected peak due to the dye or plasticizer will be recorded. A series of films will be made with various concentrations of plasticizer or dye. FTIR spectra will be recorded on these samples to form a calibration curve. The concentration of plasticizer or dye which migrates from the plates will be calculated from this curve.

3. Budget for Task II Instrument Rental

4. Budget Justification for Task II

The Detector Development Group rents equipment from the University Progress Center in Alachua Florida.

5. Time Schedule for Task II

Aging Studies:

Onset of Testing 1 month

Completion of tests prior to radiation exposure 2 months

Radiation Exposure Time

1 day - 6 months

Final Testing Complete 1 week after removal 
from irradiator

Plasticizer Migration Study

3 months 
TASK III

Life Testing Materials From Other SSC Groups

\section{Table of Contents}

1. Scope of the Work

2. Physical Testing

3. Budget

4. Budget Justification

5. Time Schedule 
1. Scope of Work for Task III

This task involves evaluating materials made by other members of the SSC community and comparing the properties of these materials to the materials made by the Detector Development Group at $\mathrm{U}$. of $\mathrm{F}$.

2. Physical Testing for Task III

Physical testing is the same as in task II.

3. Budget for Task III

Equipment rental

4. Budget Justification for Task III

We will rent equipment for the proposed testing from the University Progress Center in Alachua.

5. Onset of testing

Completion of tests prior to radiation exposure

Radiation exposure times

Final testing complete
1 month

2 months

1 day - 6 months

1 week after removal

from irradiator 
TASK IV

STUDY OF NNECTION YOLDNG OF SCINTLLIATOR

TABLE OF CONTENTS

1. Properties of Injection Molder

2. Characterization of the Low Glass Transition Temperature Polystyrene System.

3. Develpment of Residual Stresses and Molecular Orientation on Injection molding of Scintillator Plate.

4a. Software Program to Minimize Residual Stresses in Injection Molded Parts

4b. Input Data Required by the Third Layer Analysis of C-YOLDo

5. Reducing Residual Stresses by Teflon Coating of Hold Surface

6. Injection Mold for Kaking Scintillator Plate

7. Assessment of Residual Stresses in Injection Molded Parts...

8. Prototype Plate Production.

9. References

10. Schedule and Budget Summary 


\section{Properties of lnjection Molder}

Since during the research and development stage, the available quantities of test material are often limitted. we choose an injection molding machine that is designed for this kind of prototyping and low volume production purpose.

An air-driven ram type injection molding machine from Morgar Industries. Inc. was ordered, which has 2 oz shot size capacity, 20 ton clamping force (toggle) and 13500 psi maximum injection pressure. This machine is also equipped with digital temperature controller, injection speed control, and preheat plate, which make the setting of the operating conditions more accurate.

2. Characterization of the Low Glass Transition Temperature Polystyrene System

Since the recovery process and apparently the resulting permanent damage of plastic scintillator from radiation is controlled by a first order polymer relaxation process ', we tried to speed up this relaxation process by reducing the glass transition temperature $\left(T_{h}\right)$ of the polymer. By adding a plasticizer, we are able to reduce the $\mathrm{T}_{\mathfrak{g}}$ of polystyrene from $\sim 100^{\circ} \mathrm{C}$ to $\sim 55^{\circ} \mathrm{C}$, and injection mold it at melt temperature of about $150^{\circ} \mathrm{C}$.

This low $T_{\mathbf{l}}$ polystyrene blend system exhibits such rapid relaxation that annealable color centers do not persist and it becomes optically radiation hard plasitc that is suitable for scintillator.

3. Develpment of Residual Stresses and Molecular Orientation on Injection molding of Scintillator Plate

Injection molding is a highly nonisothermal and anisotropic semi-batch process. The polymer experiences severe thermal-mechanical steps in this process. Polymer chains became permanently oriented in the flow direction during the filling 
stage in a frozen layer adjacent to the mold wall. Residual streuses in an injection molded part can be attributed to:

(1) the unrelaxed shear and normal stresses from the mold filling stage (residual flow stresses) due to frozen molecular orjentation.

(2) development of thermal (cooling) stresses in the cooling stage due to the viscoelastic behavior of the polymer when it passes through the glass transition temperature and the nonequilibrium density changes caused by the rapid, inhomogeneous cooling.

Typcal residual thermal stresses in a quenched polystyrene plastic plate are compressive on the surface, and tensile in the core region (Fig. 1). While the residual flow stresses are always tensile and increase from core to surface. Reducing melt temperature or injection speed will increase these residual flow stresses (Fig. 2a and $2 b)$. Hence, the resulting residual stresses in an injection molded polystyrene plastic plate will depend on these operating conditions and are shown as Fig. 3a and $3 b 2$. In order to minimize the residual stresses, we have to operate the injection molding machine at lower melt temperature and injection speed.

Existence of residual stresses will cause crazing with aging and deteriorate the mechanical properties and optical transparency of the scintillator plate. Besides, the residual stresses will also increase the sensitivity of the plate to radiation damage. Molecular orientation and residual stresses also cause optical anisotropy and nonuniformity of refractive index which also reduce the efficiency of the plate.

The overall effects of molecular orjentation and residual stresses can be estimated by measuring the birefringence of the sample.

4a. Software Program to Minimize Residual Stresses in Injection Molded Parts 


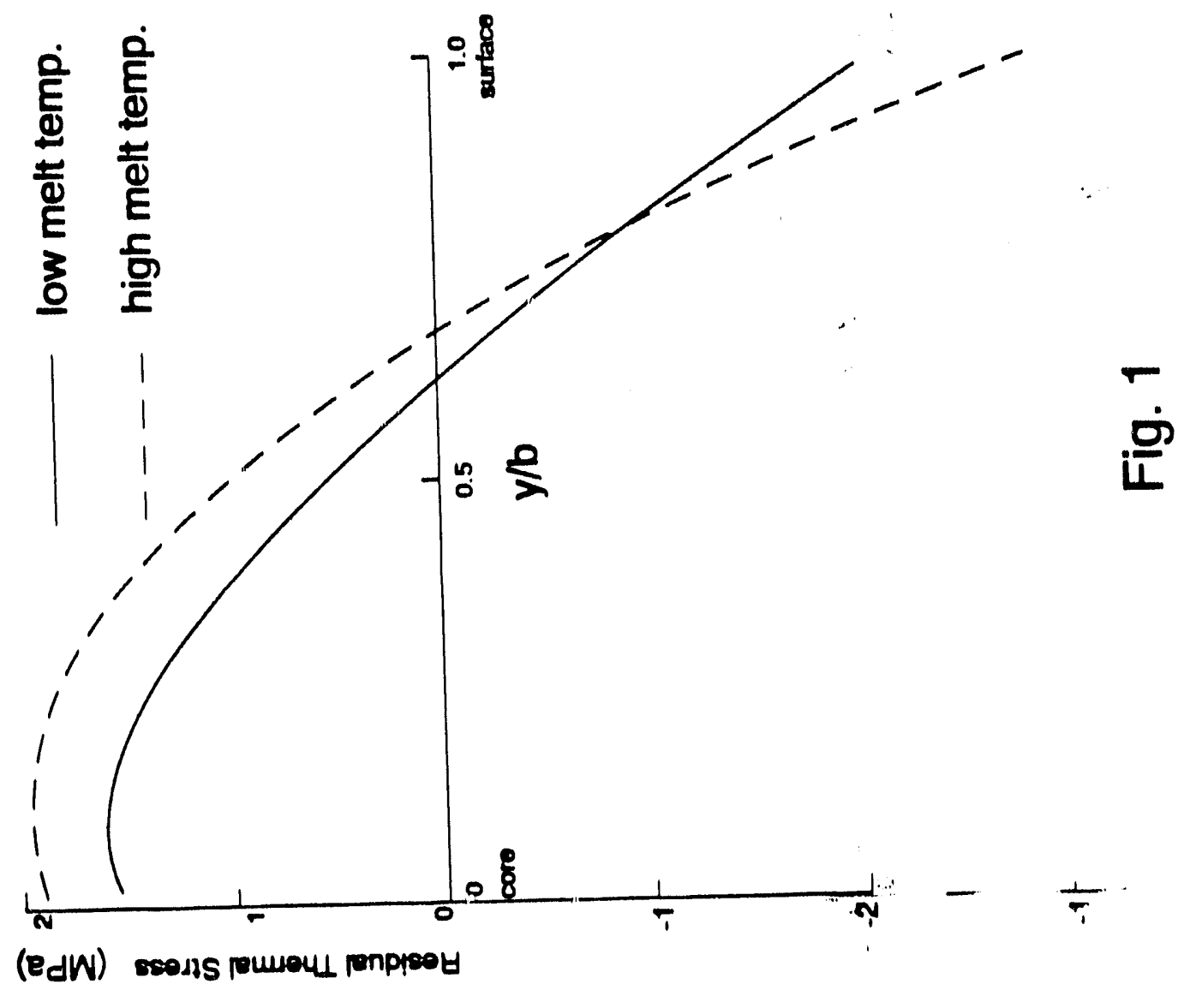




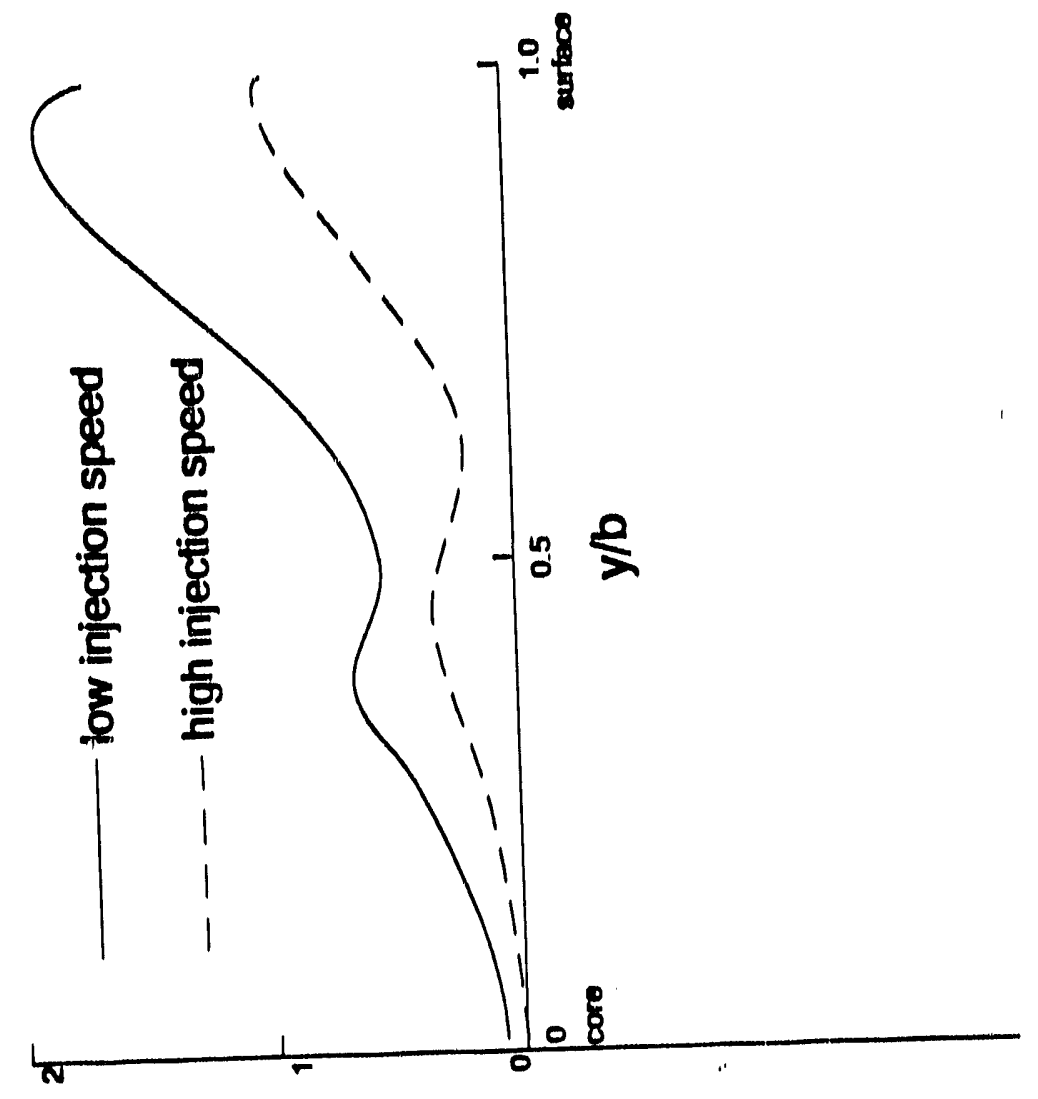

ลี

(EdW) ssens MOld renplset

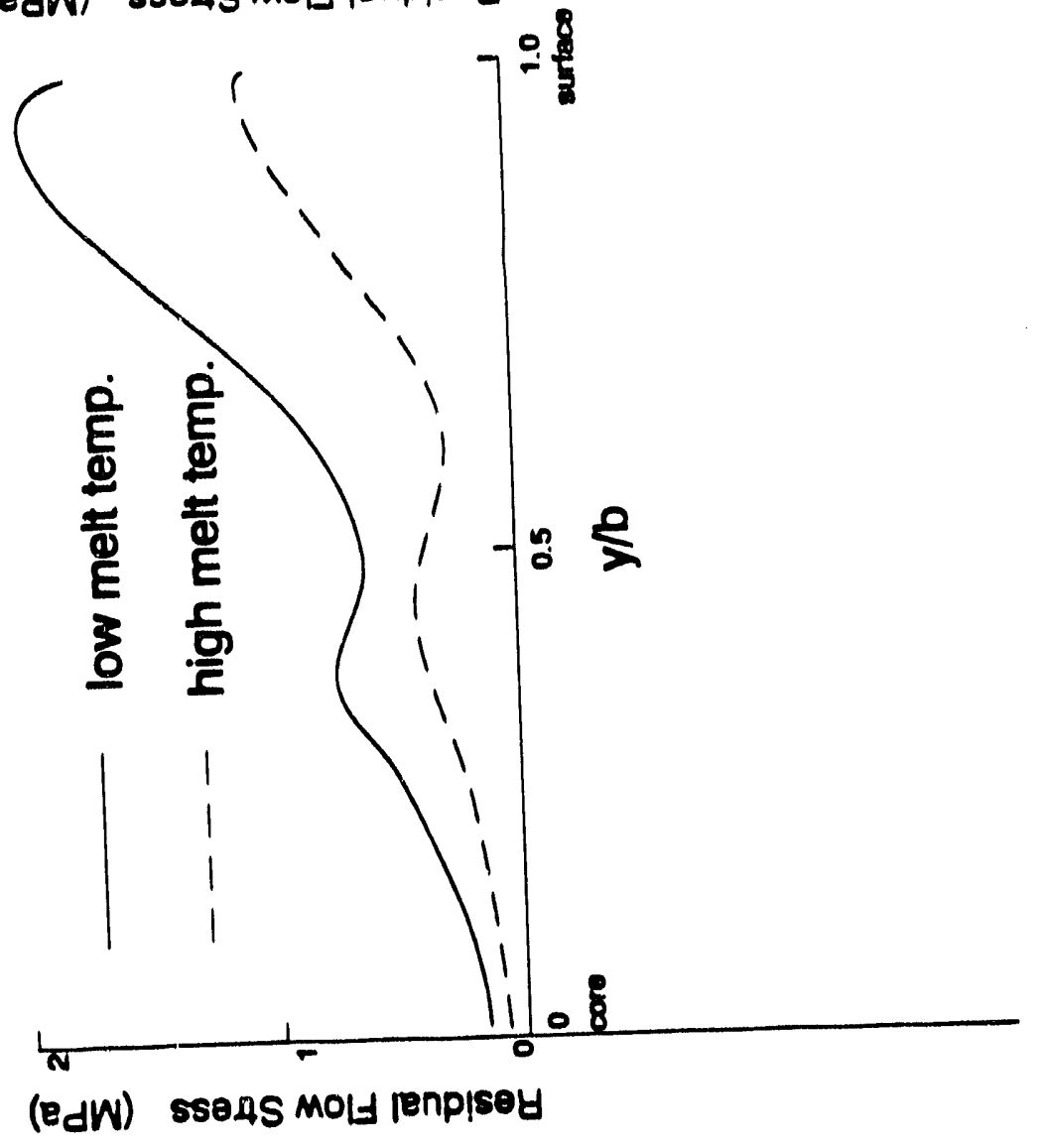

ชิ

ix 

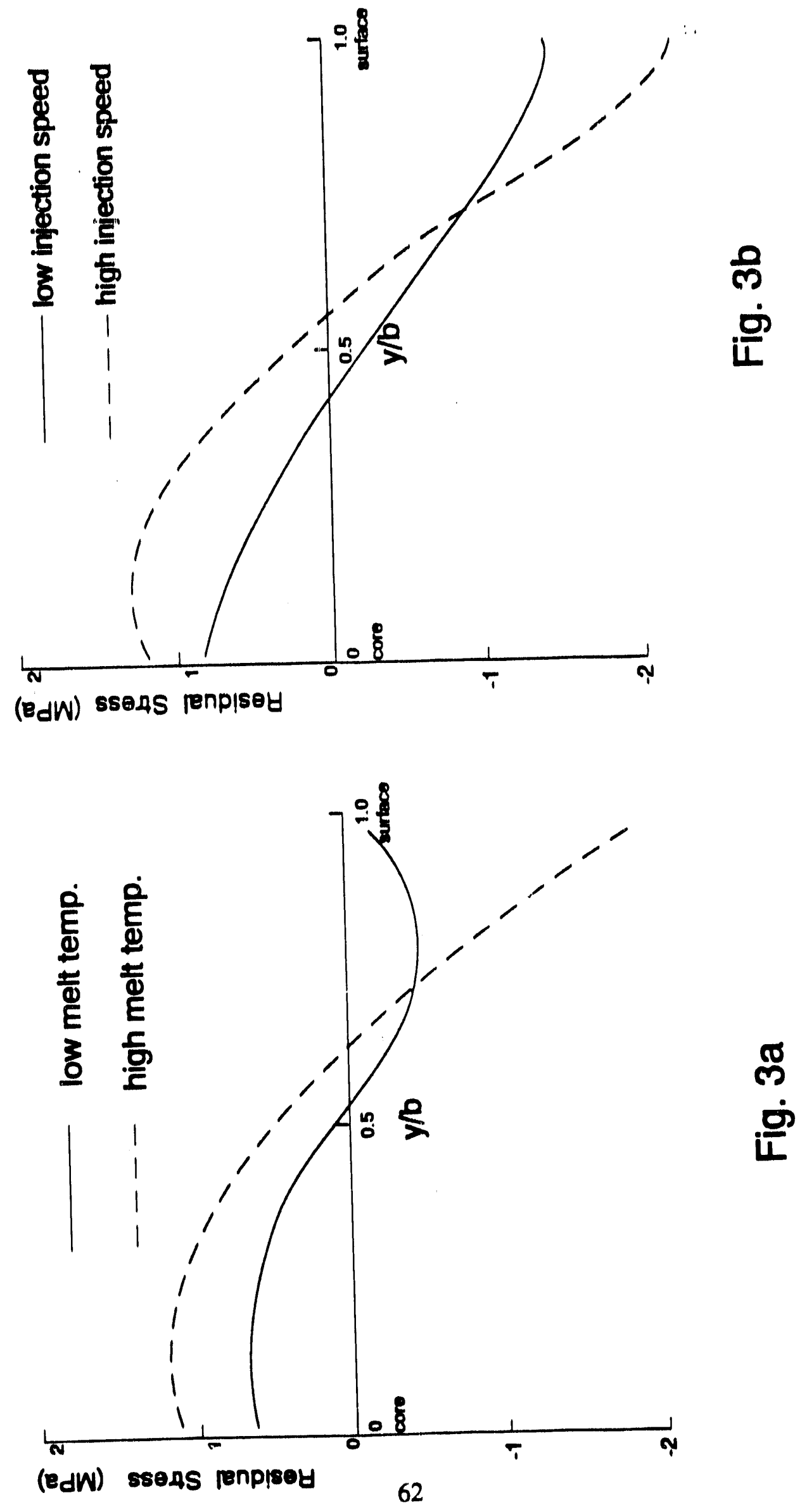
In order to minimize the residual stresses developed in the molded parts and any potential warpage, we have to build a mold, select a proper gate location (or locations), and operate the injection molding machine at conditions that will introduce minimum amount of frozen-in stresses during the mold filling stage. We must also provide uniform cooling at a rate slow enough to avoid thermal stresses from being frozen in, yet fast enough to achieve reasonable productivity.

The C-MOLDD software package developed by AC Technology, Inc. uses an iterative process which integrates all aspects of production, from part and mold design to the selection of materials and setting up the process conditions. By using its three-layer integrated CAE approach (Fig. 4) one can evaluate the interaction of these variables with the computer before mold trials are instituted.

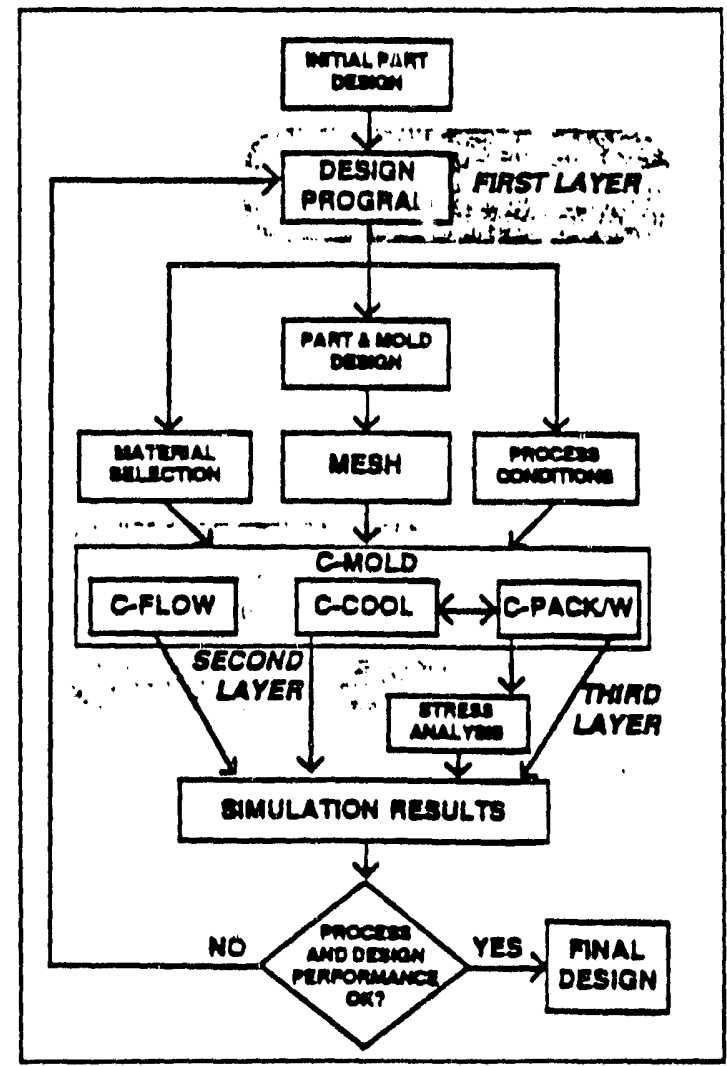

Figure 4 Overall structure of the three-layer CAE approach. 
In the third layer of this approach (including C-PACK/Wo and C-COOL), the program performs three-dirnensional unified mold filling/postfilling/residual stress analyses concurrently with a mold cooling analysis (Fig. 5). This analysis can handle material properties as functions of temperature, pressure and/or shear rate. Stresses development in the vitrifying plastic part are calculated through the simultaneous application of non-homogeneous cooling and packing pressure.

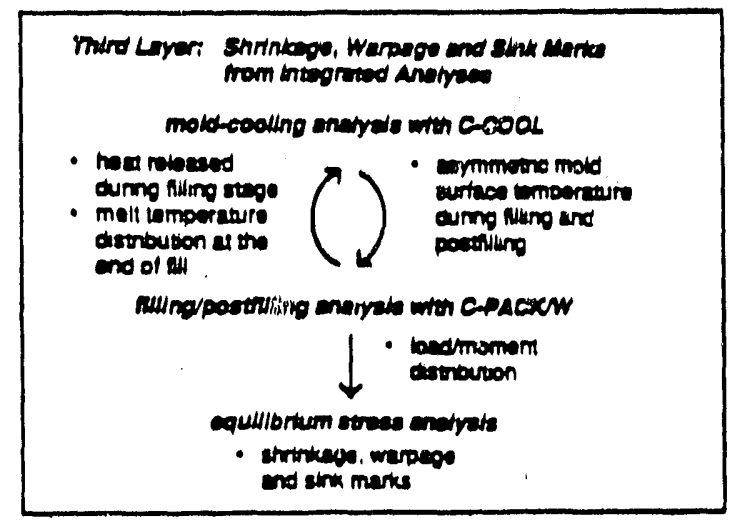

Figure 5 Functionalities and data communication between different modules in the third layer.

4b. Input Data Required by the Third Layer Analysis of C-MOLDo

The third layer analysis requires the polymer material properties of $\mathrm{p}-\mathrm{v}-\mathrm{T}$, shear viscosity, specific heat and thermal conductivity, as well as moduli and expansion/shrinkage ratio. These data are available for general purpose polystyrene in the data bank of AC Technology, Inc.. We plan to measure these material properties for the final low $T_{l}$ plastic chosen for use at the SSC. Nold and coolant material * properties are also required as input information.

Users have to specify process conditions, such as initial melt temperature. maximum injection pressure, injection ram speed profile, fill and holding time, fill-topack transition, coolant temperature and flow rate (pressure drop). 


\section{Reducing Residual Stresses by Teflon Coating of Mold Surface}

Residual stresses in injection molded parts can be further reduced by applying a passive low thermal inertia insulation layer on the cavity surface. This layer can increase the cavity surface temperature during mold filling thus preventing the polymer melt from freezing during filling, and allowing the flow-induced stresses to relax after filling. Reduction of birefringence by $35 \%$ and increase of cooling time by less than $20 \%$ were achieved by injection molding of polystyrene in a Teflon coated mold 3 .

\section{Injection Mold for Making Scintillator Plate}

A universal 5" $\times 8 " 3$-Plate mold base by DME. Inc. supplies the knock-out device and housing for our core and cavity plates. The core plate (Fig. 6) with a w-shape protrusion is mounted to the upper platen and stays stationary. The cavity plate (Fig. 7) is mounted on top of the ejector housing and will move downward when the mold is open. A $10 \times 10 \times 0.25 \mathrm{~cm}^{3}$ plate can be molded with a $w$-shape $1 \mathrm{~mm}$ wide groove on it. The scintillating optical fiber can be mounted into this groove.

\section{Assessment of Residual Stresses in lnjection Molded Parts}

The most suitable means for experimentally determining the gapwise residual stress distribution is the layer-removal method 4 . In this method. thin layers of uniform thickness are removed by a milling machine from one surface of a rectangular specimen thus changing the equilibrium of the original residual stress distribution. In order to reestablish equilibrium, the specimen will warp to the shape of a circular arc. The original residual stress distribution can be calculated by measuring the resultant curvature (by microscope) as a function of depth of material removed using the following equation. 


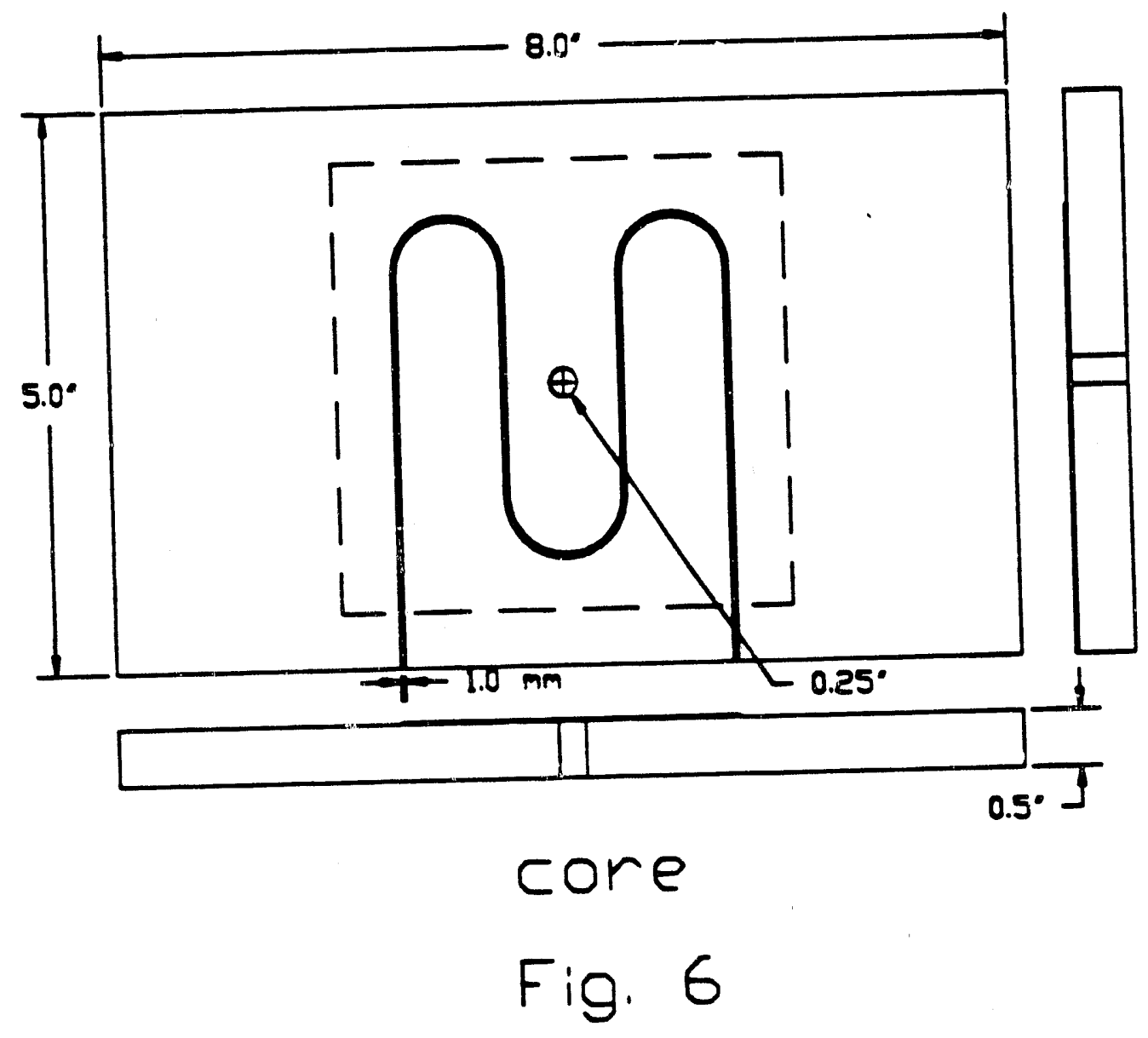




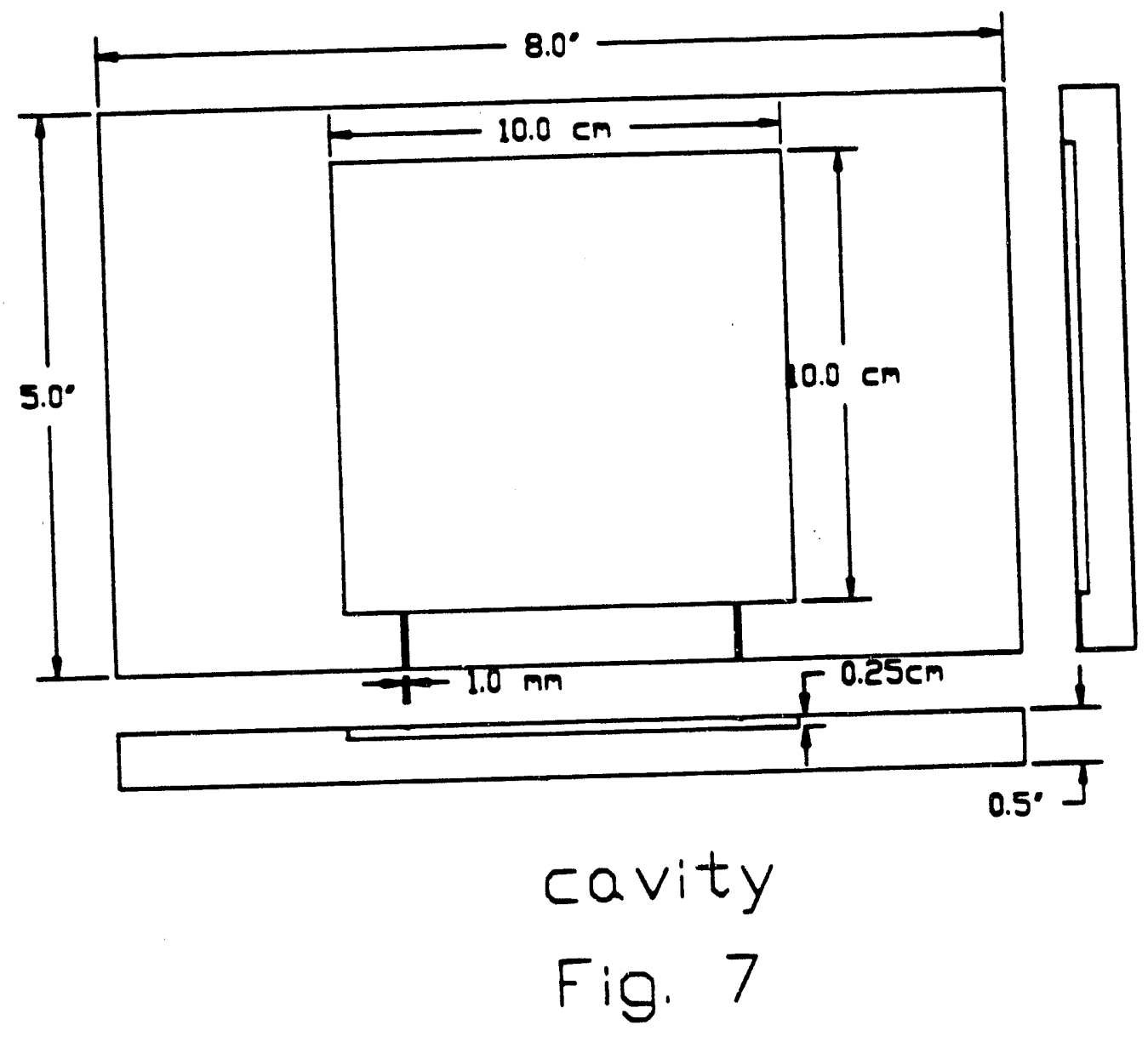




$$
\begin{aligned}
\sigma_{x}\left(y_{1}\right)= & \frac{-E}{6\left(1-v^{2}\right)}\left\{\left(b+y_{1}\right)^{2}\right. \\
& \left.b \quad \frac{d \rho_{x}\left(y_{1}\right)}{d y_{1}}+\frac{v d \rho_{2}\left(y_{1}\right)}{d y_{1}}\right]+4\left(b+y_{1}\right)\left[\rho_{x}\left(y_{1}\right)+v d \rho_{z}\left(y_{1}\right)\right] \\
& \left.-2 \int_{y_{1}}\left[\rho_{x}(y)+v d \rho_{2}(y)\right] d y\right\}
\end{aligned}
$$

where $\sigma_{x}$ is the longitudinal stress, $y= \pm b$ are the initial upper and lower surfaces of the specimen, $y=y_{1}$ is the newly formed surface after each layer removed, $E$ is the elastic modulus, $v$ is the poisson's ratio, and $\rho_{x}$ and $\rho_{z}$ are the measured longitudinal and transverse curvature respectively. $\sigma_{2}\left(y_{1}\right)$ can be similarly calculated by exchanging $x$ and $z$ in the above equation.

There is another indirect method of measuring these residual stresses. The birefringence of the injection molded part contains contributions from residual stresses and frozen-in molecular orientation. By thin slicing the sample (longitudinal length of sample must be less than $1 / 5$ of the gaprise thickness) to free these residual stresses in the longitudinal direction, the measured birefringence contains only the contribution from the molecular orientation. The difference of the birefringence between the original sample and the thin sliced sample gives us a measurement of the residual stress inside the injection molded sample.

\section{Specific Nork Program}

\section{a) Polystyrene Plate}

The above set of computer simulations and experimental measurements of residual stresses will be performed using polystyrene as the plastic matrix. He will assess the reliability of the computer simulations to predict the measured residna] stressed in the plate. THis procedure is shown schematically in Fig. 8.

b) Lor T, Polystyrene Plate

As the choice of specific form low $\mathrm{T}_{\mathrm{a}}$ polystyrene plastic becomes more focussed, we will perform the necessary set of measurements on that plastic as input data for the simulation of residual stresses. We will use the program to search for 
the optimum set of injection molding conditions to minimize stresses. Measurement of these stresses will be performed to confirm that the plates have the desired properties.

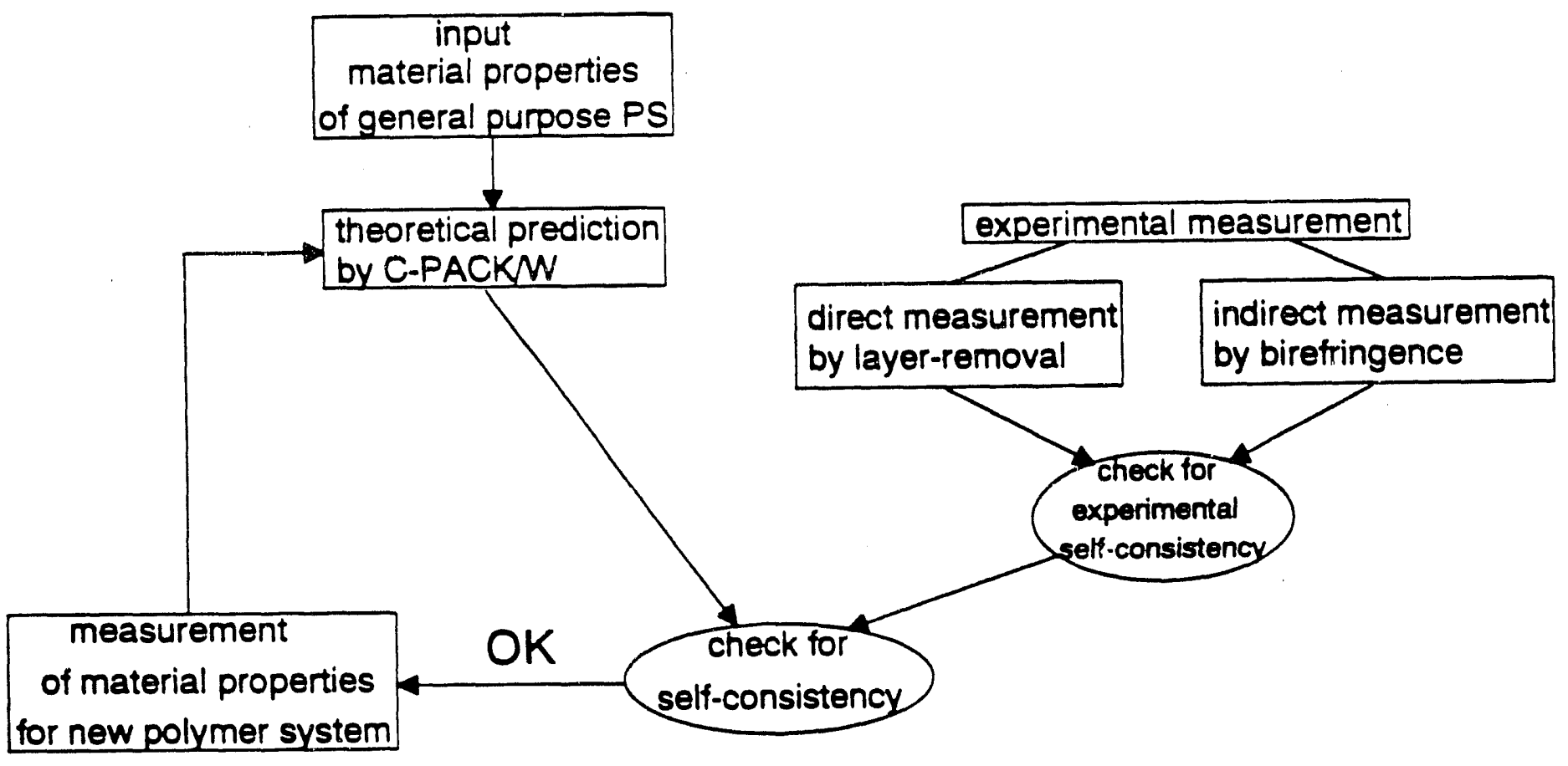

Fig. 8 
8. Prototype Plate Production

The objective of this work is to produce scintillating plates which will be distributed for testing purposes to members of the SDC collaboration.

\section{References}

1. Harmon. J. P. and J. K. Walker. Symposium on Detector Research for the Superconducting Super Collider (1990).

2. Isayev. A.I. and D. L. Crouthamel, Polym.-Plast. Tech. Eng. 22(2). 177 (1984).

3. Liou. M. and N. Suh. SPE Technical Papers 34, 324 (1988).

4. Treuting, R. G. and W T. Read. Jr., Appl. Phys. 22. 130 (1951).

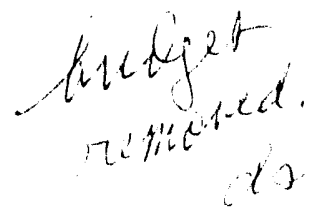


IV. Facilities Available:

1. Material Production - We have all of the equipment necessary to polymerize scintillator, wave length shifter and cladding polymers, ie) balance, ovens, water bath, fume hood.

2. Materials testing - The Detector Development Group has access to the following equipment:

Hewlett Packard HP 8451A Spectrophotometer

Hewlett Packard 1046A Programmable Fluoresce Detector

$600 \mathrm{Ci}^{60} \mathrm{Co}$ Gamma Radiation Source

*Gel Permeation Chromatograph

*Thermal Mechanical Analysis Unit

*Fourier Transform Infrared Spectrometer

*Dynamic Mechanical Analyzer

*Light Microscope

*These instruments are rented from the Progress Center.

3. Injection Molding - The Detector Development Group at U. of F. has purchased an air-driven ram injection molder from Morgan Industries. We have access to a microscope unit for measuring birerigence and access to a machine shop for layer removal procedures. 


\section{Personnel}

Dr. J. K. Walker, high energy physicist, is the principle investigator on this proposal. He has 15 years experience at Fermi National Laboratory leading major projects. He was a consultant to the Pilot Chemical Company, the largest producer of scintillating plastic in the United States.

Dr. Julie Harmon, a co-principal investigator, is an Associate Research Scientist in the Physics Department at the University of Florida. She has previously worked as a Research Scientist with Eastman Kodak. She has extensive experience in the area of polymer synthesis and processing (extrusion, roll mill compounding). She holds patents in the area of dyepolymer interactions.

Dr. C. M. Hsuing is an Assistant in Physics at the University of Florida. He was a Post Doctoral Associate in polymer engineering at the University of Akron where he managed a laboratory. He is an expert in the area of injection molding and melt spinning. He has done extensive studies on computer simulation of the effect of injection molding parameters on orientation and structure development in molded ports. 

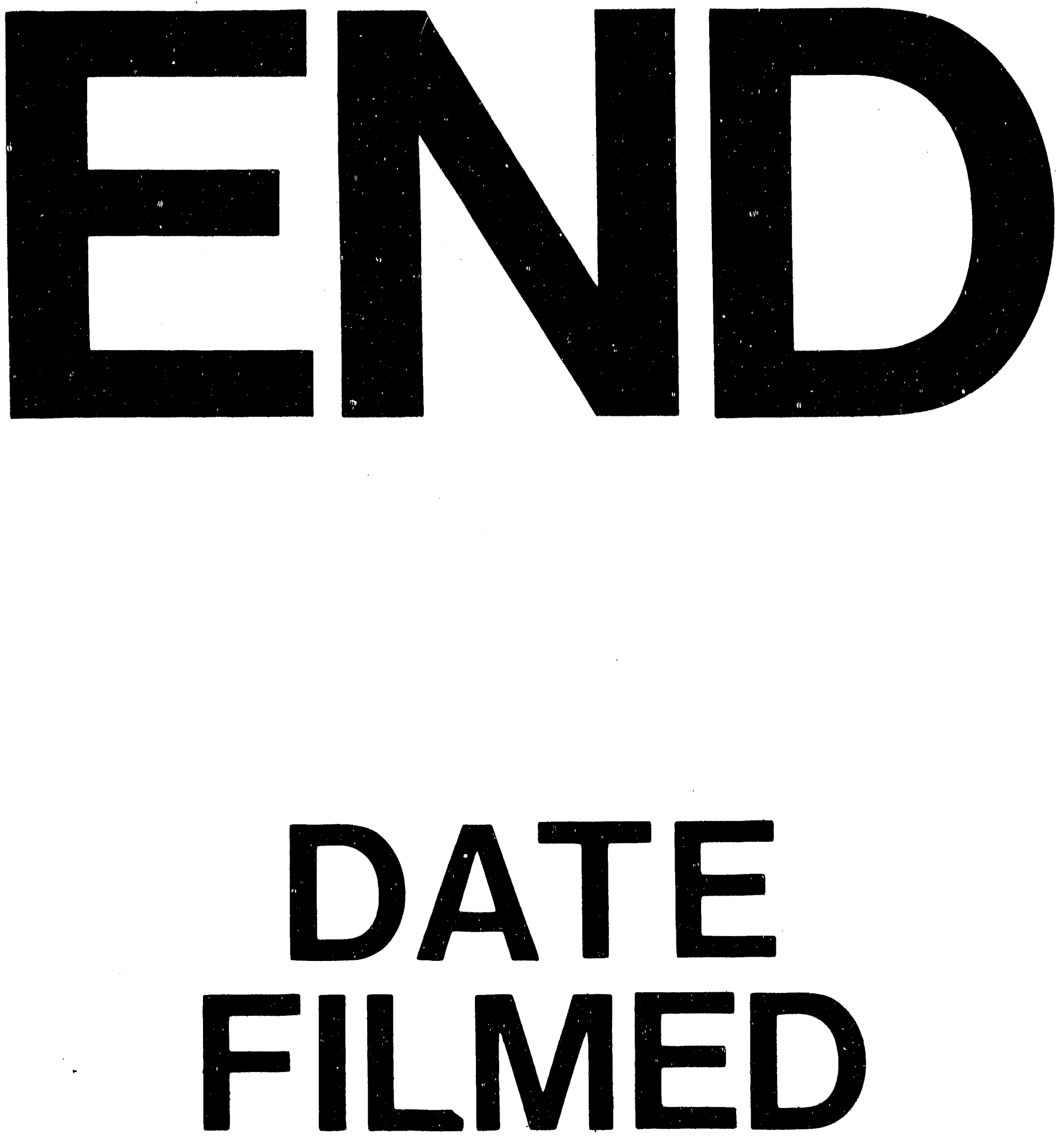

I

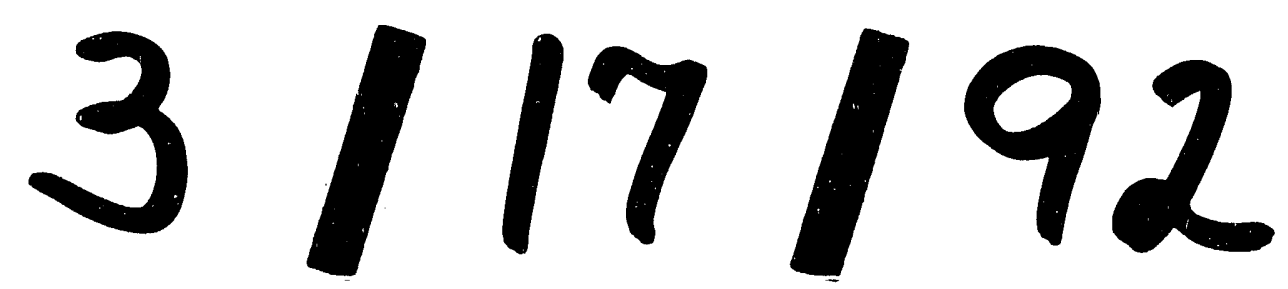


- 